\title{
A DG GUIDE TO VOEVODSKY'S MOTIVES
}

\author{
A. Beilinson and V. Vologodsky \\ The University of Chicago
}

To Joseph Bernstein with love and gratitude.

Let $\mathcal{V} a r$ be the category of complex algebraic varieties, Top that of nice topological spaces, $D_{a b}$ be the derived category of finite complexes of finitely generated abelian groups. One has tensor functors $\mathcal{V} a r \rightarrow T o p \rightarrow D_{a b}$, the first assigns to a variety its space equipped with the classical topology, the second one is the singular chain complex functor (the tensor structure for the first two categories is given by the direct product). The basic objective of the motive theory is to fill in a commutative square

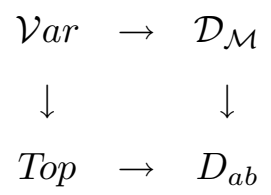

where $D_{\mathcal{M}}$ - the category of motives - is a rigid tensor triangulated category defined, together with the upper horizontal arrow, in a purely geometric way (so that the base field $\mathbb{C}$ can be replaced by any field), and the right vertical arrow is a tensor triangulated functor (which absorbs all the transcendence of the singular chains).

The known constructions (due to Hanamura, Levine, and Voevodsky) proceed by first embedding $\mathcal{V}$ ar into a larger DG category, and then define $D_{\mathcal{M}}$ as its appropriate quotient. Voevodsky's generators and relations are especially neat. A rough idea: consider the localization of the DG category freely generated by the category of topological manifolds modulo the relations that kill the complexes of types $\mathbb{Z}[\Delta \times X] \rightarrow \mathbb{Z}[X]$ and $\mathbb{Z}[U \cap V] \rightarrow \mathbb{Z}[U] \oplus \mathbb{Z}[V] \rightarrow \mathbb{Z}[X]$ (here $\Delta$ is an interval, $\{U, V\}$ is an open covering of $X)$; the singular chains functor yields then an equivalence between this toy category of "topological motives" and $D_{a b}$. To define $\mathcal{D}_{\mathcal{M}}$, one formally imitates this construction in the algebro-geometric setting with an important modification: mere combinations of true algebraic maps should be replaced from scratch by a larger group of finite correspondences, i.e., multi-valued maps (which is irrelevant in the topological setting).

What follows is a concise exposition of Voevodsky's theory that covers principal points of [Vo1-3] and [MVW] with the notable exception of comparison results (relating the motivic cohomology with Bloch's higher Chow groups and Milnor's Kgroups, and the étale localized motives with finite coefficients with Galois modules, see $[\mathrm{MVW}] 19.1,5.1$, [Vo2] 3.3.3). The more advanced subjects of $\mathbb{A}^{1}$-homotopy theory, the proof of the Milnor-Bloch-Kato conjecture, and the array of motivic dreams, are not touched.

We take the time to spell out the basic constructions on the DG category level ([Vo2] and [MVW] consider mere triangulated category structure). For the present material, this has the limited advantage of making formulas like (4.4.1), (4.4.2) 
possible, but seems to be necessary for some future developments (such as understanding of the motivic descent). The required generalities are recalled in (the lengthy) $\S 1$; we use concrete Keller's construction of homotopy DG quotients (see $[\mathrm{K}]$ and $[\mathrm{Dr}] \S 4)$ which suffices for immediate purposes. In truth, unfettered DG functoriality requires better understanding of the world of DG categories (e.g. to be able to determine a DG category à la Yoneda by the homotopy DG 2-functor it represents). Hopefully, the necessary material can be found in Lurie's treatise [L1], [L2].

We are grateful to Volodya Drinfeld and Andrey Suslin for teaching us, respectively, DG categories and motives. Our deep thanks due to Volodya Drinfeld, Dennis Gaitsgory, Madhav Nori, Chuck Weibel, and the referee for their truly generous help in turning a raw draft into an article. The research was partially supported by NSF grant DMS-0401164.

\section{$\S 1$. Homological algebra recollections}

The basic reference for triangulated categories is [Ve]; for the DG story it is [Dr]. The format of 1.3 helps to formulate Voevodsky's main technical result (see 4.4).

1.1. Below $R$ is a fixed commutative ring, say, $R=\mathbb{Z}$. We play with $R$ categories. Let $A$ be one. For a full subcategory $I \subset A$ its right orthogonal complement is $I^{\perp}:=\{M \in A: \operatorname{Hom}(N, M)=0 \forall N \in I\}$; the left orthogonal complement ${ }^{\perp} I$ is defined in the dual way. We say that $A$ is cocomplete if it is closed under direct sums of arbitrary cardinality. For such an $A$, an object $M \in A$ is said to be compact if for every set of objects $\left\{N_{\alpha}\right\}$ one has $\oplus \operatorname{Hom}\left(M, N_{\alpha}\right) \stackrel{\sim}{\rightarrow} \operatorname{Hom}\left(M, \oplus N_{\alpha}\right)$.

We denote by $A^{\mathrm{op}}$ the dual category; $M \mapsto M^{\mathrm{op}}$ is the contravariant "identity" functor $A \rightarrow A^{\mathrm{op}}$. $A^{\kappa}$ is the idempotent completion (a.k.a. Karoubianization) of $A$. For $A$ essentially small, $A$-mod is the category of $A$-modules, i.e., $R$-linear functors $A \rightarrow R$-mod (the category of $R$-modules); this is a cocomplete abelian $R$-category.

If $D$ is a triangulated category, then such is $D^{\kappa}$ (see [BS]).

Remarks. (i) According to [Th], arbitrary triangulated categories can be described in terms of Karoubian ones as follows. Let $D$ be a small Karoubian triangulated category. For a subgroup $H$ of the Grothendieck group $K(D)$ let $D_{H} \subset D$ be the subcategory of objects whose classes lie in $H$. Then the map $H \mapsto D_{H}$ is a bijection between the set of subgroups of $K(D)$ and that of strictly full triangulated subcategories $C \subset D$ such that $C^{\kappa}=D$.

(ii) In many situations it is easy to check if a given triangulated category is Karoubian. E.g., in [BS] it is shown that the bounded derived category of a Karoubian exact category is Karoubian; for other examples, see 1.4.1 and 1.5.6.

1.2. Admissible subcategories. Let $D$ be a triangulated ( $R$-) category, $I \subset$ $D$ be a strictly full triangulated subcategory. $I$ is said to be thick if any direct summand of an object of $I$ lies in $I$.

For $M \in D$ a right $I$-localization triangle for $M$ is an exact triangle $M_{I} \stackrel{\mu}{\rightarrow} M \stackrel{\nu}{\rightarrow}$ $M_{I^{\perp}}$ with $M_{I} \in I$ and $M_{I^{\perp}} \in I^{\perp}$. If such a triangle exists, then it is unique (up to a uniquely defined isomorphism). If it exists for every $M \in D$, then $I$ is said to be right admissible. This amounts to either of the following properties: 
(i) The embedding functor $i_{*}: I \rightarrow D$ admits a right adjoint $i^{!}: D \rightarrow I$.

(ii) $I$ is a thick subcategory of $D$, the Verdier quotient $D / I$ is well defined, and the projection functor $j^{*}: D \rightarrow D / I$ admits a right adjoint $j_{*}: D / I \rightarrow D$.

If this happens, then $M_{I}=i_{*} i^{!} M$ and $M_{I^{\perp}}=j_{*} j^{*} M$. Also $I^{\perp}$ is a full triangulated subcategory of $D, I \stackrel{\sim}{\rightarrow}{ }^{\perp}\left(I^{\perp}\right)$, the projection $I^{\perp} \rightarrow D / I$ is an equivalence of categories, and $j_{*}$ is the composition of the inverse equivalence and the embedding $I^{\perp} \hookrightarrow D$. The endofunctor $\mathcal{C}_{I}=j_{*} j^{*}$ of $D, \mathcal{C}_{I}(M)=M_{I^{\perp}}$, equipped with the morphism of functors $\nu=\nu_{I}: \operatorname{Id}_{D} \rightarrow \mathcal{C}_{I}$, is called the (right) I-localization. Notice that the pair $\left(\mathcal{C}_{I}, \nu_{I}\right)$ admits no non-trivial automorphisms

Example. Let $X$ be a topological space, $i: Y \hookrightarrow X$ its closed subspace, $j$ : $U \hookrightarrow X$ the complement to $Y$. Let $D$ be the derived category of sheaves of abelian groups on $X$, and $I$ that on $Y$. Then $i_{*}: I \rightarrow D$ is a fully faithful functor whose essential image is right admissible, and $j^{*}$ identifies $D / I$ with the derived category of sheaves on $U$. The same is true in the $\mathcal{O}$ - and $\mathcal{D}$-module setting of algebraic geometry.

There is a dual notion of left-admissible subcategory; $I \leftrightarrow I^{\perp}$ is a 1 -1 correspondence between the "sets" of right- and and left-admissible subcategories of $D$. The duality $D \mapsto D^{\text {op }}$ interchanges left- and right-admissible subcategories. The functors $\left(j_{*}, j^{*}, i_{*}, i^{!}\right)$for $I^{\perp \text { op }} \subset D^{\text {op }}$ are the same as functors $\left(i_{*}, i^{!}, j_{*}, j^{*}\right)$ for $I \subset D$.

Suppose we have a t-structure on $D$ (see $[\mathrm{BBD}] 1.3$ ), and let $I \subset D$ be a rightadmissible subcategory. The next lemma is due to D. Gaitsgory:

Lemma. (i) $\mathcal{C}_{I}$ is left exact if and only if $D / I$ admits a $t$-structure such that $j^{*}$ is exact. The latter t-structure is unique.

(ii) $\mathcal{C}_{I}$ is right exact if and only if $D / I$ admits a t-structure such that $j_{*}$ is t-exact. The latter t-structure is unique.

Exercise. Condition (i) for $I \subset D$ implies (ii) for $I^{\perp \text { op }} \subset D^{\text {op }}$ (and the opposite t-structure).

1.3. Compatibility. Two right-admissible subcategories $I_{1}, I_{2}$ of $D$ are said to be compatible if every $M \in D$ can be fitted into a commutative diagram

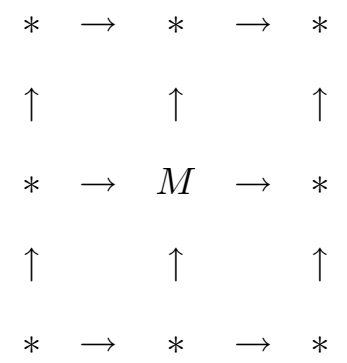

with exact rows and columns such that the bottom row lies in $\mathcal{I}_{1}$, the top row in $I_{1}^{\perp}$, the left column in $I_{2}$, and the right column in $I_{2}^{\perp}$. In other words, the columns are (right) $I_{1}$-localization triangles, and the rows are $I_{2}$-localization ones. 
Lemma. Compatibility of $I_{1}, I_{2}$ amounts to either of the next properties:

(i) For every $M \in D$ there exist morphisms $M_{0} \rightarrow M_{1} \rightarrow M$ such that $M_{0} \in$ $I_{1} \cap I_{2}$, Cone $\left(M_{0} \rightarrow M_{1}\right) \in\left(I_{1} \cap I_{2}^{\perp}\right) \times\left(I_{1}^{\perp} \cap I_{2}\right) \subset D$, Cone $\left(M_{1} \rightarrow M\right) \in I_{1}^{\perp} \cap I_{2}^{\perp}$.

(ii) The $I_{2}$-localization $\mathcal{C}_{2}=j_{2 *} j_{2}^{*}$ preserves both $I_{1}$ and $I_{1}^{\perp}$.

(ii) The endofunctor $i_{2 *} i_{2}^{!}$preserves both $I_{1}$ and $I_{1}^{\perp}$.

(iii) $\mathcal{C}_{2}$ preserves $I_{1}$, and $\mathcal{C}_{1}$ preserves $I_{2}$.

$\left(\right.$ iii) ${ }^{\prime} i_{2 *} i_{2}^{!}$preserves $I_{1}$, and $i_{1 *} i_{1}^{!}$preserves $I_{2}$.

(iv) The endofunctors $\mathcal{C}_{1} \mathcal{C}_{2}$ and $\mathcal{C}_{2} \mathcal{C}_{1}$ are isomorphic.

Let $I_{12}$ be the triangulated subcategory of $D$ strongly generated by $I_{1}$ and $I_{2}$ (see 1.4.2), so $I_{12}^{\perp}=I_{1}^{\perp} \cap I_{2}^{\perp}$. If $I_{1}, I_{2}$ are compatible, then $I_{12}$ is right admissible, the $I_{12}$-localization functor $\mathcal{C}_{12}$ is canonically isomorphic to $\mathcal{C}_{1} \mathcal{C}_{2}$ and $\mathcal{C}_{2} \mathcal{C}_{1},{ }^{1}$ and the projections $I_{1}^{\perp} / I_{1}^{\perp} \cap I_{2} \leftarrow I_{1}^{\perp} \cap I_{2}^{\perp} \rightarrow I_{2}^{\perp} / I_{2}^{\perp} \cap I_{1}$ are equivalences.

Examples. (i) In the example of 1.2, the categories $I=I_{Y}$ for various closed $Y$ 's are pairwise compatible. (ii) If $D$ is the derived category of $\mathcal{D}$-modules on the line $\mathbb{A}^{1}$, then the subcategory of $\mathcal{D}$-modules supported at a point and its Fourier transform are not compatible.

1.4. Cocomplete vs. small \& Karoubian. The results of 1.4.1, 1.4.2 are due to A. Neeman [N], those of 1.4.3 to V. Drinfeld.

1.4.1. Let $D$ be a triangulated category. For a diagram $M_{0} \stackrel{i_{0}}{\rightarrow} M_{1} \stackrel{i_{1}}{\rightarrow} M_{2} \stackrel{i_{2}}{\rightarrow} \ldots$ in $D$ we set $\operatorname{hocolim}\left(M_{a}, i_{a}\right):=\mathcal{C}$ one $\left(\oplus M_{a} \stackrel{\nu}{\rightarrow} \oplus M_{a}\right)$ where $\nu\left(m_{a}\right):=m_{a}-i_{a}\left(m_{a}\right)$. Notice that, as cones do, the object hocolim $\left(M_{a}, i_{a}\right)$ is defined up to a non-canonical isomorphism. There are canonical morphisms $\mu_{a}: M_{a} \rightarrow \operatorname{hocolim}\left(M_{a}, i_{a}\right)$ such that $\mu_{a+1} i_{a}=\mu_{a}$; for every collection of morphisms $\phi_{a}: M_{a} \rightarrow N$ such that $\phi_{a+1} i_{a}=\phi_{a}$ there exists a morphism $\phi: \operatorname{hocolim}\left(M_{a}, i_{a}\right) \rightarrow N$ such that $\phi \mu_{a}=\phi_{a}$.

Lemma. If $D$ is closed under countable direct sums, then $D$ is Karoubian.

Proof. The image of an idempotent $\pi \in \operatorname{End} M$ is $M_{\pi}:=\operatorname{hocolim}\left(M_{a}, i_{a}\right)$, where $M_{a} \equiv M, i_{a} \equiv \pi$, equipped with the structure maps $M_{\pi} \rightarrow M$ induced from $\phi_{a}=\pi$ and $M \rightarrow M_{\pi}$ equal to $\mu_{0}$.

1.4.2. For a class $S$ of objects of $D$ the triangulated subcategory strongly generated by $S$ is the smallest strictly full triangulated subcategory $\langle S\rangle$ that contains $S$. If $D$ is cocomplete, then the triangulated subcategory generated by $S$ is the smallest strictly full triangulated subcategory of $D$ that contains $S$ and is closed under arbitrary direct sums.

If $I \subset D$ is a thick subcategory, then we set $D /{ }^{\kappa} I:=(D / I)^{\kappa}=\left(D^{\kappa} / I^{\kappa}\right)^{\kappa}$.

If $D$ is a cocomplete triangulated category, then $D^{\text {perf }} \subset D$ denotes the full subcategory of compact, a.k.a. perfect, objects. This is a Karoubian subcategory.

Proposition. Suppose $D$ is cocomplete and let $S \subset D$ be a set of perfect objects.

\footnotetext{
${ }^{1}$ Precisely, $\left(\mathcal{C}_{12}, \nu_{12}\right)=\left(\mathcal{C}_{1} \mathcal{C}_{2}, \nu_{1} \nu_{2}\right)=\left(\mathcal{C}_{2} \mathcal{C}_{1}, \nu_{2} \nu_{1}\right)$
} 
Set $I^{o}:=\langle S\rangle$, and let $I$ be the triangulated subcategory generated by $S$.

(i) $I$ is right admissible, and $I^{\text {perf }}=\left(I^{o}\right)^{\kappa}$.

(ii) The category $D / I$ is cocomplete, the adjoint functors $\left(j^{*}, j_{*}\right): D \leftrightarrows D / I$, $\left(i_{*}, i^{!}\right): I \leftrightarrows D$ commute with arbitrary direct sums, and $j^{*}\left(D^{\text {perf }}\right) \subset(D / I)^{\text {perf }}$

(iii) If $D$ is generated by a set of perfect objects, then $D^{\text {perf }} /{ }^{\kappa} I^{\text {perf }} \stackrel{\sim}{\rightarrow}(D / I)^{\text {perf }}$.

(iv) Every object of I can be represented as hocolim $\left(M_{a}, i_{a}\right)$ (see 1.4.1) where $M_{0}$ and each $\mathcal{C}$ one $\left(i_{a}\right)$ are direct sums of translations of objects from $S$.

Proof. We can assume that $S$ is closed with respect to translations: $S[ \pm 1]=S$. Then $S^{\perp}=I^{\perp}$.

(a) For $M \in D$ let us construct a right $I$-localization triangle with $M_{I}$ as in (iv).

Suppose we have a diagram $\left(M_{a}, i_{a}\right)$, as in 1.4.1, in $I$ and morphisms $\phi_{a}: M_{a} \rightarrow$ $M$ such that $\phi_{a+1} i_{a}=\phi_{a}$ with the next property: If $P \in S$, then every morphism $\xi: P \rightarrow M$ factors through $\phi_{0}$, and for every $\psi: P \rightarrow M_{a}$ such that $\phi_{a} \psi=0$ one has $i_{a} \psi=0$. Set $M_{I}:=\operatorname{hocolim}\left(M_{a}, i_{a}\right)$; let $\phi: M_{I} \rightarrow M$ be a morphism such that $\phi \mu_{a}=\phi_{a}$. Then $M_{I^{\perp}}:=\mathcal{C}$ one $(\phi) \in S^{\perp}=I^{\perp}$, so $M_{I} \stackrel{\phi}{\rightarrow} M \rightarrow M_{I^{\perp}}$ is a right $I$-localization triangle.

It remains to construct such a $\left(M_{a}, i_{a}, \phi_{a}\right)$ with $\left(M_{a}, i_{a}\right)$ as in (iv). We do this by induction. Choose $\phi_{0}: M_{0} \rightarrow M$ so that $M_{0}$ is a direct sum of objects in $S$ and every $\xi$ as above factor through $\phi_{0}$. Given $\phi_{a}: M_{a} \rightarrow M$, we define $M_{a+1}$ as $\mathcal{C}$ one $\left(\eta_{a}: C_{a} \rightarrow M_{a}\right)$ where $C_{a}$ is a direct sum of objects in $S, \phi_{a} \eta_{a}=0$, and every $\psi$ as above factors through $\eta_{a}$. Then $i_{a}: M_{a} \rightarrow M_{a+1}$ is the evident morphism, and $\phi_{a+1}$ is any morphism $M_{a+1} \rightarrow M$ such that $\phi_{a+1} i_{a}=\phi_{a}$.

(b) Let us show that for $M \in I$ every morphism $\theta: N \rightarrow M$ with $N \in D^{\text {perf }} \cup I^{\text {perf }}$ factors through an object of $I^{\circ}$.

Let $J \subset I$ be the subcategory of $M$ 's that satisfy this property. It contains $I^{o}$ and is closed under translations and arbitrary direct sums. It remains to check that $J$ is closed under cones. Let $\Delta_{M}=\left(M \rightarrow M^{\prime} \rightarrow M^{\prime \prime}\right)$ be an exact triangle with $M^{\prime}, M^{\prime \prime} \in J$; we want to show that $M \in J$. The composition $N \stackrel{\theta}{\rightarrow} M \rightarrow M^{\prime}$ factors through some $N^{\prime} \in I^{o}$, so there is an exact triangle $\Delta_{N}=\left(N \rightarrow N^{\prime} \rightarrow N^{\prime \prime}\right)$ and a morphism of triangles $(\theta, \alpha, \beta): \Delta_{N} \rightarrow \Delta_{M}$. Since $N^{\prime \prime} \in D^{\text {perf }} \cup I^{\text {perf }}$, one can write $\beta$ as composition $N^{\prime \prime} \stackrel{\gamma}{\rightarrow} L \stackrel{\delta}{\rightarrow} M^{\prime \prime}$ where $L \in I^{o}$. There is an exact triangle $\Delta_{L}=\left(K \rightarrow N^{\prime} \rightarrow L\right)$ and morphisms of triangles $\Delta_{N} \stackrel{\left(\epsilon, \text { id }_{N^{\prime}}, \gamma\right)}{\longrightarrow} \Delta_{L} \stackrel{(\xi, \alpha, \delta)}{\longrightarrow} \Delta_{M}$. The composition $N^{\prime \prime}[-1] \rightarrow N \stackrel{\theta-\xi \epsilon}{\longrightarrow} M$ (the first arrow comes from $\Delta_{N}$ ) vanishes, so $\theta-\xi \epsilon$ factors through $N^{\prime} \in I^{o}$. Thus $\theta$ factors through $N^{\prime} \oplus K \in I^{o}$, q.e.d.

(c) Applying (b) to $\operatorname{id}_{N}, N \in I^{\text {perf }}$, we see that $I^{\text {perf }}=\left(I^{o}\right)^{\kappa}$; we have proved (i). Since the direct sum in $D$ of an arbitrary family of objects of $I^{\perp}$ lies in $S^{\perp}=I^{\perp}$, we get (ii). Let us prove (iii). It follows from (b) that the functor $D^{\text {perf }} / I^{\text {perf }} \rightarrow(D / I)^{\text {perf }} \subset D / I$ is fully faithful. By (i) (with $I$ replaced by $D$ ), $D^{\text {perf }}$ is essentially small. Its image generates $D / I$, so by (i) (with $I$ replaced by $D / I, S$ by $\left.j^{*}\left(D^{\text {perf }}\right)\right),\left(D^{\text {perf }} / I^{\text {perf }}\right)^{\kappa}=\left\langle j^{*}\left(D^{\text {perf }}\right)\right\rangle^{\kappa}=(D / I)^{\text {perf }}$, q.e.d.

1.4.3. Suppose $D$ is a cocomplete triangulated category and $I_{1}^{o}, I_{2}^{o} \subset D^{\text {perf }}$ 
are two small full triangulated subcategories. Let $I_{1}, I_{2} \subset D$ be the triangulated subcategories generated by $I_{1}^{o}, I_{2}^{o}$. By 1.4.2, $I_{1}, I_{2}$ are right admissible.

Proposition. $I_{1}, I_{2}$ are compatible if and only if the following condition holds: Every morphism $\phi: M_{1} \rightarrow M_{2}$, where $M_{i} \in I_{i}^{o}$, can be represented as a composition $M_{1} \rightarrow N_{2} \rightarrow N_{1} \rightarrow M_{2}$ with $N_{i} \in I_{i}^{o}$, and the same is true if we interchange $I_{i}^{o}$.

Proof. (i) Suppose $I_{1}, I_{2}$ are compatible; let us check the condition. Write $\phi$ as the composition $M_{1} \stackrel{\psi}{\rightarrow} P \rightarrow M_{2}$ where $P:=i_{1 *} i_{1}^{!} M_{2}$. Since $P \in I_{2}$ and $M_{1} \in D^{\text {perf }}$, by part (b) of the proof in 1.4 .2 , we can write $\psi$ as a composition $M_{1} \rightarrow N_{2} \stackrel{\chi}{\rightarrow} P$ with $N_{2} \in I_{2}^{o}$. Similarly, since $P \in I_{1}$ and $N_{2} \in D^{\text {perf }}, \chi$ can be written as a composition $N_{2} \rightarrow N_{1} \rightarrow P$ with $N_{1} \in I_{1}^{o}$, and we are done.

(ii) Suppose the condition holds; let us show that $I_{1}, I_{2}$ are compatible. By 1.3(iii) ${ }^{\prime}$, it suffices to check that for any $F \in I_{2}$ the object $P:=i_{1 *} i_{1}^{!} F$ lies in $I_{2}$. We will prove that any morphism $\theta: M \rightarrow P$ with $M \in D^{\text {perf }}$ factors through an object of $I_{2}$. This implies the claim. Indeed, replacing $D$ by the subcategory generated by $I_{1}, I_{2}$, we can assume that $D$ is generated by $D^{\text {perf }}$. By 1.4.2(ii), $P \in I_{2}$ iff for any $M^{\prime} \in D^{\text {perf }}$ one has $\operatorname{Hom}_{D / I_{2}}\left(M^{\prime}, P\right)=0$. Any morphism $M^{\prime} \rightarrow P$ in $D / I_{2}$ can be lifted to a morphism $\theta: M \rightarrow P$ in $D$, where $M:=\mathcal{C}$ one $(\xi)[-1]$ for some $\xi: M^{\prime} \rightarrow N, N \in I_{2}$; by part (b) of the proof in 1.4.2, we can assume that $N \in I_{2}^{o}$, so $M \in D^{\text {perf }}$, and $\theta$ vanishes in $D / I_{2}$ by the assertion, q.e.d.

By part (b) of the proof in 1.4.2, our $\theta: M \rightarrow P$ factors through some $M_{1} \in I_{1}^{o}$, and the composition $M_{1} \stackrel{\theta}{\rightarrow} P \rightarrow F$ factors through some $M_{2} \in I_{2}^{o}$. Let us factorize the map $M_{1} \rightarrow M_{2}$ as in the condition. By the universal property, the composition $N_{1} \rightarrow M_{2} \rightarrow F$ factors through $P$, and $M_{1} \rightarrow P$ equals the composition $M_{1} \rightarrow$ $N_{2} \rightarrow N_{1} \rightarrow P$. We have factorized $\theta$ as $M \rightarrow N_{2} \rightarrow P$, q.e.d.

1.5. DG categories. With respect to triangulated categories, DG categories play the same role as topological spaces for objects of the homotopy category.

1.5.1. Let $\mathcal{A}$ be a DG $(R$-)category. Thus morphisms between objects of $\mathcal{A}$ are complexes of $R$-modules, and the composition maps are morphisms of complexes. The homotopy category Hot $\mathcal{A}$ and the graded homotopy category Hot $\mathcal{A}$ have same objects as $\mathcal{A}$, and morphisms $\operatorname{Hot}\left(M, M^{\prime}\right):=\operatorname{Hom}_{\operatorname{Hot} \mathcal{A}}\left(M, M^{\prime}\right)=H^{0} \operatorname{Hom}\left(M, M^{\prime}\right)$, $\operatorname{Hot}\left(M, M^{\prime}\right):=\operatorname{Hom}_{\text {Hot }} \mathcal{A}\left(M, M^{\prime}\right)=\oplus H^{n} \operatorname{Hom}\left(M, M^{\prime}\right)$. A $D G$ morphism $\phi$ in $\mathcal{A}$ is a closed morphism of degree 0 . Such $\phi$ yields a morphism in Hot $\mathcal{A}$; if the latter is an isomorphism, then $\phi$ is said to be homotopy equivalence.

Our $\mathcal{A}$ is $D G$ Karoubian if it contains the image of any idempotent DG endomorphism. For an arbitrary $\mathcal{A}$ we denote by $\mathcal{A}^{\kappa}$ its DG idempotent completion (defined in the evident way), which is a DG Karoubian DG category.

If $\mathcal{A}, \mathcal{B}$ are $\mathrm{DG}$ categories, then a DG ( $R$-linear) functor $F: \mathcal{A} \rightarrow \mathcal{B}$ yields functors $\operatorname{Hot} F: \operatorname{Hot} \mathcal{A} \rightarrow \operatorname{Hot} \mathcal{B}$, $\operatorname{Hot}^{\prime} F: \operatorname{Hot} \mathcal{A} \rightarrow \operatorname{Hot} \mathcal{B}$. Our $F$ is a quasiequivalence if $\operatorname{Hot} F$ is a fully faithful functor and $\operatorname{Hot} F$ is essentially surjective.

Examples. (i) An associative unital DG ( $R$-)algebra $A$ is the same as a DG category with single object; we denote the latter also by $A$.

(ii) For any $R$-category $\mathcal{A}$ the category $C \mathcal{A}$ of complexes in $\mathcal{A}$ is a DG category. 
1.5.2. A homotopy $D G$ functor $\tilde{F}=(\tilde{F}, \iota): \mathcal{A} \rightarrow \mathcal{B}$ is a diagram $\mathcal{A} \stackrel{\iota}{\leftarrow} \stackrel{\tilde{\mathcal{F}}}{\longrightarrow} \mathcal{B}$ of DG functors such that $\iota$ is a quasi-equivalence. If $\mathcal{A}=R$, then this referred to as homotopy object of $\mathcal{B}$ (ordinary objects are the same as plain DG functors $R \rightarrow \mathcal{B}$ ).

Remark. $\tilde{F}$ yields a well-defined functor $\operatorname{Hot} \tilde{F}: \operatorname{Hot} \mathcal{A} \rightarrow \operatorname{Hot} \mathcal{B}$. E.g. a homotopy object of $\mathcal{B}$ yields a well-defined object of Hot $\mathcal{B}$. The converse is not true.

Homotopy DG functors can be composed: the composition of $\mathcal{A} \stackrel{\iota}{\leftarrow} \stackrel{\tilde{\mathcal{F}}}{\longrightarrow} \mathcal{B}$ and $\mathcal{B} \stackrel{\pi^{\prime}}{\leftarrow} \tilde{\mathcal{B}} \stackrel{\tilde{G}}{\longrightarrow} \mathcal{C}$ is $\mathcal{A} \leftarrow \tilde{\mathcal{A}} \stackrel{\tilde{H}}{\longrightarrow} \mathcal{C}$, where $\tilde{\mathcal{A}}^{\prime}$ is the "homotopy fibered product" of $\tilde{\mathcal{A}}$ and $\tilde{\mathcal{B}}$ over $\mathcal{B}$. Namely, objects of $\tilde{\mathcal{A}}^{\prime}$ are triples $(P, Q, \ell)$ where $P \in \tilde{\mathcal{A}}, Q \in \tilde{\mathcal{B}}$, $\ell: \tilde{F}(P) \rightarrow \pi^{\prime}(Q)$ is a homotopy equivalence; $\operatorname{Hom}^{n}\left((P, Q, \ell),\left(P^{\prime}, Q^{\prime}, \ell^{\prime}\right)\right)$ consists of triples $(\phi, \psi, \tau), \phi \in \operatorname{Hom}^{n}\left(P, P^{\prime}\right), \psi \in \operatorname{Hom}^{n}\left(Q, Q^{\prime}\right), \tau \in \operatorname{Hom}^{n-1}\left(\tilde{F}(P), \pi^{\prime}\left(Q^{\prime}\right)\right)$; the differential on Hom's is determined by the property that natural functors $\tilde{\mathcal{A}}^{\prime} \rightarrow$ $\tilde{\mathcal{A}}, \tilde{\mathcal{B}}, \mathcal{B},(P, Q, \ell) \mapsto P, Q, \mathcal{C}$ one $(\ell)$, are DG functors.

1.5.3. Two DG categories $\mathcal{A}, \mathcal{B}$ yield $\mathrm{DG}$ categories $\mathcal{A} \otimes \mathcal{B}=\mathcal{A} \otimes_{R} \mathcal{B}$ and (if $\mathcal{A}$ is essentially small) $\mathcal{H o m}(\mathcal{A}, \mathcal{B})$. Namely, objects of $\mathcal{A} \otimes \mathcal{B}$ are written as $M \otimes N$ where $M \in \mathcal{A}, N \in \mathcal{B}$, and $\operatorname{Hom}\left(M \otimes N, M^{\prime} \otimes N^{\prime}\right)=\operatorname{Hom}\left(M, M^{\prime}\right) \otimes \operatorname{Hom}\left(N, N^{\prime}\right)$. Objects of $\operatorname{Hom}(\mathcal{A}, \mathcal{B})$ are DG functors $F: \mathcal{A} \rightarrow \mathcal{B}$; the complex $\operatorname{Hom}\left(F, F^{\prime}\right)$ has components $\operatorname{Hom}^{n}\left(F, F^{\prime}\right)$ formed by degree $n$ morphisms of graded functors, the differential is evident. There is an "evaluation" DG functor $\mathcal{H o m}(\mathcal{A}, \mathcal{B}) \otimes \mathcal{A} \rightarrow \mathcal{B}$, $F \otimes M \mapsto F(M)$, which satisfies a universality property: if $\mathcal{C}$ is another essentially small DG category, then $\operatorname{Hom}(\mathcal{C}, \mathcal{H o m}(\mathcal{A}, \mathcal{B}))=\mathcal{H o m}(\mathcal{C} \otimes \mathcal{A}, \mathcal{B})$. We get:

Lemma. Small DG categories form a tensor category $\mathcal{D G}$ with inner Hom's.

Remark. While $\otimes$ preserves quasi-equivalences (when one of the arguments is homotopically $R$-flat), this is not true for $\mathcal{H o m}$, even if $\mathcal{A}$ is semi-free in the sense of $[\mathrm{Dr}] \mathrm{B} 4$, for $\mathcal{H o m}(\mathcal{A}, \mathcal{B})$ has too few morphisms. As shown in $[\mathrm{T}]$, replacing strict morphisms between DG functors by lax ones, one gets a natural 2-(DG)category structure compatible with the homotopy localization.

1.5.4. Let $\mathcal{A}$ be a DG category. For $M \in \mathcal{A}$ its $n$-translation, $n \in \mathbb{Z}$, is an object $M[n]$ of $\mathcal{A}$ representing the DG functor $N \mapsto \operatorname{Hom}(N, M)[n]$. Similarly, for a DG morphism $\phi: M \rightarrow M^{\prime}$ its cone is an object $\operatorname{Cone}(\phi)$ of $\mathcal{A}$ that represents the DG functor $N \mapsto \mathcal{C}$ one $\left(\operatorname{Hom}(N, M) \stackrel{\phi}{\rightarrow} \operatorname{Hom}\left(N, M^{\prime}\right)\right)$. Our $\mathcal{A}$ is strongly pretriangulated if $\mathcal{A}$ contains 0 and $M[n], \mathcal{C}$ one $(\phi)$ exist for every $M, n$, and $\phi$.

For every $\mathcal{A}$ there is a fully faithful DG embedding $\mathcal{A} \hookrightarrow \mathcal{A}^{\text {pretr }}$ such that $\mathcal{A}^{\text {pretr }}$ is strongly pretriangulated and the smallest strongly pretriangulated DG subcategory that contains $\mathcal{A}$ equals $\mathcal{A}^{\text {pretr }}$. Such an embedding is essentially unique: more precisely, if $\mathcal{B}$ is strongly pretriangulated, then every DG functor $F: \mathcal{A} \rightarrow \mathcal{B}$ extends in a unique, up to a unique DG isomorphism, way to a DG functor $F^{\text {pretr }}$ : $\mathcal{A}^{\text {pretr }} \rightarrow \mathcal{B}$. For a concrete construction of $\mathcal{A}^{\text {pretr }}$, see Remark (i) in 1.6.1.

A DG category $\mathcal{A}$ is said to be pretriangulated if $\operatorname{Hot} \mathcal{A} \rightarrow \operatorname{Hot} \mathcal{A}^{\text {pretr }}$ is an equivalence of categories. If this happens, then $\operatorname{Hot} \mathcal{A}$ is naturally a triangulated category $^{2}($ see $[\mathrm{BK}])$. For an arbitrary $\mathcal{A}$, we denote by $\mathcal{A}^{\text {tri }}$ the triangulated

\footnotetext{
${ }^{2}$ To see this, notice that the Yoneda embedding $\mathcal{A} \hookrightarrow \mathcal{A}^{\mathrm{op}}$-dgm (see 1.6.3) identifies Hot $\mathcal{A}$ with
} 
category Hot $\mathcal{A}^{\text {pretr }}$. Any DG functor $F: \mathcal{A} \rightarrow \mathcal{B}$ yields a triangulated functor $F=F^{\text {tri }}: \mathcal{A}^{\text {tri }} \rightarrow \mathcal{B}^{\text {tri }}$.

Lemma. If $F$ is a quasi-equivalence, then such is $F^{\text {pretr }}$.

Example. If $\mathcal{A}$ is an additive category considered as a DG category, then $\mathcal{A}^{\text {pretr }}$ is the DG category $C^{b} \mathcal{A}$ of bounded complexes in $\mathcal{A}$, and $\mathcal{A}^{\text {tri }}$ is the bounded homotopy category $K^{b} \mathcal{A}$.

1.5.5. For a triangulated category $D$, a $D G$ structure on $D$ is a pretriangulated DG category $\mathcal{A}=\mathcal{A}_{D}$ together with an equivalence of triangulated categories $\mathcal{A}^{\text {tri }} \stackrel{\sim}{\rightarrow} D$. It is clear what a $D G$ lifting of a triangulated functor between triangulated categories equipped with DG structures is. A DG structure on $D$ automatically induces a DG structure on any (strictly) full triangulated subcategory $I \subset D$ : take for $\mathcal{A}_{I}$ the preimage of $I$ in $\mathcal{A}$. If $I$ is right admissible, then $\mathcal{A}_{I^{\perp}}$ is a DG structure on $D / I$ called the (right) $D G$ quotient $D G$ structure ${ }^{3}$ and denoted by $\mathcal{A} / \mathcal{A}_{I}$.

Remark. The projection $D \rightarrow D / I$ lifts naturally to a homotopy $D G$ functor $\mathcal{A} \leftarrow \tilde{\mathcal{A}} \rightarrow \mathcal{A} / \mathcal{A}_{I}$. Namely, an object of $\tilde{\mathcal{A}}$ is a DG morphism $\phi: M \rightarrow N$ in $\mathcal{A}$ such that $N \in \mathcal{A}_{I^{\perp}}$, Cone $(\phi) \in \mathcal{A}_{I}$, and the complex $\operatorname{Hom}_{\tilde{\mathcal{A}}}\left(\phi, \phi^{\prime}\right)$ is the subcomplex of $\operatorname{Hom}\left(\operatorname{Cone}(\phi), \mathcal{C}\right.$ one $\left.\left(\phi^{\prime}\right)\right)$ formed by those morphisms that map $N \subset \mathcal{C}$ one $(\phi)$ to $N^{\prime} \subset \mathcal{C}$ one $\left(\phi^{\prime}\right)$; the above DG functors send $\phi$ to $M$ and $N$.

1.5.6. The proposition below is inspired by $\S 6$ of $[\mathrm{Bo} 1] .{ }^{4}$

Let $D$ be a triangulated category and $B \subset D$ be a full subcategory closed under finite direct sums which strongly generates $D$. Suppose that for every $M, N \in B$ and $n>0$ one has $\operatorname{Hom}(M, N[n])=0$. Let $\mathcal{A}^{\text {tri }} \stackrel{\sim}{\rightarrow} D$ be a DG structure on $D$.

Proposition. (i) There is a natural homotopy DG functor $\epsilon: \mathcal{A} \rightarrow C^{b} B$.

(ii) The functor $\epsilon^{\text {tri }}: D \rightarrow K^{b} B$ is conservative. For $D$ essentially small, it yields an isomorphism of the Grothendieck groups $K_{0}(D) \stackrel{\sim}{\rightarrow} K_{0}\left(K^{b} B\right)=K_{0}(B)$.

(iii) If $B$ is Karoubian, then $D$ is Karoubian.

Proof. (i) Let $\mathcal{B}$ be a DG subcategory of $\mathcal{A}$ formed by those $M \in \mathcal{A}$ that are isomorphic to objects of $B$ in $D$, and $\operatorname{Hom}_{\mathcal{B}}\left(M, M^{\prime}\right):=\tau_{\leq 0} \operatorname{Hom}_{\mathcal{A}}\left(M, M^{\prime}\right)$. Then $\mathcal{B}^{\text {tri }} \stackrel{\sim}{\rightarrow} \mathcal{A}^{\text {tri }} \stackrel{\sim}{\rightarrow} D$. So we can assume that $\mathcal{A}=\mathcal{B}^{\text {pretr }}$.

One has a DG functor $\epsilon_{0}: \mathcal{B} \rightarrow B$ which is the identity on objects and is the projection $\operatorname{Hom}_{\mathcal{B}}\left(M, M^{\prime}\right)=\tau_{\leq 0} \operatorname{Hom}_{\mathcal{B}}\left(M, M^{\prime}\right) \rightarrow H^{0} \operatorname{Hom}_{\mathcal{B}}\left(M, M^{\prime}\right)$ on morphisms. Set $\epsilon:=\epsilon_{0}^{\text {pretr }}: \mathcal{B}^{\text {pretr }} \rightarrow B^{\text {pretr }}=C^{b} B$.

(ii) By Remark (i) in 1.6.1, every $M \in \mathcal{A}=\mathcal{B}^{\text {pretr }}$ can be written as $\left(\oplus M^{i}[-i], \phi\right)$ where $M^{i} \in \mathcal{B}$. The components $\phi_{i}^{j} \in \operatorname{Hom}^{i-j+1}\left(M^{i}, M^{j}\right)$ vanish for $i \geq j$, so

the full subcategory of the homotopy category of right DG $\mathcal{A}$-modules, which is triangulated by the standard argument. If $\mathcal{A}$ is pretriangulated, then $\operatorname{Hot} \mathcal{A}$ is closed under cones and translations.

${ }^{3}$ The construction is not self-dual: it is adapted for handling the right derived functors.

${ }^{4}$ For more general results in this vein see [Bo2]. We are grateful to M. Bondarko for sending us a draft of the article and relevant correspondence. 
one has a finite decreasing filtration $F^{a} M:=\oplus_{i \geq a} M^{i}[-i]$ on $M$. Every morphism $\theta: M \rightarrow M^{\prime}$ of degree 0 is compatible with $F$; we write $\theta^{i}:=\operatorname{gr}_{F}^{i} \theta \in \operatorname{Hom}\left(M^{i}, M^{i}\right)$. The complex $\epsilon(M)$ has components $M^{i} \in B$, its differential $d \in \operatorname{Hom}_{B}\left(M^{i}, M^{i+1}\right)$ is the class of $\phi_{i}^{i+1}$ in $H^{0} \operatorname{Hom}\left(M^{i}, M^{i+1}\right)$.

$\epsilon^{\text {tri }}$ is conservative: Suppose $\epsilon(M)$ is contractible. This means that there are decompositions $M^{i}=L^{i} \oplus L^{i+1}$ in $B$ such that $d_{i}$ equals the composition of the projection and the embedding $M^{i} \rightarrow L^{i+1} \hookrightarrow M^{i+1}$. A downward induction by $a$ shows that the composition $F^{a} M \rightarrow M^{a}[-a] \rightarrow L^{a}[-a]$ is a homotopy equivalence. For $a \ll 0$ we get $0=F^{a} M=M$, q.e.d.

$K_{0}\left(\epsilon^{\mathrm{tri}}\right)$ is an isomorphism: Since $B$ strongly generates $D, K_{0}(D)$ is generated by classes $[M], M \in B$. The relation $\left[M \oplus M^{\prime}\right]=[M]+\left[M^{\prime}\right]$ evidently holds in $K_{0}(D)$. Since $K_{0}(B)$ is universal with respect to these generators and relations, we get a map $K_{0}\left(K^{b} B\right)=K_{0}(B) \rightarrow K_{0}(D)$ which is evidently inverse to $K_{0}\left(\epsilon^{\text {tri }}\right)$.

(iii) Suppose $B$ is Karoubian; let us show that every $P \in D^{\kappa}$ lies in $D$.

Choose $M \in \mathcal{A}$ and an idempotent $p \in H^{0} \operatorname{End} M$ whose image equals $P$. Write $M=\left(\oplus M^{i}[-i], \phi\right)$ as above. Shifting $M$, we can assume that $F^{n} M=0, F^{1} M=M$ for some odd $n \geq 1$. By [Th] (see Remark (i) in 1.1.1), $P[n] \oplus P$ lies in $D$ for every odd $n .^{5}$ Choose $N=\left(\oplus N^{i}[-i], \psi\right) \in \mathcal{A}$ that represents $P[n] \oplus P$. The projectors $\bar{p}, \bar{p}^{\prime} \in H^{0}$ End $N$ onto $P, P[n]$ factor through, respectively, $M$ and $M[n]$, so one can represent them by DG endomorphisms $p, p^{\prime}$ of $N$ such that $p$ factors through $M$ and $p^{\prime}$ factors through $M[n]$.

Consider the complex $\epsilon(N) \in C^{b} B$. Its identity map is homotopic to $\epsilon(p)+$ $\epsilon\left(p^{\prime}\right)$; let $h \in \operatorname{Hom}^{0}(\epsilon(N), \epsilon(N)[-1])$, be the homotopy. Set $q:=h^{1} d, q^{\prime}:=d h^{0} \in$ $H^{0} \operatorname{End}\left(N^{0}\right)$. Since $M^{0}=0=(M[n])^{0}$, one has $p^{0}=0=p^{0}=0$. Therefore $\operatorname{id}_{N^{0}}=q+q^{\prime}$ and $q^{2}=h^{1} d\left(h^{1} d+d h^{0}\right)=q, q^{\prime 2}=\left(h^{1} d+d h^{0}\right) d h^{0}=q^{\prime}, q q^{\prime}=0$. The images of the idempotents $q, q^{\prime}$ lie in $B$ since $B$ is Karoubian; let us represent them by $Q, Q^{\prime} \in \mathcal{B}$, so we have an homotopy equivalence $Q \oplus Q^{\prime} \stackrel{\sim}{\rightarrow} N^{0}$.

Let $T$ be the cone of the composition $Q[-1] \rightarrow N^{0}[-1] \stackrel{\psi_{0}}{\longrightarrow} F^{1} N$, so $F^{0} T=$ $T, F^{1} T=F^{1} N$. There is an evident DG morphism $e: T \rightarrow N$ identical on $F^{1}$. We will show that $e$ identifies $T \in D$ with $P$. Since $F^{1} M=M$, there is a DG morphism $t: N \rightarrow T$ such that $e t=p$. It remains to check that te $\in \operatorname{End} T$ is a homotopically invertible. Now $\epsilon(e)$ identifies $\epsilon(T)$ with a subcomplex of $\epsilon(N)$, and $h^{>0}$ is a homotopy between $\epsilon(t e)$ and $\operatorname{id}_{\epsilon(T)}$. Thus $\epsilon(t e)=\mathrm{id}_{\epsilon(T)}$, and we are done by the conservativity of $\epsilon$.

Remark. Probably, statement (iii) of the proposition is valid without the assumption of existence of DG structure on $D$.

1.6. Semi-free modules. 1.6.1. Let $\mathcal{A}$ be a DG category. To perform homotopy constructions on the DG level, one often needs to enlarge $\mathcal{A}$ to a DG category $\underline{\mathcal{A}}$ of "DG ind-objects" defined as follows.

Let $\mathcal{A}^{\natural}$ be a DG category whose objects are formal direct sums $M=\oplus M_{i}\left[n_{i}\right]$, where $i$ runs over some set $I, M_{i} \in \mathcal{A}, n_{i} \in \mathbb{Z}$; one has $\operatorname{Hom}\left(\oplus M_{i}\left[n_{i}\right], \oplus M_{j}^{\prime}\left[n_{j}\right]\right):=$

${ }^{5}$ A direct proof: If $Q \oplus P \in D$, then $P[1] \oplus P=\mathcal{C}$ one $(Q \oplus P \rightarrow Q \oplus P) \in D$, then $Q[2] \oplus P=$ Cone $((Q \oplus P)[1] \rightarrow(P[1] \oplus P)) \in D$, then $P[3] \oplus P=\mathcal{C}$ one $((Q \oplus P)[2] \rightarrow(Q[2] \oplus P)) \in D$, etc. 
$\prod_{i \in I} \underset{j \in J}{\oplus} \operatorname{Hom}\left(M_{i}, M_{j}^{\prime}\right)\left[n_{j}-n_{i}\right]$, the composition law is the product of matrices (with finitely many non-zero entries in each column). An endomorphism $\phi$ of $M$ is said to be $f$-nilpotent if $I$ admits a filtration $I_{0} \subset I_{1} \subset \ldots$ such that $\cup I_{a}=I$ and the corresponding filtration $M_{0} \subset M_{1} \subset \ldots, M_{a}:=\underset{i \in I_{a}}{\oplus} M_{i}\left[n_{i}\right]$, of $M$ has property $\phi\left(M_{a}\right) \subset M_{a-1}, \phi\left(M_{0}\right)=0$. Now $\underline{\mathcal{A}}$ is formed by pairs $(M, \phi)$ where $M \in \mathcal{A}^{\natural}$ and $\phi$ is its f-nilpotent endomorphism of degree 1 which satisfies the Maurer-Cartan equation $d(\phi)+\phi \cdot \phi=0$; the complex $\operatorname{Hom}\left(\left(M, \phi_{M}\right),\left(M^{\prime}, \phi_{M^{\prime}}\right)\right)$ equals $\operatorname{Hom}\left(M, M^{\prime}\right)$ as a $\mathbb{Z}$-graded module, its differential $d_{\phi}$ is $d_{\phi}(f):=d(f)+[\phi, f]$. We usually denote $(M, \phi)$ simply by $M$, and write $\phi_{M}$ for $\phi$.

The DG category $\underline{\mathcal{A}}$ is cocomplete and strongly pretriangulated. One has a fully faithful embedding $\mathcal{A} \hookrightarrow \underline{\mathcal{A}}$ which identifies $M \in \mathcal{A}$ with $(M[0], 0) \in \underline{\mathcal{A}}$.

Remarks. (i) The essential image of the corresponding fully faithful embedding $\mathcal{A}^{\text {pretr }} \hookrightarrow \underline{\mathcal{A}}$ is formed by those $(M, \phi)$ that the sum $M=\oplus M_{i}\left[n_{i}\right]$ is finite. Replacing $\mathcal{A}$ by $\mathcal{A}^{\text {pretr }}$ does not change $\underline{\mathcal{A}}$.

(ii) The embedding $\mathcal{A} \hookrightarrow \underline{\mathcal{A}}$ does not commute with infinite direct sums. Namely, let $\left\{M_{\alpha}\right\}$ be an infinite family of non-zero objects of $\mathcal{A}$ such that the direct sum of $M_{\alpha}$ 's in $\mathcal{A}$ exists; denote it by $\oplus_{\mathcal{A}} M_{\alpha} \in \mathcal{A}$, and let $\oplus M_{\alpha}$ be the direct sum in $\underline{\mathcal{A}}$. Then the canonical morphism $\oplus M_{\alpha} \rightarrow \oplus_{\mathcal{A}} M_{\alpha}$ is not an isomorphism. Same is true if we replace $\mathcal{A}$ and $\underline{\mathcal{A}}$ by their homotopy categories.

Exercise. Suppose $\mathcal{A}^{\text {tri }}$ has arbitrary direct sums and is generated by a set of compact objects. Then the essential image of $\mathcal{A}^{\text {tri }} \rightarrow \underline{\mathcal{A}}^{\text {tri }}$ consists of those $M \in \underline{\mathcal{A}}$ that for every set of objects $N_{i} \in \mathcal{A}$, the canonical morphism $\operatorname{Hom}\left(\bigoplus_{\mathcal{A}^{\text {tri }}} N_{i}, M\right) \rightarrow$ $\prod \operatorname{Hom}\left(N_{i}, M\right)$ is an isomorphism. ${ }^{6}$

Lemma. If a $D G$ category $\mathcal{B}$ is cocomplete, strongly pretriangulated, and $D G$ Karoubian, then every $D G$ functor $F: \mathcal{A} \rightarrow \mathcal{B}$ extends uniquely (up to a unique $D G$ isomorphism) to a $D G$ functor $\underline{F}: \underline{\mathcal{A}} \subset(\underline{\mathcal{A}})^{\kappa} \rightarrow \mathcal{B}$ that commutes with arbitrary direct sums.

Proof. The above $\mathcal{A}^{\natural}$ is a full DG subcategory of $\underline{\mathcal{A}}$ formed by objects $(M, 0)$. Our $F$ extends uniquely to a DG functor $A^{\natural} \rightarrow \mathcal{B}$ which commutes with arbitrary direct sums, and then to $\mathcal{A}^{\natural \operatorname{pretr}} \subset \underline{\mathcal{A}}$. Let $A^{\sim} \subset \underline{\mathcal{A}}$ be the full DG subcategory formed by arbitrary direct sums of objects of $\mathcal{A}^{\natural \text { pretr }}$. Then $F$ extends uniquely to a DG functor $A^{\sim} \rightarrow \mathcal{B}$ which commutes with arbitrary direct sums, hence to $A^{\sim \text { pretr }}$. To finish the proof, it remains to show that $\left(\mathcal{A}^{\sim \operatorname{pretr}}\right)^{\kappa} \stackrel{\sim}{\rightarrow}(\underline{\mathcal{A}})^{\kappa}$. Notice that the construction of hocolim from 1.4.1 lifts in the evident manner to DG level (and it becomes canonical, as cones are). Now for any $M \in \underline{\mathcal{A}}$ the terms of the filtration $M_{0} \subset M_{1} \subset \ldots$ on $M$ (which comes since $\phi_{M}$ is f-nilpotent) lie in $\mathcal{A}^{\natural \text { pretr }}$, hence hocolim $M_{a} \in \mathcal{A}^{\sim \operatorname{pretr}}$. Since the evident projection hocolim $M_{a} \rightarrow M$ admits a DG splitting, one has $M \in\left(\mathcal{A}^{\sim \operatorname{pretr}}\right)^{\kappa}$, q.e.d.

1.6.2. A DG category $\mathcal{A}$ is said to be homotopically Karoubian if Hot $\mathcal{A}$ is Karoubian. For any $\mathcal{A}$ its homotopy idempotent completion is a fully faithful DG embedding $\mathcal{A} \hookrightarrow \mathcal{B}$ such that $\operatorname{Hot} \mathcal{B}$ is the idempotent completion of Hot $\mathcal{A}$. Here

${ }^{6}$ Hint: use (i) of Proposition in 1.4 .2 for $I=\mathcal{A}^{\text {tri }}$ and $D$ formed by $M \in \underline{\mathcal{A}}$ as above. 
is a concrete construction: for $\mathcal{B}$, take a full DG subcategory of $\underline{\mathcal{A}}$ formed by those objects that represent in Hot $\underline{\mathcal{A}}$ direct summands of objects in Hot $\mathcal{A}$; since $\underline{\mathcal{A}}^{\text {tri }}$ is Karoubian by 1.4.1, our $\mathcal{B}$ is a homotopy idempotent completion of $\mathcal{A}$. This construction also shows that all homotopy idempotent completions of $\mathcal{A}$ are naturally quasi-equivalent.

1.6.3. Suppose a DG category $\mathcal{A}$ is essentially small. Denote by $R$-dgm the DG category of complexes of $R$-modules. The objects of DG category $\mathcal{A}$-dgm $:=$ $\mathcal{H o m}(\mathcal{A}, R$-dgm $)$ are called left $(D G) \mathcal{A}$-modules. A right $\mathcal{A}$-module is the same as an $\mathcal{A}^{\mathrm{op}}$-module. $\mathcal{A}^{\mathrm{op}}$-dgm is strongly pretriangulated, cocomplete, and DG Karoubian. One has the Yoneda fully faithful DG embeddings $\mathcal{A} \hookrightarrow \mathcal{A}^{\mathrm{op}}$-dgm, $\mathcal{A}^{\mathrm{op}} \hookrightarrow \mathcal{A}$-mod.

Remarks. (i) If $\mathcal{A}$ has single object, $\mathcal{A}=A$, then $\mathcal{A}$-module $=\mathrm{DG} A$-module.

(ii) There are natural DG functors $\mathcal{A}^{\mathrm{op}}$-dgm $\otimes \mathcal{A}$-dgm $\rightarrow\left(\mathcal{A}^{\mathrm{op}} \otimes \mathcal{A}\right)$-dgm $\rightarrow$ $R$-dgm, the first one assigns to $F \otimes G$ the $\mathcal{A}^{\mathrm{op}} \otimes \mathcal{A}$-module $M \otimes N \mapsto F(M) \otimes_{R} G(N)$, the second one is the coend functor. We denote the composition as $F \otimes G \mapsto F \otimes_{\mathcal{A}} G$. In the situation of (i) this is the usual tensor product of left and right $A$-modules.

The DG functor $\underline{\mathcal{A}} \rightarrow \mathcal{A}^{\mathrm{op}}$-dgm defined by the embedding $\mathcal{A} \hookrightarrow \mathcal{A}^{\mathrm{op}}$-dgm (see the lemma in 1.6.1) is itself a fully faithful embedding whose essential image consists of semi-free $\mathcal{A}^{\mathrm{op}}$-modules. Here an $\mathcal{A}^{\mathrm{op}}$-module $M$ is said to be semi-free if it admits an increasing exhaustive filtration $M_{0} \subset M_{1} \subset \ldots$ such that each $\operatorname{gr}_{a} M$ is free, i.e., isomorphic to a direct sum of translations of objects in $\mathcal{A}$. Thus $M_{a+1}=$ Cone $\left(C_{a} \rightarrow M_{a}\right)$ where $C_{a}$ is a free $\mathcal{A}^{\text {op }}$-module. Replacing $\mathcal{A}$ by $\mathcal{A}^{\text {pretr }}$ does not change these categories.

Lemma. $\underline{\mathcal{A}}^{\text {tri }}=\underline{\mathcal{A}}^{\kappa \text { tri }}$ is a right-admissible subcategory of $\mathcal{A}^{\mathrm{op}}-d g m^{\mathrm{tri}}$.

Proof. See 1.4.1 and (i) of the proposition in 1.4.2.

1.6.4. An $\mathcal{A}^{\mathrm{op}}$-module is said to be acyclic if the functor $\mathcal{A}^{\mathrm{op}} \rightarrow R$-dgm takes values in acyclic complexes. Those objects form a full pretriangulated DG subcategory $\mathcal{A}^{\mathrm{op}}-\mathrm{dgm}_{a c} \subset \mathcal{A}^{\mathrm{op}}-\mathrm{dgm}$, and $\mathcal{A}^{\mathrm{op}}-\mathrm{dgm}_{a c}^{\text {tri }}$ is the right orthogonal complement to $\underline{\mathcal{A}}^{\text {tri }}$. By 1.2 and the above lemma, $\mathcal{A}^{\text {op }}$-dgmac ${ }_{a c}^{\text {tri }}$ is left admissible, its left orthogonal complement equals $\underline{\mathcal{A}}^{\text {tri }}$, and the restriction of the projection $\mathcal{A}^{\mathrm{op}}$-dgm ${ }^{\text {tri }} \rightarrow D\left(\mathcal{A}^{\mathrm{op}}\right):=\mathcal{A}^{\mathrm{op}}$-dgm ${ }^{\text {tri }} / \mathcal{A}^{\mathrm{op}}$-dgm ${ }_{a c}^{\text {tri }}$ (the derived category of right $\mathcal{A}$-modules) to $\underline{\mathcal{A}}^{\text {tri }}$ is an equivalence of categories:

$$
\underline{\mathcal{A}}^{\text {tri }} \stackrel{\sim}{\rightarrow} D\left(\mathcal{A}^{\mathrm{op}}\right) \text {. }
$$

Thus $\underline{\mathcal{A}}$ is a DG structure on $D\left(\mathcal{A}^{\mathrm{op}}\right)$; we refer to it as the canonical $D G$ structure.

Remark. The procedure from (a) of the proof of the proposition in 1.4 .2 (performed on DG level) provides for any $M \in \mathcal{A}$-dgm its semi-free left resolution $\operatorname{hocolim}\left(M_{a}, i_{a}\right) \rightarrow M$. In fact, one can replace hocolim by the plain direct limit $\varliminf M_{a}$ (which is semi-free): the morphism hocolim $\left(M_{a}, i_{a}\right) \rightarrow M$ factors through $\varliminf M_{a}$, and $\operatorname{hocolim}\left(M_{a}, i_{a}\right) \rightarrow \varliminf_{a} M_{a}$ is a homotopy equivalence.

A DG functor $F: \mathcal{A}_{1} \rightarrow \mathcal{A}_{2}$ yields an evident DG functor $F_{*}: \mathcal{A}_{2}^{\mathrm{op}}$-dgm $\rightarrow$ $\mathcal{A}_{1}^{\mathrm{op}}$-dgm and its left adjoint $F^{*}: \mathcal{A}_{1}^{\mathrm{op}}$-dgm $\rightarrow \mathcal{A}_{2}^{\mathrm{op}}$-dgm that extends $F$ and sends $\underline{\mathcal{A}_{1}}$ to $\underset{\mathcal{A}_{2}}{\longrightarrow}$; they commute with arbitrary direct sums. Since $F_{*}$ preserves acyclic 
modules (or quasi-isomorphisms), it yields a triangulated functor $F_{*}: D\left(\mathcal{A}_{2}^{\mathrm{op}}\right) \rightarrow$ $D\left(\mathcal{A}_{1}^{\text {op }}\right)$ whose left adjoint comes via (1.6.1) from $F^{*}:{\underline{\mathcal{A}_{1}}}^{\text {tri }} \rightarrow{\underline{\mathcal{A}_{2}}}^{\text {tri }}$.

Lemma. If $F$ yields an equivalence $\mathcal{A}_{1}^{\text {tri } \kappa} \stackrel{\sim}{\rightarrow} \mathcal{A}_{2}^{\text {tri } \kappa}$, then $F^{*}, F_{*}: D\left(\mathcal{A}_{1}^{\mathrm{op}}\right) \leftrightarrows$ $D\left(\mathcal{A}_{2}^{\mathrm{op}}\right)$ are mutually inverse equivalences of triangulated categories.

1.7. Cocomplete vs. small \& Karoubian: DG setting. The next result is due to V. Drinfeld.

Let $\mathcal{B}$ be a cocomplete pretriangulated DG category. An object of $\mathcal{B}$ is said to be perfect if it is perfect (i.e., compact) as an object of $\mathcal{B}^{\text {tri }}$. All perfect objects form a full DG subcategory $\mathcal{B}^{\text {perf }} \subset \mathcal{B}$. E.g., $\mathcal{A} \hookrightarrow \underline{\mathcal{A}}$ takes values in $(\underline{\mathcal{A}})^{\text {perf }}$.

Consider the following two classes of DG categories:

(a) Pretriangulated DG categories $\mathcal{A}$ with $\mathcal{A}^{\text {tri }}$ essentially small and Karoubian.

(b) Cocomplete DG Karoubian strongly pretriangulated DG categories $\mathcal{B}$ such that $\mathcal{B}^{\text {tri }}$ is generated by a set of perfect objects.

They form naturally "DG 2-categories" $\mathcal{D} \mathcal{G}^{(a)}$ and $\mathcal{D} \mathcal{G}^{(b)}$. Namely, 1-morphisms in $\mathcal{D} \mathcal{G}^{(a)}$ are DG functors, 1-morphisms in $\mathcal{D} \mathcal{G}^{(b)}$ are DG functors that commute with arbitrary direct sums and send perfect objects to perfect ones, and the "complex" of 2-morphisms between DG functors is defined as in 1.5.3 (the quotation marks mean that morphisms need not form sets).

For $\mathcal{A}$ of type (a) the category $(\underline{\mathcal{A}})^{\kappa}$ is of type (b); for $\mathcal{B}$ of type (b) $\mathcal{B}^{\text {perf }}$ is of type (a) (see (i) of the proposition in 1.4.2). By the lemma in 1.6.1, we have defined "adjoint DG functors"

$$
\mathcal{D} \mathcal{G}^{(a)} \leftrightarrows \mathcal{D} \mathcal{G}^{(b)}
$$

Proposition. The adjunction 1-morphisms $\mathcal{A} \rightarrow(\underline{\mathcal{A}})^{\kappa \text { perf }},\left(\stackrel{\mathcal{B}^{\text {perf }}}{\longrightarrow}\right)^{\kappa} \rightarrow \mathcal{B}$ are quasi-equivalences.

Proof. The assertion is implied by the next lemma, which follows from 1.4.2:

Lemma. If $\mathcal{A} \subset \mathcal{B}^{\text {perf }}$ is any small full $D G$ subcategory that generates $\mathcal{B}^{\text {tri }}$, then $\mathcal{A} \rightarrow \mathcal{B}$ is a quasi-equivalence, and $\left(\mathcal{B}^{\text {perf }}\right)^{\text {tri }}=\left(\mathcal{A}^{\text {tri }}\right)^{\kappa}$.

Remarks. (i) The proposition asserts that $\mathcal{A} \mapsto \underline{\mathcal{A}}$ and $\mathcal{B} \mapsto \mathcal{B}^{\text {perf }}$ are "homotopically mutually inverse" correspondences between the categories of type (a) and of type (b). Variant: Let us enlarge (b) to the class (b)' of cocomplete pretriangulated DG categories $\mathcal{B}$ such that $\mathcal{B}^{\text {tri }}$ is generated by a set of perfect objects. For any such $\mathcal{B}$ the embedding $\mathcal{B} \hookrightarrow \mathcal{B}^{\text {pretr } \kappa}$ is a quasi-equivalence (see 1.4.1), and $\mathcal{B}^{\text {pretr } \kappa}$ is of type (b). Thus $\mathcal{A} \mapsto \underline{\mathcal{A}}$ and $\mathcal{B} \mapsto \mathcal{B}^{\text {perf }}$ are "homotopically mutually inverse" correspondences between the categories of type (a) and of type (b)'.

(ii) For any essentially small DG category $\mathcal{A}$ the category $\underline{\mathcal{A}}^{\text {perf }}$ is a homotopy idempotent completion of $\mathcal{A}^{\text {pretr }}$, see 1.6.2.

1.8. Homotopy DG quotients. Let $\mathcal{A}$ be a DG category such that $\mathcal{A}^{\text {tri }}$ is essentially small, $\mathcal{I} \subset \mathcal{A}$ be a full DG subcategory. By $1.4 .2, \underline{\mathcal{I}}^{\text {tri }} \subset \underline{\mathcal{A}}^{\text {tri }}$ is a 
right-admissible subcategory. As in 1.5.5, let $\mathcal{B}:=\underline{\mathcal{A}} / \underline{\mathcal{I}} \subset \underline{\mathcal{A}}$ be the preimage of $\left(\underline{\mathcal{I}}^{\text {tri }}\right)^{\perp} \subset \underline{\mathcal{A}}^{\text {tri }}$, so one has $\mathcal{B}^{\text {tri }} \stackrel{\sim}{\rightarrow} \underline{\mathcal{A}}^{\text {tri }} / \underline{\mathcal{I}}^{\text {tri }}$. Let $\mathcal{A} / \mathcal{I} \subset \mathcal{B}$ be the preimage of the essential image of $\mathcal{A}^{\text {tri }}$ in $\underline{\mathcal{A}}^{\text {tri }} / \underline{\mathcal{I}}^{\text {tri }}$; this is the (right) Keller DG quotient. ${ }^{7}$ As in Remark in 1.5.5, one has a natural homotopy DG functor $\mathcal{A} \leftarrow \tilde{\mathcal{A}} \rightarrow \mathcal{A} / \mathcal{I}$, where $(\mathcal{A} / \mathcal{I})^{\text {tri }}=\mathcal{A}^{\text {tri }} / \mathcal{I}^{\text {tri }}{ }^{8}$ We also set $\mathcal{A} /{ }^{\kappa} \mathcal{I}:=\mathcal{B}^{\text {perf }}$, so $\left(\mathcal{A} /{ }^{\kappa} \mathcal{I}\right)^{\text {tri }}=\mathcal{A}^{\text {tri }} /{ }^{\kappa} \mathcal{I}^{\text {tri }}$ (see 1.4.2). By the lemma in 1.7, one has natural quasi-equivalences

$$
\stackrel{\mathcal{A} / \mathcal{I}}{\longrightarrow} \stackrel{\sim}{\rightarrow} \underline{\mathcal{A} /{ }^{\kappa} \mathcal{I}} \stackrel{\sim}{\longrightarrow} \underline{\mathcal{A}} / \stackrel{\mathcal{I}}{\longrightarrow}
$$

Remark. Small DG categories equipped with a marked zero object form naturally a pointed model category (with quasi-equivalences as weak equivalences and surjective functors as fibrations), ${ }^{9}$ see $[\mathrm{Dr}] \mathrm{B} 4-6$. This plain model structure is not useful, for it does not consider morphisms between DG functors (cf. Remark in 1.5.3). For $\mathcal{A}, \mathcal{I}$ as above, Drinfeld [Dr] defines a (homotopy) $D G$ quotient as a homotopy DG functor $\mathcal{A} \leftarrow \tilde{\mathcal{A}} \rightarrow \mathcal{A} / \mathcal{I}$ where the functor $\operatorname{Hot} \mathcal{A} \rightarrow \operatorname{Hot}(\mathcal{A} / \mathcal{I})$ is essentially surjective and yields an equivalence $\mathcal{A}^{\text {tri }} / \mathcal{I}^{\text {tri }} \rightarrow(\mathcal{A} / \mathcal{I})^{\text {tri }}$. Thus $\mathcal{A} / \mathcal{I}$ is a homotopy cone for the morphism $\mathcal{I} \rightarrow \mathcal{A}$. The Keller construction is a concrete way to construct a DG quotient; ${ }^{10}$ for another one see $\S 3$ of $[\mathrm{Dr}]$. For the uniqueness property of homotopy DG quotients see $[\mathrm{T}]$; a weaker fact is in [Dr]1.6.2.

1.9. Homotopy tensor DG categories. For us, a tensor triangulated category is a triangulated category equipped with a tensor structure such that $\otimes$ is an exact bifunctor. Below we discuss DG liftings of this notion.

1.9.1. Let $\mathcal{A}$ be a DG category. It is clear what a $D G$ tensor structure on $\mathcal{A}$ is. ${ }^{11}$ Unfortunately, DG tensor structures are too rigid to pass readily to homotopy DG quotients. The next definition, due to V. Drinfeld, is adapted for the right Keller localization:

A (right) homotopy tensor structure is a pseudo-tensor structure on $\mathcal{A}$ (see [BD] 1.1.3) such that the corresponding pseudo-tensor structure on $\operatorname{Hot} \mathcal{A}$ is actually a tensor structure. Therefore for every finite non-empty set $I$ we have a DG functor $P_{I}: \mathcal{A}^{\mathrm{op} \otimes I} \otimes \mathcal{A} \rightarrow R$-dgm, $\left(\otimes M_{i}\right) \otimes N \mapsto P_{I}\left(\left\{M_{i}\right\}, N\right)$ of $I$-polylinear operations, the operations compose according to a usual format (and $P_{I}=$ Hom if $|I|=1$ ), and $H^{0} P_{I}\left(\left\{M_{i}\right\}, N\right)=\operatorname{Hom}_{\operatorname{Hot} \mathcal{A}}\left(\otimes M_{i}, N\right)$ for a tensor structure on Hot $\mathcal{A}$ uniquely defined by these operations.

Notice that any DG tensor or homotopy tensor structure extends in a unique way to $\mathcal{A}^{\kappa}$ and $\mathcal{A}^{\text {pretr }}$. It yields tensor structures on Hot $\mathcal{A}$ and $\mathcal{A}^{\text {tri }}$; the latter becomes a tensor triangulated category.

Exercise. The tensor product $\operatorname{Hot} \mathcal{A}^{\otimes I} \rightarrow \operatorname{Hot} \mathcal{A}$ lifts naturally to a homotopy functor (see 1.5.2). So for $M_{i} \in \mathcal{A}$ the tensor product $\otimes M_{i}$ is well defined as a homotopy object of $\mathcal{A}$.

\footnotetext{
${ }^{7}$ See $[\mathrm{K}]$ and $[\mathrm{Dr}] \S 4$. Notice that the construction is not self-dual.

${ }^{8}$ So, by 1.5 .2 , one can assign to each object of $\mathcal{A}$ a homotopy object of $\mathcal{A} / \mathcal{I}$.

${ }^{9}$ It has a peculiar property: suspensions of its objects are contractible.

${ }^{10}$ To retain smallness, one should replace $\mathcal{A} / \mathcal{I}$ (which is not small) by a small DG subcategory having the same homotopy category.

${ }^{11}$ This is a tensor structure such that $\otimes: \mathcal{A} \otimes \mathcal{A} \rightarrow \mathcal{A}$ is a $\mathrm{DG}$ functor and the associativity and commutativity constraints are DG morphisms.
} 
Remark. The above definition of homotopy tensor structure is not self-dual: algebras are pleasant to consider, while coalgebras are cumbersome objects. It would be nice to find a definition of homotopy tensor structure free of this nuisance.

1.9.2. Any DG tensor structure on $\mathcal{A}$ extends naturally to $\underline{\mathcal{A}}$ so that $\otimes$ commutes with arbitrary direct sums. Similarly, any homotopy tensor structure on $\mathcal{A}$ extends naturally to $\underline{\mathcal{A}}$, so that $P_{I}\left(\left\{M_{i}\right\}, N\right)$ transforms arbitrary direct sums of $M_{i}$ 's to direct products, and if $M_{i} \in \mathcal{A}$, then it transforms arbitrary direct sums of $N$ 's to direct sums. The lemma in 1.7 implies that $\left(\mathcal{A}^{\text {tri }}\right)^{\kappa}=\left(\mathcal{A}^{\text {perf }}\right)^{\text {tri }}$ is a tensor subcategory of $\underline{\mathcal{A}}^{\text {tri }}$, so $\underline{\mathcal{A}}^{\text {perf }}$ is a homotopy tensor category.

Suppose $\mathcal{A}$ is essentially small. Then any homotopy tensor structure on $\mathcal{A}$ yields, by means of (1.6.1), a tensor structure on $D\left(\mathcal{A}^{\text {op }}\right)$ such that $\mathcal{A}^{\text {tri }} \subset\left(\mathcal{A}^{\text {tri }}\right)^{\kappa}$ are its tensor subcategories.

If $\mathcal{I} \subset \mathcal{A}$ is a full DG subcategory such that $\mathcal{I}^{\text {tri }}$ is an $\otimes$-ideal in $\mathcal{A}^{\text {tri }}$, then $\mathcal{A} / \mathcal{I} \subset \mathcal{A} /{ }^{\kappa} \mathcal{I} \subset \underline{\mathcal{A}} / \underline{\mathcal{I}}$ are homotopy tensor DG subcategories of $\underline{\mathcal{A}}$. Thus the (right) Keller DG quotient inherits naturally a homotopy tensor structure. It lifts the evident tensor structure on $\mathcal{A}^{\text {tri }} / \mathcal{I}^{\text {tri }}$.

1.9.3. For $M_{i} \in \mathcal{A}$ with $i \in I$ as above, we have a left $\mathcal{A}$-module $P_{I}^{\prime}\left(\left\{M_{i}\right\}\right)$, $N \mapsto P_{I}\left(\left\{M_{i}\right\}, N\right)$ (here $\left.N \in \mathcal{A}\right)$. For any right $\mathcal{A}$-module $F$ set $P_{I}\left(\left\{M_{i}\right\}, F\right):=$ $F \otimes_{\mathcal{A}} P_{I}^{\prime}\left(\left\{M_{i}\right\}\right) \in R$-dgm (see [Dr] C3 for the notation); if $F \in \underline{\mathcal{A}}$, this complex coincides with its namesake from 1.9.2.

Denote by $\mathcal{P}_{I}\left(\left\{M_{i}\right\}, F\right)$ the right $\mathcal{A}$-module $Q \mapsto P_{\tilde{I}}\left(\left\{M_{i}, Q\right\}, F\right)$; here $Q$ is a test object in $\mathcal{A}$ and $\tilde{I}$ is $I$ with one element added. Thus if $\mathcal{A}$ is a tensor category, then $\mathcal{P}_{I}\left(\left\{M_{i}\right\}, F\right)(Q)=F\left(\left(\otimes M_{i}\right) \otimes Q\right)$. The DG endofunctor $\mathcal{P}_{I}\left(\left\{M_{i}\right\}, \cdot\right): F \mapsto$ $\mathcal{P}_{I}\left(\left\{M_{i}\right\}, F\right)$ of $\mathcal{A}^{\mathrm{op}}$-dgm preserves quasi-isomorphisms, so it yields a triangulated (poly)functor $\mathcal{P}_{I}:\left(\mathcal{A}^{\text {tri }}\right)^{\text {op } I} \times D\left(\mathcal{A}^{\text {op }}\right) \rightarrow D\left(\mathcal{A}^{\text {op }}\right)$.

The functors $\left\{M_{i}\right\} \mapsto P_{I}\left(\left\{M_{i}\right\}, F\right), \mathcal{P}_{I}\left(\left\{M_{i}\right\}, F\right)$ extend naturally to $M_{i} \in \underline{\mathcal{A}}$ so that arbitrary direct sums of $M_{i}$ 's are transformed to direct products. The corresponding DG endofunctor $\mathcal{P}_{I}\left(\left\{M_{i}\right\}, \cdot\right)$ of $\mathcal{A}^{\mathrm{op}}$-dgm preserves quasi-isomorphisms if $M_{i} \in \underline{\mathcal{A}}^{\text {perf }}$ (see the lemma in 1.7). If $|I|=1$, we write $\mathcal{H o m}(M, F):=\mathcal{P}_{I}(\{M\}, F)$.

Remark. The endofunctor $\mathcal{P}_{I}\left(\left\{M_{i}\right\}, \cdot\right)$ of $D\left(\mathcal{A}^{\text {op }}\right)$ is right adjoint to one $F \mapsto$ $\left(\otimes M_{i}\right) \otimes F$. Thus $\mathcal{H o m}$ is an action of the tensor category $\left(\mathcal{A}^{\mathrm{tri}}\right)^{\kappa \mathrm{op}}$ on $D\left(\mathcal{A}^{\mathrm{op}}\right)$.

1.10. Presheaves. Let $T$ be an essentially small category. Assume that for every finite family $\left\{X_{s}\right\}$ of objects in $T$ the coproduct $\sqcup X_{s}$ exists, and for every morphism $Y \rightarrow X=\sqcup X_{i}$ the fiber products $Y_{s}:=Y \times_{X} X_{s}$ are representable and $\sqcup Y_{s} \stackrel{\sim}{\rightarrow} Y$. For us, a presheaf on $T$ always means a presheaf of $R$-modules compatible with finite coproducts, i.e., a functor $F: T^{\mathrm{op}} \rightarrow R$-mod such that $F\left(\sqcup X_{s}\right) \stackrel{\sim}{\rightarrow} \Pi_{s} F\left(X_{s}\right) .{ }^{12}$ Let $R[T]$ be an $R$-category whose objects are denoted by $R[X], X \in T$, and $\operatorname{Hom}(R[X], R[Y])$ is the direct limit of the directed family of free $R$-modules $\Pi_{s} R\left[\operatorname{Hom}\left(X_{s}, Y\right)\right]$ labeled by finite decompositions $\sqcup X_{s} \stackrel{\sim}{\rightarrow} X$. Then a presheaf on $T$ is the same as an $R[T]^{\mathrm{op}}$-module; we identify $R[X]$ with the corresponding representable presheaf.

${ }^{12}$ That is, a sheaf for the stupid topology whose coverings $\left\{X_{s} \rightarrow X\right\}$ are finite decompositions $\sqcup X_{s} \stackrel{\sim}{\rightarrow} X$. 
We have an abelian $R$-category $\mathcal{P S} h$ of presheaves and a DG category $C \mathcal{P} \mathcal{S} h=$ $R[T]^{\mathrm{op}}$-dgm of complexes of presheaves. Let $R[T]$ be the category of semi-free DG $R[T]^{\text {op }}$-modules (see 1.6.3). Set $\mathcal{P}:=R[T]^{\text {perf }}$, see 1.7 . Then $\underline{R[T]} \stackrel{\sim}{\rightarrow} \stackrel{\mathcal{P}}{\rightarrow}$ is the canonical DG structure on the derived category $D \mathcal{P S} h$, see (1.6.1).

Suppose $T$ is closed under finite direct products; they define a tensor structure on $T$, so $R[T]$ is an tensor $R$-category. By 1.9.2, $R[T]$ is a tensor DG category, and $\mathcal{P}$ its tensor DG subcategory. We get a tensor structure on $\underline{\mathcal{P}}^{\text {tri }}=D \mathcal{P} \mathcal{S} h$. Notice that $\mathcal{P} S h$ is a tensor abelian $R$-category in the usual way, ${ }^{13}$ and the embedding $\underline{\mathcal{P}} \hookrightarrow C \mathcal{P S} h$ is compatible with the tensor products, so the tensor structure on $\vec{D} \mathcal{P S} h$ is the standard one. The tensor DG category $\underline{\mathcal{P}}^{\text {op }}$ acts on $C \mathcal{P} \mathcal{S} h$ by the Hom action; for $X, Y \in T$ and $F \in C \mathcal{P S}$ one has $\mathcal{H o m}(R[X], F)(Y)=F(X \times Y)$.

Notation. If $U$. is a simplicial object of $T$, then $R[U$.] is a simplicial presheaf; by abuse of notation, we denote the normalized complex also by $R[U$.]. For a morphism $Y \rightarrow X$ we write $R[Y / X]:=\mathcal{C}$ one $(R[Y] \rightarrow R[X])[-1]$, for an augmented simplicial object $U$./X we have $R[U . / X]$, etc. So $R[X] \in \mathcal{P}$, and $R[U],. R[U . / X] \in \underline{\mathcal{P}}$.

1.11. Sheaves. Suppose that our $T$ is equipped with a Grothendieck topology $\mathcal{T}$ (see [SGA4] II or [D1] Arcata I 6) such that for every finite family $X_{s} \in T$ the morphisms $\left\{X_{s} \rightarrow \sqcup X_{s}\right\}$ form a covering of $\sqcup X_{s}$. We have the abelian category $\mathcal{S} h^{\mathcal{T}}$ of sheaves of $R$-modules, the exact sheafification functor $\mathcal{P} \mathcal{S} h \rightarrow \mathcal{S} h^{\mathcal{T}}, F \mapsto F_{\mathcal{T}}$, and its right adjoint $\mathcal{S} h^{\mathcal{T}} \rightarrow \mathcal{P} \mathcal{S} h$ which is fully faithful and left exact.

A complex $F$ of presheaves is said to be locally acyclic if for every $X \in T$ and $a \in H^{n} F(X)$ those $X$-objects $V / X$ that $\left.a\right|_{V} \in H^{n} F(V)$ vanishes, form a covering sieve of $X$. All such $F$ form a thick subcategory $I^{\mathcal{T}}$ of $D S h^{\mathcal{T}}$; the sheafification functor yields an equivalence $D \mathcal{P} \mathcal{S} h / I^{\mathcal{T}} \stackrel{\sim}{\rightarrow} D \mathcal{S} h^{\mathcal{T}}$.

For $X \in T$ its hypercovering (see [SGA4] V 7.3) is an augmented simplicial object of $T$ which satisfies the next condition: Let $f: \partial \Delta^{n} \times Y / Y \rightarrow U$./X be any morphism of augmented simplicial objects; here $Y \in T, n \geq 0, \Delta^{n}$ is the " $n$ simplex" simplicial set, $\partial \Delta^{n}$ its boundary. Then those $V / Y$ that $f_{V}: \partial \Delta^{n} \times V / V \rightarrow$ $U . / X$ can be extended to $\Delta^{n} \times V / V \rightarrow U$. $/ X$, form a covering sieve of $Y$.

Assume that every $X \in T$ is quasi-compact, ${ }^{14}$ has finite cohomological dimension, and each of its coverings admits a refinement $\left\{Y_{s} \rightarrow X\right\}$ such that for every morphism $Z \rightarrow X$ the fiber products $Y_{s} \times_{X} Z$ are representable.

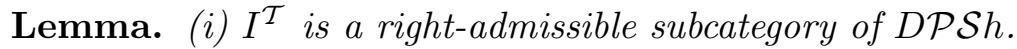

(ii) If $U . / X$ is a hypercovering of $X \in T$, then the complex $R[U . / X]$ is $\mathcal{T}$ locally acyclic. The category $I^{\mathcal{T}}$ is generated by all such complexes $R[U . / X]$.

The objects of $I^{\mathcal{T} \perp}$ are called $\mathcal{T}$-local (or simply local) complexes; by the lemma, a complex $G$ is local if and only if for every hypercovering $U$./X the map $G(X) \rightarrow$ $G(U):.=\operatorname{Hom}(R[U], G$.$) is a quasi-isomorphism. Notice that if such G$ is a single presheaf, then it is automatically a sheaf. As in 1.2 , one has the $\mathcal{T}$-localization endofunctor of $D \mathcal{P S} h, F \mapsto \mathcal{C}^{\mathcal{T}}(F):=F_{I^{\mathcal{T}} \perp}$ which identifies $D \mathcal{S} h^{\mathcal{T}}$ with $I^{\mathcal{T} \perp}$. The

${ }^{13}$ i.e., as the category of sheaves on the stupid topology from the previous footnote.

${ }^{14}$ Which means (see [SGA4] VI 1.1) that every covering of $X$ has a finite subcovering. 
t-structure picture here is from (i) of the lemma from loc. cit. As in 1.5.5, $\mathcal{P}$ yields DG structures on the subcategories $I^{\mathcal{T}}$ and $I^{\mathcal{T} \perp}$.

If $\mathcal{T}$ has enough points (see [SGA4] IV 6), then $I^{\mathcal{T}}$ is formed by complexes of sheaves whose stalks at the points are acyclic complexes. The $\mathcal{T}$-localization can be lifted to a DG endofunctor of $C \mathcal{P} \mathcal{S} h$ which assigns to a complex of presheaves its Godement resolution.

\section{$\S 2$. Correspondences and effective motives: first definitions}

This section treats the material of lectures 1, 2, 8, 23 of [MVW], $\S 6$ of [SV], and 2.1.1-2.1.3, 3.4.1 of [Vo2]. At variance with loc. cit., the constructions are carried on DG level, and the "bounded above" assumption on complexes is discarded.

2.1. Presheaves with transfer. We fix a base field $k$; let $\mathcal{S} c h=\mathcal{S} c h_{k}$ be the category of all $k$-schemes, $\mathcal{S} m=\mathcal{S} m_{k}$ be the subcategory of smooth varieties. Notice that $\mathcal{S} m$ is essentially small.

2.1.1. Using 1.10 for $T=\mathcal{S} m$, we get the abelian category $\mathcal{P} \mathcal{S} h$ of presheaves of $R$-modules on $\mathcal{S} m$, the tensor DG categories $\mathcal{P}$ and $\underline{\mathcal{P}}$, the equivalence of triangulated tensor categories

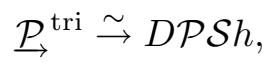

etc. Any scheme $X$ yields a presheaf $R[X]: Y \mapsto R[X](Y)=R[X(Y)]$ on $\mathcal{S} m$. As in 1.10, for $Y \rightarrow X$ we have a complex $R[Y / X]:=\mathcal{C}$ one $(R[Y] \rightarrow R[X])[-1]$, a simplicial augmented scheme $U . / X$ yields a complex $R[U . / X]$, etc.

Any presheaf $F$ on $\mathcal{S} m$ extends naturally to a presheaf on $\mathcal{S} c h$ : for a scheme $Y$ we define $F(Y)$ as $\varliminf_{\longrightarrow} F(X)$, the limit is taken over the category of pairs $(X, \nu)$ where $X \in \mathcal{S} m$ and $\nu$ is a morphism $Y \rightarrow X .{ }^{15}$ If $Y$ can be represented as the projective limit of a directed family of smooth varieties $Y_{\alpha}$ connected by affine morphisms, then $F(Y)=\varliminf F\left(Y_{\alpha}\right)$ (see [EGA IV] 8.8); in particular, the functor $F \mapsto F(Y)$ is exact. Examples of such $Y$ 's: (i) For a point $x$ of a smooth variety $X$ such is the local scheme $X_{x}:=\operatorname{Spec} \mathcal{O}_{X x}$ and its Henselization $X_{x}^{h}$; (ii) If $K / k$ is a separable extension, then such is any $Y \in \mathcal{S} m_{K}$.

2.1.2. If $X, Y$ are schemes and $Y$ is normal, then we denote by $\operatorname{Cor}(Y, X)=$ $\operatorname{Cor}_{R}(Y, X)$ the free $R$-module generated by the set of reduced irreducible subschemes $\Gamma \subset X \times Y$ such that the projection $\Gamma \rightarrow Y$ is finite and its image is an irreducible component of $Y$ (the latter condition can be replaced by that $\operatorname{dim} \Gamma=$ $\operatorname{dim} Y)$. Its elements $\gamma=\Sigma a_{i} \Gamma_{i}, a_{i} \in R$, are referred to as finite correspondences. For $Z$ normal, $Y$ smooth, we have the composition map $\operatorname{Cor}(Y, X) \otimes \mathcal{C}$ or $(Z, Y) \rightarrow$ $\operatorname{Cor}(Z, X), \gamma \otimes \gamma^{\prime} \mapsto \gamma \gamma^{\prime}:=$ the push-forward by the projection $X \times Y \times Z \rightarrow X \times Z$ of the cycle $\left(X \times \gamma^{\prime}\right) \cap(\gamma \times Z)$ on $X \times Y \times Z$. Here $\cap$ is the cycle-theoretic intersection, which is well defined due to smoothness of $Y$ (our cycles intersect properly). The composition is associative.

Remarks. (a) Informally, finite correspondences are multi-valued maps; the composition is the usual composition of multi-valued maps. (b) Any $\gamma \in \mathcal{C}$ or $(Y, X)$ is

\footnotetext{
${ }^{15}$ A morphism $(X, \nu) \rightarrow\left(X^{\prime}, \nu^{\prime}\right)$ is a morphism $f: X \rightarrow X^{\prime}$ such that $f \nu=\nu^{\prime}$.
} 
uniquely determined by its value at the generic point(s) $\gamma\left(\eta_{Y}\right)$ of $Y$, which is a zero cycle on $X_{\eta_{Y}}$. (c) By Noether's normalization lemma, every (reduced, irreducible) affine variety $\Gamma$ can be realized as a finite correspondence between some vector spaces $X, Y$. (d) The projection $\Gamma \rightarrow Y$ need not be flat: indeed, the flatness implies that $\Gamma$ is a Cohen-Macaulay scheme.

Let $\operatorname{Cor}(\mathcal{S} m)=\mathcal{C} \operatorname{or}\left(\mathcal{S} m_{k}\right)$ be the $R$-category whose objects are smooth varieties and morphisms are finite correspondences. We denote $X \in \mathcal{S} m$ considered as an object of $\operatorname{Cor}(\mathcal{S} m)$ by $R_{\mathrm{tr}}[X]$. There is a faithful embedding $R[\mathcal{S} m] \hookrightarrow \mathcal{C}$ or $(\mathcal{S} m)$, $R[X] \mapsto R_{\mathrm{tr}}[X]$, which sends a morphism $f: Y \rightarrow X$ to its graph considered as a cycle on $X \times Y$. As in 1.10, $f$ yields a complex $R_{\mathrm{tr}}[Y / X]$, etc.

We write $R_{\mathrm{tr}}$ for $R_{\mathrm{tr}}[\operatorname{Spec} k]$. For $X \in \mathcal{S} m$ the structure projection $X \rightarrow$ Spec $k$ yields the augmentation morphism $\operatorname{aug}_{X}: R_{\mathrm{tr}}[X] \rightarrow R_{\mathrm{tr}}$ and the 2-term complex $R_{\operatorname{tr}}[X /$ Spec $k]$; any 0 -cycle of degree 1 on $X$ yields a splitting of the augmentation morphism and an isomorphism $R_{\mathrm{tr}}[X / \operatorname{Spec} k] \oplus R_{\mathrm{tr}} \stackrel{\sim}{\rightarrow} R_{\mathrm{tr}}[X]$. If $Y$ is connected, then $\operatorname{Hom}\left(R_{\mathrm{tr}}[Y], R_{\mathrm{tr}}\right)=R$, so we have a morphism of $R$-modules $\operatorname{deg}: \operatorname{Cor}(Y, X) \rightarrow R, \gamma \mapsto \operatorname{aug}_{X} \gamma$. One has $\operatorname{deg}\left(\gamma \gamma^{\prime}\right)=\operatorname{deg}(\gamma) \operatorname{deg}\left(\gamma^{\prime}\right)$.

One refers to $\mathcal{C}$ or $(\mathcal{S} m)^{\text {op }}$-modules as presheaves with transfers (on $\mathcal{S} m_{k}$ ); they form an abelian category $\mathcal{P} \mathcal{S} h_{\mathrm{tr}}$. The evident forgetful functor $o: \mathcal{P S} h_{\mathrm{tr}} \rightarrow \mathcal{P} \mathcal{S} h$ is exact and faithful, so presheaves with transfers can be considered as presheaves with an extra structure (that of transfer). Notice that any scheme $X \in \mathcal{S}$ ch yields a presheaf with transfers $Y \mapsto \mathcal{C}$ or $(Y, X)$ which we denote by $R_{\mathrm{tr}}[X]$. We have defined a functor $\mathcal{S} c h \rightarrow \mathcal{P S} h_{\mathrm{tr}}$.

Exercises. (i) Let $Y / X$ be a Galois étale covering of smooth varieties with the Galois group $G$, and $F$ be a presheaf with transfers such that the multiplication by $|G|$ on $F$ is invertible. Then $F(X) \stackrel{\sim}{\rightarrow} F(Y)^{G}$.

(ii) If $K / k$ be a separable extension, then any presheaf with transfers on $\mathcal{S} m_{k}$ defines naturally a presheaf with transfers on $\mathcal{S} m_{K}$ (as a mere presheaf it was defined in 2.1.1).

2.1.3. The next approach to $\mathcal{C}$ or is sometimes convenient. Suppose $R=\mathbb{Z}$. A correspondence $\gamma=\Sigma a_{i} \Gamma_{i} \in \mathcal{C} \operatorname{Cor}(Y, X)$ is said to be effective if all $a_{i} \geq 0$, so $\operatorname{Cor}(Y, X)$ is the group completion of the commutative monoid $\operatorname{Cor}(Y, X)^{\mathrm{eff}}$ of effective correspondences. The composition of effective correspondences is effective.

Suppose $X$ is quasi-projective; then $\operatorname{Sym}^{\prime} X=\underset{n \geq 0}{\sqcup} \operatorname{Sym}^{n} X$ is a universal commutative monoid scheme generated by $X$. Notice that if $Y$ is another quasi-projective scheme, then $(\operatorname{Sym} X)(Y)$ is the same as the set of morphisms of monoid schemes $\operatorname{Sym} Y Y \rightarrow \operatorname{Sym} \cdot X$.

Proposition. There is a natural morphism of monoids

$$
\sigma: \mathcal{C} \text { or }(Y, X)^{\mathrm{eff}} \rightarrow(\operatorname{Sym} X)(Y)
$$

If $p=$ char $k$ is zero, this is an isomorphism; if $p$ is finite, then $\sigma$ becomes an isomorphism after inverting $p$ (so $\sigma$ is injective, for $\mathcal{C}$ or has no torsion). The map $\sigma$ is compatible with composition (for quasi-projective $Y$ ).

Proof. We can assume that $Y$ is irreducible. 
(a) We define $\sigma$ on the base of $\mathcal{C} \operatorname{or}(Y, X)^{\text {eff }}$ formed by irreducible correspondences $\Gamma \subset X \times Y$. Let $n$ be the degree of $\Gamma$. Write $\Gamma=\operatorname{Spec} \mathcal{A}_{Y}$ where $\mathcal{A}_{Y}$ is a coherent

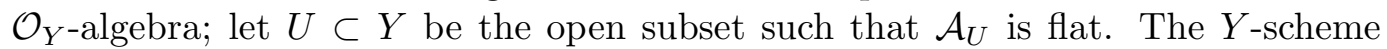
$\operatorname{Sym}^{n}(\Gamma / Y)=\operatorname{Spec}\left(\mathcal{A}_{Y}^{\otimes n}\right)^{\Sigma_{n}}$ has a natural section $s_{\Gamma}$ over $U$ : for $f \in \mathcal{A}_{U}$ the function $s_{\Gamma}^{*} f^{\otimes n} \in \mathcal{O}_{U}$ is the determinant of the multiplication by $f$ action on the $\mathcal{O}_{U}$-module $\mathcal{A}_{U}$. Since $Y$ is normal, $s_{\Gamma}$ extends uniquely to a section of $\operatorname{Sym}^{n}(\Gamma / Y)$ over $Y$, and (2.1.2) assigns to $\Gamma$ the composition $Y \stackrel{s_{\Gamma}}{\longrightarrow} \operatorname{Sym}^{n}(\Gamma / Y) \rightarrow \operatorname{Sym}^{n} X$.

Remark. For $Y$ regular, we have an invertible $\mathcal{O}_{Y}$-module $\operatorname{det}_{\mathcal{O}_{Y}} \mathcal{A}_{Y}$; the group $\operatorname{Aut}_{\mathcal{O}_{Y}}\left(\mathcal{A}_{Y}\right)$ acts on it by transport of structure. For $f \in \mathcal{A}_{Y}^{\times}$the function $s_{\Gamma}^{*} f^{\otimes n}$ is the action of the multiplication by $f$ automorphism of $\mathcal{A}_{Y}$ on $\operatorname{det}_{\mathcal{O}_{Y}} \mathcal{A}_{Y}$.

(b) Let us show that $\gamma_{V} \in \mathcal{C} \operatorname{Cor}(V, X)^{\text {eff }}, V \subset Y$ an open subset, comes from $\operatorname{Cor}(Y, X)^{\text {eff }}$ if (and only if) the map $\sigma\left(\gamma_{V}\right): V \rightarrow \operatorname{Sym}^{n} X, n=\operatorname{deg}\left(\gamma_{V}\right)$, extends to $Y$. Let $Z_{V} \subset X \times V$ be the support of the cycle $\gamma_{V}, Z$ its closure in $X \times Y$; then $\gamma_{V}$ extends to $Y$ if (and only if) $Z$ is finite over $Y$. Suppose $\sigma\left(\gamma_{V}\right)$ extends to $f: Y \rightarrow \operatorname{Sym}^{n} X$. Consider $X^{n}$ as a finite $\operatorname{Sym}^{n} X$-scheme; let $\tilde{Z}:=X^{n} \underset{\operatorname{Sym}^{n} X}{\times} Y \subset$ $X^{n} \times Y$ be its $f$-pull-back, and $Z^{\prime} \subset X \times Y$ be the image of $\tilde{Z}$ by any of the projections $X^{n} \rightarrow X$. Then $Z^{\prime}$ is finite over $Y$ (for such is $\tilde{Z}$ ) and $Z^{\prime} \supset Z_{V}$, so $Z$ is finite over $Y$, q.e.d.

(c) By (b), it suffices to check the isomorphism assertion at the generic point $\eta$ of $Y$. Replacing $k$ by $\eta, X$ by $X_{\eta}$, we can assume that $Y=\operatorname{Spec} k$ (so $\operatorname{Cor}(Y, X)$ is the group $Z_{0}(X)$ of zero cycles on $\left.X\right)$. It is clear that $\sigma$ is an isomorphism if $k$ is algebraically closed; by elementary Galois theory same is true if $k$ is perfect. The case of arbitrary $k$ reduces to its perfect closure, since for any purely inseparable finite extension $k^{\prime} / k$ both compositions of the usual maps $Z_{0}(X) \rightleftarrows Z_{0}\left(X_{k^{\prime}}\right)$ are multiplications by $\left[k^{\prime}: k\right]$ (and $\left(\operatorname{Sym}^{\prime} X\right)(k) \rightarrow\left(\mathrm{Sym}^{\prime} X\right)\left(k^{\prime}\right)$ is injective), q.e.d.

(d) Suppose $Z$ is normal, $Y$ is quasi-projective and smooth; let us check that for $\gamma \in \mathcal{C}$ or $(Y, X)^{\text {eff }}, \gamma^{\prime} \in \mathcal{C}$ or $(Z, Y)^{\text {eff }}$ one has $\sigma\left(\gamma \gamma^{\prime}\right)=\sigma(\gamma) \sigma\left(\gamma^{\prime}\right)$, where $\sigma(\gamma)$ is understood as a morphism of monoid schemes $\operatorname{Sym}^{\prime} Y \rightarrow \operatorname{Sym}^{\circ} X$. It suffices to check the equality at a generic point of $Z$; by the base change to the generic point(s) of $\gamma^{\prime}$, we are reduced to the case $Z=\operatorname{Spec} k, \gamma^{\prime}=y \in Y(k)$. One can assume that $\gamma$ is as in (a), and, replacing $X$ by an affine neighborhood of $\gamma(y)$, that $X$ is affine, $X=\operatorname{Spec} B$. Then $z:=\sigma\left(\gamma \gamma^{\prime}\right)$ and $z^{\prime}:=\sigma(\gamma) \sigma\left(\gamma^{\prime}\right)$ are $k$-points of $\operatorname{Sym}^{n} X=\operatorname{Spec}\left(B^{\otimes n}\right)^{\Sigma_{n}}$. The latter algebra is generated by elements $b^{\otimes n}$ with $b \in B$ invertible at $\gamma(y)$. It remains to show that $b^{\otimes n}(z)=b^{\otimes n}\left(z^{\prime}\right)$. If $\gamma(y)=\Sigma n_{i} x_{i}$, then $b^{\otimes n}(z)=\prod \mathrm{Nm}_{k\left(x_{i}\right) / k} b\left(x_{i}\right)^{n_{i}}$ where $\mathrm{Nm}_{k\left(x_{i}\right) / k}: k\left(x_{i}\right) \rightarrow k$ is the norm map. By Remark in (a), $b\left(z^{\prime}\right)$ is the determinant of the multiplication by $b$ action on the derived fiber $L i_{y}^{*} \mathcal{A}_{Y}$ of $\mathcal{A}_{Y}$ at $y$. The latter is a complex of $B$-modules supported at $\left\{x_{i}\right\}$ with the Euler characteristic at $x_{i}$ equal to $n_{i}$, and we are done.

As an application, let us show that for any commutative algebraic group $G$ over $k$ the presheaf $X \mapsto G(X)$ admits a natural transfer structure. Let $\pi:$ Sym $G \rightarrow G$ be the morphism of monoid schemes which equals $\operatorname{id}_{G}$ on $\operatorname{Sym}^{1} G$. Now for an effective $\gamma \in \mathcal{C}$ or $(Y, X)$ the map $\gamma^{*}: G(X) \rightarrow G(Y)$ sends $a \in G(X)$ to the composition $Y \stackrel{a \gamma}{\longrightarrow} \operatorname{Sym}^{\cdot} G \stackrel{\pi}{\rightarrow} G$. In particular, the sheaf $\mathcal{O}^{\times}$of invertible functions admits a natural transfer structure (given by the norm).

Remark. Consider an additive category whose objects are quasi-projective schemes 
and morphisms are group completions of the morphisms between the monoid schemes Sym $X$. The proposition shows that the category of smooth quasi-projective varieties and finite correspondences embeds into this category (and the embedding is fully faithful if $\operatorname{char} k=0$ ).

2.1.4. Consider the DG category of complexes $C \mathcal{P} \mathcal{S} h_{\mathrm{tr}}$, which is the same as the DG category $\mathcal{C}$ or $(\mathcal{S} m)^{\mathrm{op}}$-dgm of DG $\mathcal{C}$ or $(\mathcal{S} m)^{\mathrm{op}}$-modules; it contains the full DG category $\mathcal{P}_{\text {tr }}$ of semi-free $\mathcal{C}$ or $(\mathcal{S} m)^{\mathrm{op}}$-modules (see 1.6). As in Remark (ii) in 1.7 , let $\mathcal{P}_{\text {tr }}:=\left(\mathcal{\mathcal { P }}_{\text {tr }}\right)^{\text {perf }}$ be the homotopy idempotent completion of $\mathcal{C}$ or $(\mathcal{S} m)^{\text {pretr }}$, so $\mathcal{P}_{\mathrm{tr}}^{\mathrm{tri}}=\left(\mathcal{C} \text { or }(\mathcal{S} m)^{\mathrm{tri}}\right)^{\kappa}$. By $(1.6 .1), \underline{\mathcal{P}}_{\mathrm{tr}}$ is a DG structure on $D \mathcal{P} \mathcal{S} h_{\mathrm{tr}}$ :

$$
\stackrel{\mathcal{P}_{\mathrm{tr}}^{\mathrm{tri}}}{\longrightarrow} \stackrel{\sim}{\rightarrow} D \mathcal{P} \mathcal{S} h_{\mathrm{tr}}
$$

Remarks. (i) The embedding $R[\mathcal{S} m] \hookrightarrow \mathcal{C}$ or $(\mathcal{S} m), R[X] \mapsto R_{\operatorname{tr}}[X]$, yields DG functors $\mathcal{P} \rightarrow \mathcal{P}_{\mathrm{tr}}, \underline{\mathcal{P}} \rightarrow \underset{\mathcal{P}_{\mathrm{tr}}}{ }$. The corresponding functor $\mathcal{\mathcal { P }}^{\text {tri }} \rightarrow \underset{\mathcal{P}_{\mathrm{tr}}^{\mathrm{tri}}}{\longrightarrow}$ is left adjoint, via identifications (2.1.1) and (2.1.3), to the forgetful functor $o: D \mathcal{P} \mathcal{S} h_{\mathrm{tr}} \rightarrow D \mathcal{P} \mathcal{S}$, so we have the adjoint pair

$$
D \mathcal{P S h} \rightleftarrows D \mathcal{P S} h_{\mathrm{tr}}
$$

(ii) If $K / k$ is any extension, then the base change functor $\mathcal{S} m_{k} \rightarrow \mathcal{S} m_{K}, X \mapsto$ $X_{K}$, extends naturally to $\operatorname{Cor}(\mathcal{S} m)$ and the DG categories $\underset{\mathcal{P}_{\text {tr }}}{ }, \mathcal{P}_{\text {tr }}$. If $K / k$ is separable, then we have a similar exact functor for the categories of presheaves with transfers (see Exercise (ii) in 2.1.2), and (2.1.3) is compatible with these functors.

Voevodsky's notation for $\mathcal{P} \mathcal{S} h_{\mathrm{tr}}$ is $\operatorname{PreShv}(\operatorname{SmCor}(k))$ in [Vo2], and PST in [MVW].

2.2. The tensor product and inner Hom; the Tate twist. The direct product of smooth varieties defines on $\operatorname{Cor}(\mathcal{S} m)$ a tensor category structure, $R_{\mathrm{tr}}[X] \otimes R_{\mathrm{tr}}[Y]=R_{\mathrm{tr}}[X \times Y]$, with the unit object $R_{\mathrm{tr}}$. Thus $\mathcal{P}_{\mathrm{tr}}$ and $\underset{\mathcal{P}_{\mathrm{tr}}}{\longrightarrow}$ are DG tensor categories (see 1.9.2), and $D \mathcal{P} \mathcal{S} h_{\mathrm{tr}}$ is a tensor triangulated category (via (2.1.3)). The tensor product is right t-exact for the evident t-structure on $D \mathcal{P} \mathcal{S} h_{\mathrm{tr}}$.

As in 1.9.3, the DG tensor category $\mathcal{P}_{\mathrm{tr}}^{\text {op }}$ acts naturally on $C \mathcal{P} \mathcal{S} h_{\mathrm{tr}}$. The action of $P \in \mathcal{P}_{\mathrm{tr}}$ is denoted by $F \mapsto \mathcal{H o m}(P, F)$, so for $P=R_{\mathrm{tr}}[X]$ one has $\mathcal{H o m}\left(R_{\mathrm{tr}}[X], F\right)(Y)=F(X \times Y)$. Passing to the homotopy categories, we get the action of the tensor triangulated category $\mathcal{P}_{\mathrm{tr}}^{\text {optri }}$ on $D \mathcal{P} \mathcal{S} h_{\mathrm{tr}}$. The endofunctors $F \mapsto P \otimes F$ and $F \mapsto \mathcal{H o m}(P, F)$ of $D \mathcal{P} \mathcal{S} h_{\mathrm{tr}}=\mathcal{\mathcal { P }}_{\mathrm{tr}}^{\text {tri }}$ are adjoint.

By 1.10, the above picture remains valid if we replace $\mathcal{C}$ or $(\mathcal{S} m)$ by $R[\mathcal{S} m]$. Since $R[\mathcal{S} m] \hookrightarrow \operatorname{Cor}(\mathcal{S} m)$ is a tensor functor, $\mathcal{P} \rightarrow \mathcal{P}_{\mathrm{tr}}, \underline{\mathcal{P}} \rightarrow \underline{\mathcal{P}_{\mathrm{tr}}}$, and $D \mathcal{P} \mathcal{S} h \rightarrow D \mathcal{P} h_{\mathrm{tr}}$ are also tensor functors. The forgetful functor $o: C \mathcal{P} \mathcal{S} h_{\mathrm{tr}} \rightarrow C \mathcal{P S} h$ is compatible with the $\mathcal{P}^{\text {op }}$-action.

Remark. Since $o: D \mathcal{P} \mathcal{S} h_{\mathrm{tr}} \rightarrow D \mathcal{P S} h$ is right adjoint to a tensor functor $\underline{\mathcal{P}}^{\text {tri }} \rightarrow$ $\overrightarrow{\mathcal{P}}_{\mathrm{tr}}^{\text {tri }}$, it acts naturally on polylinear operations (i.e., in the terminology of [BD] 1.1.5, $o$ is a pseudo-tensor functor). Explicitly, for a finite collection of objects $F_{i}, G \in D \mathcal{P} \mathcal{S} h_{\text {tr }}$ one has a canonical map $\operatorname{Hom}\left(\otimes F_{i}, G\right) \rightarrow \operatorname{Hom}\left(\otimes o\left(F_{i}\right), o(G)\right)$ 
which assigns to $\phi: \otimes F_{i} \rightarrow G$ the composition $\otimes o\left(F_{i}\right) \rightarrow o\left(\otimes F_{i}\right) \stackrel{o(\phi)}{\longrightarrow} o(G)$, where the first arrow comes from the evident maps $\otimes \operatorname{Cor}\left(Z, X_{i}\right) \rightarrow \operatorname{Cor}\left(Z, \prod X_{i}\right)$ (the latter are not isomorphisms in general, so $o$ is not a tensor functor). Therefore $o$ transforms any kind of algebras in $D \mathcal{P} \mathcal{S} h_{\mathrm{tr}}$ to algebras of similar kind in $D \mathcal{P} \mathcal{S} h$.

Set $R(1):=R\left[\mathbb{G}_{m} /\right.$ Spec $\left.k\right][-1] \in \mathcal{P}$ where $\mathbb{G}_{m}:=\mathbb{A}^{1} \backslash\{0\} .{ }^{16}$ We denote the corresponding object of $\mathcal{P}_{\mathrm{tr}}$ by $R_{\mathrm{tr}}(1)$. As in 2.1.1, every $a \in \mathbb{G}_{m}(k)$ yields a morphism $R \rightarrow R\left[\mathbb{G}_{m}\right]$ so that $R \oplus R(1)[1] \stackrel{\sim}{\rightarrow} R\left[\mathbb{G}_{m}\right]$; the standard choice is $a=1$. The tensor product with $R(1)$ or $R_{\mathrm{tr}}(1)$ in $\mathcal{P}, \mathcal{P}_{\mathrm{tr}}$ and related tensor categories is denoted by $F \mapsto F(1)$; this is the Tate twist endofunctor.

For $F \in \mathcal{P S} h$ we define a presheaf $F_{-1}$ by formula $F_{-1}(X):=F\left(\left(X \times \mathbb{G}_{m}\right) / X\right)=$ $\operatorname{Coker}\left(F(X) \rightarrow F\left(X \times \mathbb{G}_{m}\right)\right)$ where the arrow comes from the projection $X \times \mathbb{G}_{m} \rightarrow$ $X$. Same construction works in the case of presheaves with transfers. We can also pass to complexes; one has $F_{-1}=\mathcal{H o m}(R(1)[1], F)$. The endofunctor $F \mapsto F_{-1}[1]$ of $D \mathcal{P} \mathcal{S} h_{\mathrm{tr}}$ is right adjoint to the Tate twist.

The iterations of the above functors are denoted by $F \mapsto F(n), F_{-n}, n \in \mathbb{Z}_{\geq 0}$.

2.3. The DG category of effective motives. Consider the following two types of complexes in $\mathcal{C}$ or $(\mathcal{S} m)$ :

$(\Delta)$ The 2-term complexes $R_{\mathrm{tr}}\left[\mathbb{A}^{1} \times X\right] \rightarrow R_{\mathrm{tr}}[X]$, the differential comes from the projection $\mathbb{A}^{1} \times X \rightarrow X, X \in \mathcal{S} m$.

$\left(\mathrm{MV}_{\mathrm{Zar}}\right)$ The 3 -term Mayer-Vietoris ones $R_{\mathrm{tr}}[U \cap V] \rightarrow R_{\mathrm{tr}}[U] \oplus R_{\mathrm{tr}}[V] \rightarrow R_{\mathrm{tr}}[X]$. Here $U, V \subset X$ are Zariski open subsets such that $U \cup V=X$, and the differentials are, respectively, the difference and the sum of the embedding morphisms.

Let $\mathcal{I}_{\mathrm{tr}}^{\Delta}, \mathcal{I}_{\mathrm{tr}}^{\text {Zar }}, \mathcal{I}_{\mathrm{tr}}^{\Delta \text { Zar }} \subset \mathcal{P}_{\mathrm{tr}}$ be the homotopy idempotent completions of the full pretriangulated DG subcategories strongly generated by the objects of the corresponding types (see 1.6.2). Set (see 1.8)

$$
\mathcal{D}_{\mathcal{M}}^{\text {eff }}=\mathcal{D}_{\mathcal{M} / k}^{\text {eff }}:=\mathcal{P}_{\text {tr }}{ }^{\kappa} \mathcal{I}_{\text {tr }}^{\Delta \text { Zar }} .
$$

This is the DG category of effective geometric motives. The homotopy object of $\mathcal{D}_{\mathcal{M}}^{\text {eff }}$ that corresponds to $R_{\mathrm{tr}}[X]$ is denoted by $M(X)$; this is the motive of $X$.

Passing to the "infinite" categories, we see, by (1.8.1), that

$$
\stackrel{\mathcal{D}_{\mathcal{M}}^{\mathrm{eff}}}{\longrightarrow} \stackrel{\sim}{\rightarrow} \underline{\mathcal{P}_{\mathrm{tr}}} / \underline{\mathcal{I}}_{\mathrm{tr}}^{\Delta \mathrm{Zar}}
$$

This is the DG category of effective motives.

Consider the corresponding homotopy categories. We use identification (2.1.3). Set $I_{\mathrm{tr}}^{\mathrm{Zar}}:=\stackrel{\mathcal{I}_{\mathrm{tr}}^{\mathrm{Zar}} \text { tri }}{\longrightarrow}$, etc; these are right-admissible subcategories of $D \mathcal{P} \mathcal{S} h_{\mathrm{tr}}$, and

$$
\begin{aligned}
& \underline{\mathcal{D}}_{\mathcal{M}}^{\text {eff tri }} \stackrel{\sim}{\rightarrow} D \mathcal{P} \mathcal{S} h_{\mathrm{tr}} / I_{\mathrm{tr}}^{\Delta \mathrm{Zar}} \stackrel{\sim}{\leftarrow}\left(I_{\mathrm{tr}}^{\Delta \text { Zar }}\right)^{\perp}, \\
& \mathcal{D}_{\mathcal{M}}^{\text {eff tri }} \stackrel{\sim}{\rightarrow}\left(D \mathcal{P} \mathcal{S} h_{\mathrm{tr}} / I_{\text {tr }}^{\Delta \text { Zar }}\right)^{\text {perf }} .
\end{aligned}
$$

\footnotetext{
${ }^{16}$ At the moment, we do not care about the group structure on $\mathbb{G}_{m}$. Our notation is not consistent with that of $[\mathrm{MVW}]$ and [Vo2]: in loc. cit., $\mathbb{G}_{m}$ denotes the pointed variety $\left(\mathbb{A}^{1} \backslash\{0\}, 1\right)$.
} 
The right $I_{\mathrm{tr}}^{\Delta \text { Zar }}$-localization endofunctor $\mathcal{C}^{\mathcal{M}}: D \mathcal{P} \mathcal{S} h_{\mathrm{tr}} \rightarrow D \mathcal{P} \mathcal{S} h_{\mathrm{tr}}$ is called the motivic localization; it will be studied in the next two sections.

Notice that the tensor product by any object of $\mathcal{C}$ or $(\mathcal{S} m)$ preserves the complexes of type $(\Delta)$ and of type (Zar). As in 1.9 , the above $\mathcal{I}_{\text {tr }}^{\text {? }}$ 's are ideals in $\mathcal{P}_{\text {tr }}$, and $\mathcal{D}_{\mathcal{M}}^{\text {eff }}$ is a homotopy tensor DG category with the unit object $R_{\mathcal{M}}:=M(\operatorname{Spec} k)$. Same is true for the "infinite" categories.

The image in $\mathcal{D}_{\mathcal{M}}^{\mathrm{eff}}$ of $R_{\mathrm{tr}}(1) \in \mathcal{P}_{\mathrm{tr}}$ is the Tate motive $R_{\mathcal{M}}(1)$.

Voevodsky's notation for $\mathcal{D}_{\mathcal{M}}^{\text {eff tri }}=\mathcal{D}_{\mathcal{M} / k}^{\text {eff tri }}$ is $D M_{g m}^{\text {eff }}(k)$ in [Vo2].

Remarks. (i) In $\S 4$ we will see how to perform the motivic localization explicitly if the base field $k$ is perfect. It is not clear if the above definition is reasonable for non perfect $k$ (see e.g. Remark (b) in 4.4).

(ii) The embedding $\underline{\mathcal{D}}_{\mathcal{M}}^{\text {eff }}$ tri $\stackrel{\sim}{\rightarrow}\left(I_{\mathrm{tr}}^{\Delta \text { Zar }}\right)^{\perp} \subset D \mathcal{P} \mathcal{S} h_{\text {tr }}$ is a pseudo-tensor functor (as the right adjoint to a tensor functor, cf. Remark in 2.2). So $\mathcal{C}^{\mathcal{M}}: D \mathcal{P} \mathcal{S} h_{\mathrm{tr}} \rightarrow$ $D \mathcal{P S} h_{\mathrm{tr}}$ is a pseudo-tensor functor as well.

2.4. The Artin motives. Let $\mathcal{S} m_{0}$ be the category of smooth zero-dimensional varieties. The embedding $\mathcal{S} m_{0} \hookrightarrow \mathcal{S} m$ admits a left adjoint functor $\pi_{0}: \mathcal{S} m \rightarrow$ $\mathcal{S} m_{0}$, where $\pi_{0}(X)$ is the spectrum of the integral closure of $k$ in $\mathcal{O}(X)$. The corresponding full subcategory $\operatorname{Cor}\left(\mathcal{S} m_{0}\right) \subset \mathcal{C}$ or $(\mathcal{S} m)$ is an additive $R$-category, and $\pi_{0}$ extends naturally to an $R$-linear functor $\pi_{0}: \mathcal{C}$ or $(\mathcal{S} m) \rightarrow \mathcal{C}$ or $\left(\mathcal{S} m_{0}\right)$ left adjoint to the embedding.

Pick a separable closure $\bar{k} / k$ of $k$; let $G$ be the Galois group. By Galois theory, the functor $X \mapsto X(\bar{k})$ identifies $\mathcal{S} m_{0}$ with the category of finite $G$-sets. This implies easily that the functor $R_{\mathrm{tr}}[X] \mapsto R[X(\bar{k})]$ is a fully faithful embedding of $\operatorname{Cor}\left(\mathcal{S} m_{0}\right)$ into the category of $R[G]$-modules; its essential image is the category $R[G]$-prm of permutative $R[G]$-modules.

The DG category $\mathcal{A} r=\mathcal{A} r_{k}$ of Artin motives is defined as the homotopy idempotent completion of $\mathcal{C}$ or $\left(\mathcal{S} m_{0}\right)$ inside $\mathcal{P}_{\operatorname{tr}}$ (see 1.6.2,1.7). So the triangulated category $\mathcal{A} r^{\text {tri }}$ is the idempotent completion of the homotopy category of finite complexes of objects of $\mathcal{C}$ or $\left(\mathcal{S} m_{0}\right)$, i.e., $\mathcal{A} r^{\text {tri }}=\left(K^{b} R[G] \text {-prm }\right)^{\kappa}$. The Artin motives form a full subcategory in $\mathcal{D}_{\mathcal{M}}^{\text {eff tri. }}$

Lemma. The functor $\pi_{0}^{\text {tri }}$ factors through a triangulated functor $\mathcal{D}_{\mathcal{M}}^{\mathrm{eff} \text { tri }} \rightarrow \mathcal{A} r^{\text {tri }}$, which is left adjoint to the composition $\mathcal{A} r^{\text {tri }} \hookrightarrow\left(\mathcal{C o r}(\mathcal{S} m)^{\text {tri }}\right)^{\kappa} \rightarrow \mathcal{D}_{\mathcal{M}}^{\text {effi }}$. The latter composition is a fully faithful embedding $\mathcal{A} r^{\text {tri }} \hookrightarrow \mathcal{D}_{\mathcal{M}}^{\text {eff tri }}$.

Proof. To see that $\pi_{0}^{\text {tri }}: \mathcal{C}$ or $(\mathcal{S} m)^{\text {tri }} \rightarrow \mathcal{A} r^{\text {tri }}$ factors through $\mathcal{D}_{\mathcal{M}}^{\text {eff tri }}$, we need to check that $\pi_{0}^{\text {tri }}$ kills complexes $(\Delta)$ and $\left(\mathrm{MV}_{\mathrm{Zar}}\right)$ from 2.3. This is evident since $\pi_{0}\left(\mathbb{A}^{1} \times X\right)=\pi_{0}(X)$ and $\pi_{0}(U.) \rightarrow \pi_{0}(X)$ is a homotopy equivalence. The rest is immediate.

Remark. Let $G$ be any (pro)finite group; suppose that $R$ is noetherian regular. Then, according to $[\mathrm{R}]$, the evident functor $K^{b} R[G]$-prm $\rightarrow D^{b} R[G]$-mod (the bounded derived category of finitely generated $R[G]$-modules) yields an equivalence $\left(K^{b} R[G] \text {-prm } / I\right)^{\kappa} \stackrel{\sim}{\rightarrow} D^{b} R[G]$-mod; here $I$ is the thick subcategory of acyclic 
complexes of permutative modules.

\section{$\S 3$. Premotives and the $\mathbb{A}^{1}$-homotopy localization}

In this section we consider the $\mathbb{A}^{1}$-homotopy localization procedure used in the definition of the category of motives more thoroughly. The material corresponds to lectures 4 and 9 of [MVW] and [Vo3]. The constructions (if not the terminology) of 3.1 go back to [S]. The principal result is theorem 3.3 (Voevodsky's cancellation theorem); its proof is a variant of Voevodsky's original one [Vo3]. The construction in 3.4.1 is used to define the residue maps in 4.6; the theme of 3.4.2 is developed in 5.1 .4 .

3.1. The $\mathbb{A}^{1}$-homotopy localization. 3.1.1. Let $I^{\Delta} \subset D \mathcal{P S} h$ be the triangulated subcategory generated by all complexes of type $R\left[\mathbb{A}^{1} \times X / X\right]$ where $X \in \mathcal{S} m$ and $\mathbb{A}^{1} \times X \rightarrow X$ is the projection. Its objects are $\mathbb{A}^{1}$-homotopy contractible complexes. One has the corresponding subcategory $I^{\Delta \perp} \subset D \mathcal{P} S h$ of $\mathbb{A}^{1}$-homotopy invariant, or $\mathbb{A}^{1}$-homotopy local, complexes. A complex of presheaves $F$ lies in $I^{\Delta \perp}$ if and only if for every $X$ the morphism $F(X) \rightarrow F\left(\mathbb{A}^{1} \times X\right)$ is a quasi-isomorphism, i.e., the morphism $F \rightarrow \mathcal{H o m}\left(R\left[\mathbb{A}^{1}\right], F\right)$ is a quasi-isomorphism in $D \mathcal{P} \mathcal{S} h . F$ is $\mathbb{A}^{1}$-homotopy invariant if and only if all the cohomology $H^{a} F$ are $\mathbb{A}^{1}$-homotopy invariant. Thus $I^{\Delta \perp}$ carries a non-degenerate t-structure whose core is the abelian category $\mathcal{P} \mathcal{S} h^{\Delta}$ of $\mathbb{A}^{1}$-homotopy invariant presheaves.

Exercise. $F \in D \mathcal{P S h}$ is $\mathbb{A}^{1}$-homotopy invariant iff the pull-back maps $i_{0}^{*}, i_{1}^{*}$ : $\mathcal{H o m}\left(R\left[\mathbb{A}^{1}\right], F\right) \rightrightarrows F$ for the embeddings $i_{a}: \operatorname{Spec} k \hookrightarrow \mathbb{A}^{1}, \cdot \mapsto a \in \mathbb{A}^{1}$, coincide.

Our $I^{\Delta}$ is right admissible by 1.4 .2 , so $I^{\Delta \perp} \stackrel{\sim}{\rightarrow} D \mathcal{P} \mathcal{S} h / I^{\Delta}$, and the t-structure picture fits into the format of part (ii) of the lemma in 1.2 .

In fact, the $I^{\Delta}$-localization functor lifts to a DG endofunctor $\mathcal{C}^{\Delta}$ of $C \mathcal{P} \mathcal{S} h$ defined as follows. Let $\Delta$ be the standard cosimplicial scheme of the "simplices" $\Delta^{n}:=$ $\left\{\left(t_{0}, \ldots, t_{n}\right) \in \mathbb{A}^{n+1}: \sum t_{i}=1\right\}$. A presheaf $F$ yields then a simplicial presheaf $\mathcal{H o m}\left(R\left[\Delta^{\cdot}\right], F\right): X \mapsto F\left(X \times \Delta^{*}\right)$; let $C^{\Delta}(F)$ be its normalized complex. Explicitly, $C^{\Delta}(F)^{n}(X):=\left\{f \in F\left(X \times \Delta^{n}\right): \partial_{1}(f)=\ldots=\partial_{n}(f)=0\right\}$, the differential is $f \mapsto$ $\partial_{0}(f)$. For $F \in C \mathcal{P S} h$ we denote by $C^{\Delta}(F)$ the total complex of the corresponding bicomplex $\left(\right.$ so $\mathcal{C}^{\Delta}(F)^{n}(X)=\sum_{a-b=n} F^{a}\left(X \times \Delta^{b}\right)$ ). In the notation of 1.9 and 2.2, one has $\mathcal{C}^{\Delta}(F)=\varliminf \mathcal{H o m}\left(\sigma_{\leq n} R\left[\Delta^{\cdot}\right]^{\text {nor }}, F\right)$; here $R\left[\Delta^{\cdot}\right]$ is a cosimplicial object in $R[\mathcal{S} m], R\left[\Delta^{\cdot}\right]^{\text {nor }}$ its normalized complex, and $\sigma_{\leq n} R\left[\Delta^{\cdot}\right]^{\text {nor }}$ are its stupid truncations (which are finite complexes). A natural embedding $F \hookrightarrow \mathcal{C}^{\Delta}(F)$ identifies $F$ with the component of the $b$-degree 0 . Since $\mathcal{C}^{\Delta}$ preserves quasi-isomorphisms, it yields an endofunctor on $D \mathcal{P S} h$. The latter equals the $\mathbb{A}^{1}$-homotopy localization:

Proposition. One has $\mathcal{C}^{\Delta}(F) \in I^{\Delta \perp}, \mathcal{C}^{\Delta}(F) / F \in I^{\Delta}$.

Proof. We show that for a presheaf $F$ the complex $\mathcal{C}^{\Delta}(F)$ is $\mathbb{A}^{1}$-homotopy invariant, leaving the rest for the reader. It suffices to construct a simplicial homotopy $h$. between the morphisms of simplicial $R$-modules $i_{0}^{*}, i_{1}^{*}: F\left(\mathbb{A}^{1} \times X \times \Delta^{*}\right) \rightrightarrows F\left(X \times \Delta^{*}\right)$. Let $T$. be the simplicial interval, i.e., the simplicial set with $T_{n}=$ the set of monotone maps $a:[0, n] \rightarrow[0,1]$. Our $h$. is comprised by maps $h_{n}: F\left(\mathbb{A}^{1} \times X \times \Delta^{n}\right) \otimes$ $R\left[T_{n}\right] \rightarrow F\left(X \times \Delta^{n}\right)$, i.e., by those $h_{n}^{a}: F\left(\mathbb{A}^{1} \times X \times \Delta^{n}\right) \rightarrow F\left(X \times \Delta^{n}\right), a \in T_{n}$, 
$h_{n}(f \otimes a)=h_{n}^{a}(f)$. We define $h_{n}^{a}$ as the pull-back for a map $X \times \Delta^{n} \rightarrow \mathbb{A}^{1} \times X \times \Delta^{n}$, $\left(x,\left(t_{0}, \ldots, t_{n}\right)\right) \mapsto\left(\sum a(i) t_{i}, x,\left(t_{0}, \ldots, t_{n}\right)\right)$.

Exercise. Suppose a presheaf $F$ admits a natural map $\kappa: F(X) \rightarrow F\left(\mathbb{A}^{1} \times X\right)$ which preserves the zero section ( $\kappa$ need not be additive) and satisfies $i_{0}^{*} \kappa=\operatorname{id}_{F}$, $i_{1}^{*} \kappa=0$. Then $\mathcal{C}^{\Delta}(F)$ is acyclic, i.e., $F \in I^{\Delta}$.

3.1.2. The story of 3.1.1 remains literally valid if we replace presheaves by presheaves with transfers. So we have subcategories $I_{\mathrm{tr}}^{\Delta}, I_{\mathrm{tr}}^{\Delta \perp} \subset D \mathcal{P} \mathcal{S} h_{\mathrm{tr}}$ of $\mathbb{A}^{1}$ homotopy contractible and $\mathbb{A}^{1}$-homotopy invariant presheaves with transfers, a DG endofunctor $F \mapsto \mathcal{C}^{\Delta}(F):=\varliminf_{\longrightarrow} \mathcal{H o m}\left(\sigma_{\leq n} R_{\mathrm{tr}}\left[\Delta^{\cdot}\right]^{\text {nor }}, F\right)$ of $C \mathcal{P} \mathcal{S} h_{\mathrm{tr}}$ which descends to the $I_{\mathrm{tr}}^{\Delta}$-localization endofunctor of $D \mathcal{P} \mathcal{S} h_{\mathrm{tr}}$, a non-degenerate t-structure on $I_{\mathrm{tr}}^{\Delta \perp} \stackrel{\sim}{\rightarrow} D \mathcal{P} \mathcal{S} h_{\mathrm{tr}} / I_{\mathrm{tr}}^{\Delta}$ whose core is the abelian category $\mathcal{P} \mathcal{S} h_{\mathrm{tr}}^{\Delta}$ of $\mathbb{A}^{1}$-homotopy invariant presheaves with transfers, etc.

Exercise. Show that $I_{\mathrm{tr}}^{\Delta \perp}$ is not equivalent to the derived category of $\mathcal{P} \mathcal{S} h_{\mathrm{tr}}^{\Delta} .{ }^{17}$

The $\mathbb{A}^{1}$-homotopy localization is compatible with functors (2.1.4). Namely, the functor $D \mathcal{P S} h \rightarrow D \mathcal{P S} h_{\mathrm{tr}}$ sends $I^{\Delta}$ to $I_{\mathrm{tr}}^{\Delta}$; its right adjoint (the forgetful functor) $D \mathcal{P S} h_{\mathrm{tr}} \rightarrow D \mathcal{P S h}$ commutes with $\mathcal{C}^{\Delta}$ (hence sends $I_{\mathrm{tr}}^{\Delta}$ to $I^{\Delta}, I_{\mathrm{tr}}^{\Delta \perp}$ to $I^{\Delta \perp}$ ).

As in 2.3, our triangulated categories carry natural DG structures. Namely, set $\mathcal{D}_{p \mathcal{M}}^{\text {eff }}:=\mathcal{P}_{\text {tr }} /{ }^{\kappa} \mathcal{I}_{\text {tr }}^{\Delta}$. Then, by $(1.8 .1)$, one has $\underline{\mathcal{D}_{p \mathcal{M}}^{\text {eff }}} \stackrel{\sim}{\rightarrow} \underline{\mathcal{P}_{\text {tr }}} / \underline{\mathcal{I}_{\text {tr }}^{\Delta}}$ and

$$
\stackrel{\mathcal{D}}{\text { eff }} \text { tri }_{\longrightarrow}^{\sim} D \mathcal{P} \mathcal{S} h_{\mathrm{tr}} / I_{\mathrm{tr}}^{\Delta} \stackrel{\sim}{\leftarrow} I_{\mathrm{tr}}^{\Delta \perp}
$$

Since $\mathcal{I}_{\text {tr }}^{\Delta}$ is a tensor ideal in $\mathcal{P}_{\text {tr }}$, our $\mathcal{D}_{p \mathcal{M}}^{\text {eff }}$ and $\underset{\mathcal{D}_{p \mathcal{M}}^{\text {eff }}}{\longrightarrow}$ are homotopy tensor DG categories. The endofunctor $\mathcal{C}^{\Delta}$ of $D \mathcal{P} \mathcal{S} h_{\text {tr }}$ is right t-exact. Hence the tensor product on $\stackrel{\mathcal{D}}{p \mathcal{M}}^{\text {eff }}$ tri is right t-exact.

Objects of $\underset{\mathcal{D}_{p \mathcal{M}}^{\mathrm{eff}}}{\longrightarrow}\left(\right.$ resp. $\left.\mathcal{D}_{p \mathcal{M}}^{\mathrm{eff}}\right)$ are called effective (geometric) premotives. The image $R_{\mathrm{tr}}^{\Delta}[X]$ of $R_{\mathrm{tr}}[X]$ in $\mathcal{D}_{p \mathcal{M}}^{\text {eff }}$ is called the premotive of $X$. We have the Tate premotive $R_{\mathrm{tr}}^{\Delta}(1)$, etc.

Remark. Let $\operatorname{Hot} \mathcal{C o r}^{\Delta}(\mathcal{S} m)$ be the full subcategory of $\mathcal{D}_{p \mathcal{M}}^{\text {eff tri }}$ formed by objects $R_{\mathrm{tr}}^{\Delta}[X], X \in \mathcal{S} m$, i.e., its morphisms $\mathcal{C}^{\Delta}{ }^{\Delta}(Y, X):=H^{0} \operatorname{Hom}\left(R_{\mathrm{tr}}^{\Delta}[Y], R_{\mathrm{tr}}^{\Delta}[X]\right)$ are $\mathbb{A}^{1}$-homotopy classes of correspondences. Then $\mathbb{A}^{1}$-homotopy invariant presheaves with transfers are the same as right $\operatorname{Hot} R_{\mathrm{tr}}^{\Delta}[\mathcal{S} m]$-modules, i.e., $R$-linear functors $F: \operatorname{Hot} R_{\text {tr }}^{\Delta}[\mathcal{S} m]^{\text {op }} \rightarrow R$-mod.

3.1.3. Let $X, Y$ be affine smooth varieties.

Question. Is it true that the complex $\operatorname{Hom}\left(R_{\mathrm{tr}}^{\Delta}[X], R_{\mathrm{tr}}^{\Delta}[Y]\right)=\mathcal{C}$ or $(X \times \Delta, Y)$ is acyclic in degrees $<-\operatorname{dim} Y$ ?

3.2. The Tate premotive. Here are some typical computations in $\mathcal{D}_{p \mathcal{M}}^{\text {eff }}$ :

\footnotetext{
${ }^{17}$ Hint: Since $R_{\mathrm{tr}}[X]$ is a projective object of $\mathcal{P S} h_{\mathrm{tr}}$ and the embedding $I_{\mathrm{tr}}^{\Delta \perp} \hookrightarrow D \mathcal{P} \mathcal{S} h_{\mathrm{tr}}$ is t-exact, $H^{0} \mathcal{C}^{\Delta}\left(R_{\mathrm{tr}}[X]\right)$ is a projective object in $\mathcal{P} \mathcal{S} h_{\mathrm{tr}}^{\Delta}$. Were $D \mathcal{P} \mathcal{S} h_{\mathrm{tr}}^{\Delta} \rightarrow I_{\mathrm{tr}}^{\Delta} \perp$ be an equivalence, this would imply that $\mathcal{C}^{\Delta}\left(R_{\mathrm{tr}}[X]\right) \stackrel{\sim}{\rightarrow} H^{0} \mathcal{C}^{\Delta}\left(R_{\mathrm{tr}}[X]\right)$.
} 
Proposition. (i) The $\mathbb{Z}$-presheaf with transfers $\mathcal{O}^{\times}$(see 2.1.3) is $\mathbb{A}^{1}$-homotopy invariant and there is a canonical homotopy equivalence in $\mathcal{D}_{p \mathcal{M}}^{\mathrm{eff}}$

$$
\epsilon_{R}: R_{\mathrm{tr}}^{\Delta}(1)[1] \stackrel{\sim}{\rightarrow} \mathcal{O}^{\times} \stackrel{L}{\otimes} R
$$

(ii) The transposition of factors action of the symmetric group $\Sigma_{n}$ on the premotive $R_{\mathrm{tr}}^{\Delta}(1)^{\otimes n}=R_{\mathrm{tr}}^{\Delta}(n)$ is homotopically trivial (i.e., is trivial in $\mathcal{D}_{p \mathcal{M}}^{\mathrm{eff}}$ ).

Proof. (i) The $\mathbb{A}^{1}$-homotopy invariance of $\mathcal{O}^{\times}$is clear (our varieties are reduced).

Let $\epsilon: \mathbb{Z}_{\operatorname{tr}}(1)[1] \rightarrow \mathcal{O}^{\times}$be a morphism of $\mathbb{Z}$-presheaves with transfers defined as the composition $\mathbb{Z}_{\mathrm{tr}}(1)[1] \hookrightarrow \mathbb{Z}_{\mathrm{tr}}\left[\mathbb{G}_{m}\right] \rightarrow \mathcal{O}^{\times}$, the latter arrow sends $\gamma \in \mathcal{C}$ or $\left(X, \mathbb{G}_{m}\right)$ to $\gamma^{*}(t) \in \mathcal{O}^{\times}(X)$ (see 2.1.3); here $t \in \mathcal{O}^{\times}\left(\mathbb{G}_{m}\right)$ is the standard parameter. Our $\epsilon$ is evidently surjective; we will check in a moment that its kernel $K$ is in $I^{\Delta}$. Since $\mathbb{Z}$-presheaves $K \subset \mathbb{Z}_{\mathrm{tr}}(1)[1] \subset \mathbb{Z}_{\mathrm{tr}}\left[\mathbb{G}_{m}\right]$ have no $\mathbb{Z}$-torsion, $\epsilon$ yields a quasiisomorphism $\mathcal{C}$ one $\left(K \otimes R \rightarrow R_{\mathrm{tr}}(1)[1]\right) \rightarrow \mathcal{O}^{\times} \stackrel{L}{\otimes} R$. Then $\epsilon_{R}$ is its composition with the embedding $R_{\mathrm{tr}}(1)[1] \rightarrow \mathcal{C}$ one $\left(K \otimes R \rightarrow R_{\mathrm{tr}}(1)[1]\right)$.

To show that $K \in I^{\Delta}$, we present $\epsilon$ as follows. For $X \in \mathcal{S} m$ a morphism $\mathbb{Z}_{\mathrm{tr}}[X] \rightarrow \mathbb{Z}_{\mathrm{tr}}(1)[1]$ is a divisor $D$ on $\mathbb{G}_{m X}$ finite over $X$ of relative degree 0 . Let $\Phi(X)$ be the multiplicative group of meromorphic functions $\phi$ on $\mathbb{P}_{X}^{1}$ such that $\phi$ is defined on a Zariski neighborhood of $\{0, \infty\} \times X, \phi(\infty, x) \equiv 1$, and $\phi(0, x)$ is invertible. One has an isomorphism $\Phi(X) \stackrel{\sim}{\rightarrow} \operatorname{Hom}\left(\mathbb{Z}_{\mathrm{tr}}[X], \mathbb{Z}_{\mathrm{tr}}(1)[1]\right), \phi \mapsto \operatorname{div} \phi$, and $\epsilon(\operatorname{div} \phi)(x)=\phi(0, x)$. Therefore $K$ equals the subgroup of $\phi \in \Phi$ with $\phi(0, x) \equiv$ 1. One has a natural map $\kappa: K(X) \rightarrow K\left(\mathbb{A}^{1} \times X\right), \kappa(\phi)(t, u, x):=u+(1-u) \phi(t, x)$ (here $u$ is the parameter on $\mathbb{A}^{1}$ ), which satisfies the conditions of the second exercise in 3.1.1, and we are done by that exercise.

(ii) It suffices to consider the case $n=2$. Let $\sigma$ be the transposition of factors of $\mathbb{G}_{m} \times \mathbb{G}_{m}$. We want to show that $\sigma+$ id kills the direct summand $\left(R_{\mathrm{tr}}^{\Delta}(1)[1]\right)^{\otimes 2} \subset$ $R_{\mathrm{tr}}^{\Delta}\left[\mathbb{G}_{m} \times \mathbb{G}_{m}\right]$ in the homotopy category, i.e., that $\sigma+\mathrm{id} \in \mathcal{C}$ or $\left(\mathbb{G}_{m} \times \mathbb{G}_{m}, \mathbb{G}_{m} \times \mathbb{G}_{m}\right)$ is $\mathbb{A}^{1}$-homotopic to the sum of correspondences that take values in $1 \times \mathbb{G}_{m}$ and in $\mathbb{G}_{m} \times 1$. Let $\alpha \in \operatorname{End}\left(\mathbb{G}_{m} \times \mathbb{G}_{m}\right)$ be the map $\left(x, x^{\prime}\right) \mapsto\left(x x^{\prime}, 1\right)$; we check that $\sigma+$ id is $\mathbb{A}^{1}$-homotopic to $\sigma \alpha+\alpha$. One has $\sigma+\mathrm{id}=p^{\vee} p$, where $p: \mathbb{G}_{m} \times \mathbb{G}_{m} \rightarrow \operatorname{Sym}^{2} \mathbb{G}_{m}$ is the projection, $p^{\vee}$ is the transposed correspondence. Since $\operatorname{Sym}^{2} \mathbb{G}_{m} \stackrel{\sim}{\rightarrow} \mathbb{G}_{m} \times \mathbb{A}^{1}$, $p\left(x, x^{\prime}\right) \mapsto\left(x x^{\prime}, x+x^{\prime}\right)$, our $p$ is $\mathbb{A}^{1}$-homotopic to $p \alpha$. Thus $\sigma+\mathrm{id}=p^{\vee} p$ is $\mathbb{A}^{1}$ homotopic to $p^{\vee} p \alpha=\sigma \alpha+\alpha$, q.e.d.

Question. By (ii) above, $R_{\mathrm{tr}}^{\Delta}(*):=\underset{n \geq 0}{\bigoplus} R_{\mathrm{tr}}^{\Delta}(n)$ is a commutative unital algebra in the tensor category $\underline{\mathcal{D}}_{p \mathcal{M}}^{\text {eff }}$ tri . Can one realize it naturally as an $E_{\infty}$-algebra in the homotopy tensor DG category $\stackrel{\mathcal{D}_{p \mathcal{M}}^{\mathrm{eff}}}{\longrightarrow}$ ?

Remark. The next generalization of the proposition can be proved by a simple modification of the argument. Let $C$ be a connected smooth projective curve over $k, A \subset C$ be a finite non-empty subscheme étale over $k$.

(i) One has a canonical homotopy equivalence $R_{\mathrm{tr}}^{\Delta}[C \backslash A] \stackrel{\sim}{\rightarrow} \operatorname{Pic}(C, A)$ in $\mathcal{D}_{p \mathcal{M}}^{\mathrm{eff}}$. Here $\operatorname{Pic}(C, A)$ is the generalized Jacobian (the group scheme of pairs $(\mathcal{L}, \iota), \mathcal{L}$ is a 
line bundle on $C, \iota$ is a trivialization of $\left.\mathcal{L}\right|_{A}$ ) considered as a presheaf with transfers (see 2.1.3); notice that this presheaf is $\mathbb{A}^{1}$-homotopy invariant.

(ii) (M. Nori, cf. [M] 5.8). Let $B \subset C \backslash A$ be another non-empty finite subscheme étale over $k$. Set $M(C ; A, B):=\mathcal{C}$ one $\left(\mathbb{Z}_{\mathrm{tr}}^{\Delta}[B] \rightarrow \mathbb{Z}_{\mathrm{tr}}^{\Delta}[C \backslash A]\right) \in \mathcal{D}_{\mathcal{M}}^{\text {eff }}$. Consider the action of the symmetric group $\Sigma_{n}$ on $M(C ; A, B)^{\otimes n}$; let $\tau_{n} \in \operatorname{End}\left(M(C ; A, B)^{\otimes n}\right)$ be the $\Sigma_{n}$-averaging (the sum of operators $s, s \in \Sigma_{n}$ ). Then $\tau_{n}$ is naturally homotopic to 0 if $n>2 g-2+|A|+|B|$; here $g$ is the genus of $C$.

3.3. Theorem. The Tate premotive is homotopically quasi-invertible: on $\mathcal{D}_{p \mathcal{M}}^{\mathrm{eff}}$ (or $\mathcal{D}_{p \mathcal{M}}^{\mathrm{eff}}$ ) the Tate twist endofunctor $M \mapsto M(1)$ is homotopically fully faithful.

Remark. For $F \in D \mathcal{P S} h_{\text {tr }}$ let $\nu_{F}: F \rightarrow\left(\mathcal{C}^{\Delta}(F(1))\right)_{-1}[-1]$ be obtained by adjunction (see 2.2) from the canonical morphism $F(1) \rightarrow \mathcal{C}^{\Delta}(F(1))$. The theorem means that $\nu_{F}$ is a quasi-isomorphism if $F$ is $\mathbb{A}^{1}$-homotopically invariant.

Proof of the theorem. We want to show that the Tate twist map $\operatorname{Hom}\left(M^{\prime}, M\right) \rightarrow$ $\operatorname{Hom}\left(M^{\prime}(1), M(1)\right)$ is a quasi-isomorphism for every premotives $M, M^{\prime}$. It suffices to consider the case when $M, M^{\prime}$ are the premotives of some $Z, Z^{\prime} \in \mathcal{S} m$. We can assume that $R=\mathbb{Z}$. Notice that for $Z=\operatorname{Spec} k$ the statement follows from 3.2(i).

As in 3.1, we represent $\operatorname{Hom}\left(\mathbb{Z}_{\mathrm{tr}}^{\Delta}\left[Z^{\prime}\right], \mathbb{Z}_{\mathrm{tr}}^{\Delta}[Z]\right)$ by the complex $C=C\left(Z^{\prime}, Z\right)$ with components $C^{a}=\mathcal{C}$ or $\left(Z^{\prime} \times \Delta^{a}, Z\right)$. Set $C^{b}=C^{b}\left(Z^{\prime}, Z\right):=C\left(Z^{\prime} \times \mathbb{G}_{m}, Z \times \mathbb{G}_{m}\right)$, $E:=\operatorname{Cor}\left(\mathbb{G}_{m}, \mathbb{G}_{m}\right)=\operatorname{End}\left(\mathbb{Z}_{\mathrm{tr}}\left[\mathbb{G}_{m}\right]\right) ;$ our $C^{b}$ is an $E$-bimodule. Let $\tau \in E$ be the correspondence $x \mapsto(x)-(1)$; this is a projector onto $\mathbb{Z}_{\mathrm{tr}}(1)[1] \subset \mathbb{Z}_{\mathrm{tr}}\left[\mathbb{G}_{m}\right]$. Let $C_{\tau}^{b}$ be the direct summand $\tau C^{b} \tau$ of $C^{b}, E_{\tau}:=\tau E \tau=\operatorname{End}\left(\mathbb{Z}_{\operatorname{tr}}(1)\right)$, so $C_{\tau}^{b}$ is a $E_{\tau}$-bimodule. The Tate twist map is represented as $\rho: C \rightarrow C_{\tau}^{b}, \gamma \mapsto \gamma \otimes \tau$. Let us show that $\rho$ is a quasi-isomorphism.

3.3.1. $\rho$ is injective on cohomology: For $n \geq 1$ let $\iota_{n} \in E$ be the correspondence $x \mapsto x^{1 / n}$ (so $\operatorname{deg}\left(\iota_{n}\right)=n$ ); one has $\iota_{m n}=\iota_{m} \iota_{n}$. Notice that the image of $\tau \iota_{n} \tau=$ $\iota_{n} \tau \in E_{\tau}$ in $H^{0} \operatorname{End}\left(\mathbb{Z}_{\mathrm{tr}}^{\Delta}(1)\right)=\mathbb{Z}$ equals 1 , so the left multiplication by $\iota_{n}$ preserves $C_{\tau}^{b}$ and is homotopic to the identity on it.

We will define a subcomplex $C^{\natural} \subset C^{b}$ preserved by the $\iota_{n}$, and a morphism of complexes $\kappa: C^{\natural} \rightarrow C$ with the next properties: (a) For any $\chi \in C^{b}$ one has $\iota_{n} \chi \in C^{\natural}$ for $n \gg 1$; (b) For any $\gamma \in C$ and $n>1$ one has $\iota_{n} \rho(\gamma) \in C^{\natural}$ and $\kappa\left(\iota_{n} \rho(\gamma)\right)=\gamma$. This yields the injectivity of $H^{\cdot} \rho$.

For a smooth $Y$ (we will need $Y=Z^{\prime} \times \Delta^{*}$ ) consider $D \in \mathcal{C}$ or $\left(Y \times \mathbb{G}_{m}, \mathbb{G}_{m}\right)$ given by an effective divisor. Let $t, t^{\prime} \in \mathcal{O}^{\times}\left(\mathbb{G}_{m} \times\left(Y \times \mathbb{G}_{m}\right)\right)$ be the projections to the two $\mathbb{G}_{m}$-factors. Locally on $Y$ we can write $D=(f=0)$ where $f=f\left(t, t^{\prime}\right) \in \mathcal{O}_{Y}\left(t, t^{\prime}\right)$ is a Laurent polynomial. The condition on $D$ says that its closure in $\mathbb{P}^{1} \times\left(Y \times \mathbb{G}_{m}\right)$ does not meet the divisor $t=\{0, \infty\}$. This means that, after multiplication by an invertible function on $Y$ and powers of $t, t^{\prime}$, our $f$ can be written as $1+p_{0}\left(t, t^{\prime}\right)$ and as $1+p_{\infty}\left(t^{-1}, t^{\prime-1}\right)$ where $p_{0}, p_{\infty} \in t \mathcal{O}_{Y}\left[t, t^{\prime}, t^{\prime-1}\right]$. We say that $D$ is nice if $p_{0}, p_{\infty}$ are sums of monomials of degree $>0$. This condition assures that $D \cap\left(t=t^{\prime}\right) \subset \mathbb{G}_{m} \times Y$ (here $\mathbb{G}_{m}$ is the diagonal $t=t^{\prime}$ ) is closed in $\mathbb{P}^{1} \times Y$, hence it is finite over $Y$.

Now our $C^{\natural} \subset C^{b}$ is generated by those effective correspondences $\chi \in C^{b}$ that $\pi \chi \in \operatorname{Cor}\left(Z^{\prime} \times \Delta^{a} \times \mathbb{G}_{m}, \mathbb{G}_{m}\right)$ is nice; here $\pi$ is the projection $Z \times \mathbb{G}_{m} \rightarrow \mathbb{G}_{m}$. By the above, any $\chi \in C^{\natural}$ meets the divisor $t=t^{\prime}$ on $\left(Z \times \mathbb{G}_{m}\right) \times\left(Z^{\prime} \times \mathbb{G}_{m}\right)$ properly, 
and the cycle-theoretic intersection $\chi_{\text {diag }}:=\chi \cap\left(t=t^{\prime}\right)$ is finite over $Z^{\prime}$. We define $\kappa(\chi) \in C$ as the image of $-\chi_{\text {diag }}$ by the projection to $Z \times Z^{\prime} \times \Delta^{a}$. Properties (a), (b) are evident.

3.3.2. $\rho$ is surjective on cohomology: Let $\chi \in C_{\tau}^{b}$ be any cycle. Let us show that for sufficiently large $n$ our $\chi$ is homotopic to $\rho \kappa\left(\iota_{n} \chi\right)$.

Consider another copy $\mathbb{G}_{m}^{c}$ of $\mathbb{G}_{m}$; let $\tau^{c}$ be the projector $\tau$ on it, etc. Let us perform the above constructions replacing $Z^{(\prime)}$ by $Z^{(\prime)} \times \mathbb{G}_{m}$ and $\mathbb{G}_{m}$ by $\mathbb{G}_{m}^{c}$. We get a subcomplex $C^{\natural}\left(Z^{\prime} \times \mathbb{G}_{m}, Z \times \mathbb{G}_{m}\right) \subset C^{b}\left(Z^{\prime} \times \mathbb{G}_{m}, Z \times \mathbb{G}_{m}\right)$, a morphism $\kappa^{c}: C^{\natural}\left(Z^{\prime} \times \mathbb{G}_{m}, Z \times \mathbb{G}_{m}\right) \rightarrow C\left(Z^{\prime} \times \mathbb{G}_{m}, Z \times \mathbb{G}_{m}\right)$, etc.

Let $\sigma$ be the transposition symmetry of $\mathbb{G}_{m} \times \mathbb{G}_{m}^{c}$. By 3.2(ii), one has $\chi \otimes \tau^{c}-$ $\sigma\left(\chi \otimes \tau^{c}\right) \sigma=d \phi$ for some $\phi \in C\left(Z^{\prime} \times \mathbb{G}_{m} \times \mathbb{G}_{m}^{c}, Z \times \mathbb{G}_{m} \times \mathbb{G}_{m}^{c}\right)$ which is invariant with respect to the left and right multiplications by $\tau, \tau^{c}$. Take any $n \geq 2$ such that $\iota_{n}^{c} \phi \in C^{\natural}\left(Z^{\prime} \times \mathbb{G}_{m}, Z \times \mathbb{G}_{m}\right)$; then $\iota_{n}^{c}\left(\chi \otimes \tau^{c}\right), \iota_{n}^{c} \sigma\left(\chi \otimes \tau^{c}\right) \sigma$ lie in this subcomplex as well. Since $\chi=\kappa^{c}\left(\iota_{n}^{c}\left(\chi \otimes \tau^{c}\right)\right)$ and $\rho \kappa\left(\iota_{n} \chi\right):=\kappa\left(\iota_{n} \chi\right) \otimes \tau=\kappa^{c}\left(\iota_{n}^{c} \sigma\left(\chi \otimes \tau^{c}\right) \sigma\right)$, one has $\chi-\rho \kappa\left(\iota_{n} \chi\right)=d \kappa^{c}\left(\iota_{n}^{c} \phi\right)$, q.e.d.

3.4. Specialization and Tate untwisting. 3.4.1. Let $X$ be a smooth variety, $U \subset X$ its dense open subset. We call such a datum simply a pair, and denote it by $(X, U)$. If $\left(X^{\prime}, U^{\prime}\right)$ is another pair, then a correspondence $\theta:\left(X^{\prime}, U^{\prime}\right) \rightarrow(X, U)$ is a correspondence $\theta \in \mathcal{C} \operatorname{or}\left(X^{\prime}, X\right)$ such that the restriction $\theta^{\circ}$ of $\theta$ to $U^{\prime}$ lies in $\mathcal{C} \operatorname{or}\left(U^{\prime}, U\right) \subset \mathcal{C} \operatorname{Cor}\left(U^{\prime}, X\right)$. We have an $R$-category $\operatorname{Cor}(\mathcal{P} a)$ whose objects are pairs and morphisms are correspondences $\operatorname{Cor}\left(\left(X^{\prime}, U^{\prime}\right),(X, U)\right)$. One has two evident faithful functors $\operatorname{Cor}(\mathcal{P} a) \rightarrow \mathcal{C} o r(\mathcal{S} m),(X, U) \mapsto X, U$, and a functor $\operatorname{Cor}(\mathcal{P} a) \rightarrow$ $\mathcal{P}_{\mathrm{tr}},(X, U) \mapsto R_{\mathrm{tr}}[U / X]:=\mathcal{C}$ one $\left(R_{\mathrm{tr}}[U] \rightarrow R_{\mathrm{tr}}[X]\right)[-1]$. For $\theta$ as above we denote the morphism $R_{\mathrm{tr}}\left[U^{\prime} / X^{\prime}\right] \rightarrow R_{\mathrm{tr}}[U / X]$ by $\theta^{\text {rel }}$.

A pair $(X, U)$ is said to be smooth if $Z:=X \backslash U$ is a smooth divisor. Let $\operatorname{Cor}\left(\mathcal{P} a^{\mathrm{sm}}\right)$ be the subcategory of smooth pairs. If $\theta$ as above is a correspondence between smooth pairs, then the divisor $Z \times X^{\prime}$ in $X \times X^{\prime}$ meets $\theta$ properly. Set

$$
S p(\theta):=\theta \cap\left(Z \times X^{\prime}\right)
$$

where the intersection is computed in the cycle-theoretic sense. This is a cycle supported at $Z \times Z^{\prime} \subset X \times X^{\prime}$ and finite over $Z^{\prime}$. Therefore $\operatorname{Sp}(\theta) \in \mathcal{C} \operatorname{or}\left(Z^{\prime}, Z\right)$.

We leave it to the reader to check that the specialization is compatible with the composition, i.e., $S p\left(\theta \theta^{\prime}\right)=S p(\theta) S p\left(\theta^{\prime}\right)$. Thus we have a functor $S p: \mathcal{C}$ or $\left(\mathcal{P} a^{\mathrm{sm}}\right) \rightarrow$ $\operatorname{Cor}(\mathcal{S} m),(X, U) \mapsto Z, \theta \mapsto S p(\theta)$.

Remark. If $\theta \in \mathcal{C}$ or $\left(X^{\prime}, U\right) \subset \mathcal{C}$ or $\left(\left(X^{\prime}, U^{\prime}\right),(X, U)\right)$, then $\theta^{r e l}$ is homotopic to 0 and $\operatorname{Sp}(\theta)=0$.

3.4.2. We call $\mathbb{T}:=\left(\mathbb{A}^{1}, \mathbb{G}_{m}\right)$ the Tate pair. So for every smooth $Z$ we have a smooth pair $Z \times \mathbb{T}:=\left(Z \times \mathbb{A}^{1} / Z \times \mathbb{G}_{m}\right)$. One has a canonical homotopy equivalence $R_{\mathrm{tr}}^{\Delta}\left[Z \times \mathbb{G}_{m} / Z \times \mathbb{A}^{1}\right] \stackrel{\sim}{\rightarrow} R_{\mathrm{tr}}^{\Delta}[Z](1)[1]$.

Take any $\theta \in \operatorname{Cor}\left(Z^{\prime} \times \mathbb{T}, Z \times \mathbb{T}\right)$. By the above homotopy equivalence, we can consider $\theta^{r e l}$ as a homotopy morphism $R_{\mathrm{tr}}^{\Delta}\left[Z^{\prime}\right](1) \rightarrow R_{\mathrm{tr}}^{\Delta}[Z](1)$. We also have $S p(\theta): R_{\mathrm{tr}}\left[Z^{\prime}\right] \rightarrow R_{\mathrm{tr}}[Z]$.

Proposition. $\theta^{\text {rel }}$ is $\mathbb{A}^{1}$-homotopic to $S p(\theta)(1)$. 
Proof. We use the notation from 3.3. Notice that $\iota_{n}, \tau \in E$ from loc. cit. come from correspondences in $\operatorname{Cor}(\mathbb{T}, \mathbb{T})$ which we denote by $\tilde{\iota}_{n}, \tilde{\tau}$. By the remark above, replacing $\theta$ by $\tilde{\tau} \theta \tilde{\tau}$ does not change $\theta^{r e l}$ and $S p(\theta)$, so we can assume that $\theta=\tilde{\tau} \theta \tilde{\tau}$. Then $\theta^{r e l}$ is homotopic to $\theta^{o} \in C_{\tau}^{b}$. By 3.3.2 applied to $\chi=\theta^{\circ}$, it remains to show that $S p(\theta)$ is homotopic to $\kappa\left(\iota_{n} \theta^{\circ}\right)$ for sufficiently large $n$.

Set $\tilde{C}^{b}:=C\left(Z^{\prime} \times \mathbb{A}^{1}, Z \times \mathbb{A}^{1}\right)$. We define a subcomplex $\tilde{C}^{\natural} \subset \tilde{C}^{b}$ and the morphism $\tilde{\kappa}: \tilde{C}^{\natural} \rightarrow C\left(Z^{\prime}, Z\right)$ in the same way as we have defined $C^{\natural}, \kappa$ in 3.3.1 replacing $\mathbb{G}_{m}$ by $\mathbb{A}^{1}$ (there is a single difference: to define the nice correspondences in $\operatorname{Cor}\left(Y \times \mathbb{A}^{1}, \mathbb{A}^{1}\right)$, we look at $p_{\infty}$ only). As in loc. cit., for any $\theta^{\prime} \in \tilde{C}^{b}$ one has $\tilde{\iota}_{n} \theta^{\prime} \in \tilde{C}^{\natural}$ for sufficiently large $n$. For any $\gamma \in C$ one has $\tilde{\iota}_{n}(\gamma \otimes \tilde{\tau}) \in \tilde{C}^{\natural}$, but, at variance with $\kappa$, one has $\tilde{\kappa} \tilde{\iota}_{n}(\gamma \otimes \tilde{\tau})=0$ (for $\tilde{\kappa}$ includes the intersection with the diagonal $t=t^{\prime}$ at $t=0$ ).

Since $\tilde{\tau}$ is $\mathbb{A}^{1}$-homotopic to zero in $\operatorname{Cor}\left(\mathbb{A}^{1}, \mathbb{A}^{1}\right)$ (for its degree equals 0 ), so is $\theta=\tilde{\tau} \theta$, i.e., $\theta=d \psi$ in the complex $\tilde{C}^{b}$. Thus $\tilde{\kappa}\left(\tilde{\iota}_{n} \theta\right)=d\left(\tilde{\kappa}\left(\tilde{\iota}_{n} \psi\right)\right)$ for large $n$. Now $\tilde{\kappa}\left(\tilde{\iota}_{n} \theta\right)=\kappa\left(\iota_{n} \theta^{o}\right)-S p^{\prime}\left(\tilde{\iota}_{n} \theta\right)$ where $S p^{\prime}\left(\tilde{\iota}_{n} \theta\right) \in \mathcal{C} \operatorname{or}\left(Z^{\prime}, Z\right)$ is the intersection of the cycle $\tilde{\iota}_{n} \theta$ with the diagonal $t=t^{\prime}$ at $t=0$. The latter equals the intersection of $\theta$ with the divisor $t^{n}=t^{\prime}$ at $t=0$, which is $S p(\theta)$ for large $n$, and we are done.

\section{$\S 4$. The motivic localization}

This section deals with the subject of lectures 12, 13, 24 of [MVW] and 3.1, 3.2 of [Vo2]. The principal result is the theorem in 4.4 (cf. [Vo2] 3.2.6, 3.1.12, [MVW] 13.8, 13.9, [Deg2] 5.1). We deduce it from the theory of residues (the Cousin resolution) for $\mathbb{A}^{1}$-homotopy invariant presheaves with transfers. The latter is formulated in 4.6, the proofs are in $§ 5$. The original argument of Voevodsky is rather different (the Cousin resolution emerges in the aftermath on the last page of $[\mathrm{MVW}]$ ), though both proofs are based on the same key input (see 5.1, 5.3).

4.1. The Zariski localization: a complaint. The Zariski localization of plain presheaves is a particular case of the format from 1.11 for categories of schemes such as $\mathcal{S} m$ equipped with the Zariski topology. The local objects for the Zariski topology are local schemes (which are localizations of the schemes from our category). A complex of presheaves is Zariski locally acyclic (see 1.11) if and only if it is stalkwise acyclic. The subcategory $I^{\mathrm{Zar}}$ in $D \mathcal{P} \mathcal{S} h$ of Zariski locally acyclic complexes is generated by all Mayer-Vietoris complexes $R[U \cap V] \rightarrow R[U] \oplus R[V] \rightarrow R[X]$ (here $U, V \subset X$ are opens such that $U \cup V=X$ ), see [BG] (or modify the proof of the proposition in 4.2.1 for the Zariski site).

Passing to presheaves with transfers, we see that the category $I_{\mathrm{tr}}^{\mathrm{Zar} \perp} \subset D \mathcal{P} \mathcal{S} h_{\mathrm{tr}}$ from 2.3 consists of all $F$ 's that are Zariski local as complexes of mere presheaves. But the category $I_{\mathrm{tr}}^{\text {Zar }}$ itself contains not only all complexes which are Zariski stalkwise acyclic, but many other objects as well: e.g. the Mayer-Vietoris complex $R_{\mathrm{tr}}[U \cap V] \rightarrow R_{\mathrm{tr}}[U] \oplus R_{\mathrm{tr}}[V] \rightarrow R_{\mathrm{tr}}[X]$ is not Zariski stalkwise acyclic if $X$ is irreducible and $U, V \neq X$. So the forgetful functor $o: D \mathcal{P S} h_{\mathrm{tr}} \rightarrow D \mathcal{P S} h$ does not send $I_{\mathrm{tr}}^{\mathrm{Zar}}$ to $I^{\mathrm{Zar}}$, hence it does not commute with the Zariski localization, which makes the Zariski localization of arbitrary presheaves with transfers unwieldy.

The cause of the nuisance is that the image of a local scheme by a correspondence is merely a semi-local scheme which need not be the disjoint union of local schemes. 
Thus a correspondence may not be continuous for the Zariski topology: the Zariski coverings do not define a Grothendieck topology on $\mathcal{C}$ or $(\mathcal{S} m)$.

The problem disappears if one replaces the Zariski topology by the Nisnevich one (see the lemma in 4.3). We will see that for perfect $k$ this does not change the motivic localization.

4.2. A Nisnevich localization reminder. For details, see [Ni] or [TT] App. E. To fix the setting, we play with the category $\mathcal{S} m$; it can be replaced by the category of all $k$-varieties (or all Noetherian schemes of finite Krull dimension).

Remark. The constructions below work with obvious modification (replacing étale maps by local isomorphisms) for the Zariski topology.

4.2.1. The Nisnevich topology lies in between the Zariski and étale topologies. A Nisnevich covering of $X$ is an étale covering $U \rightarrow X$ such that for every field $K$ the map $U(K) \rightarrow X(K)$ is surjective, or, equivalently, $U / X$ admits a constructible section (see 4.2.2). The local objects for the Nisnevich topology are Henselian local schemes.

$\mathcal{S} m$ equipped with the Nisnevich topology satisfies the conditions from 1.11, so the general format from loc. cit. readily applies. We have the abelian category of Nisnevich sheaves $\mathcal{S} h^{\mathrm{Nis}}$, the exact sheafification functor $\mathcal{P} \mathcal{S} h \rightarrow \mathcal{S} h^{\mathrm{Nis}}, F \mapsto$ $F_{\text {Nis }}$, the right-admissible subcategory $I^{\text {Nis }} \subset D \mathcal{P S} h$ of Nisnevich locally acyclic complexes, ${ }^{18}$ the category $I^{\mathrm{Nis} \perp}$ of Nisnevich local complexes, and the equivalences of the triangulated categories

$$
D \mathcal{P S h} / I^{\mathrm{Nis}} \stackrel{\sim}{\rightarrow} D \mathcal{S} h^{\mathrm{Nis}} \stackrel{\sim}{\leftarrow} I^{\mathrm{Nis} \perp} .
$$

Exercise. Suppose $\ell$ is prime to char $k$. Show that the presheaf $X \mapsto H_{e ́ t}^{1}\left(X, \mu_{\ell}\right)$ is a Nisnevich sheaf; if $k$ is separably closed, then the stalk $H_{e ̂ t}^{1}\left(X_{x}^{h}, \mu_{\ell}\right)$ vanishes if and only if $x$ is a closed point of $X .{ }^{19}$

Suppose we have an étale covering $\{U, V\}$ of $X \in \mathcal{S} m$ such that $V$ is an open subset of $X$, and for $Z:=X \backslash V$ one has $U_{Z}:=U \times_{X} Z \stackrel{\sim}{\rightarrow} Z$. We call such $\{U, V\}$ a Nisnevich Mayer-Vietoris covering, and the corresponding complex of presheaves $R\left[U_{V}\right] \rightarrow R[U] \oplus R[V] \rightarrow R[X]$ (the differentials are the difference and the sum of evident maps) a Nisnevich Mayer-Vietoris complex. It lies in $I^{\mathrm{Nis}}$.

Proposition. $I^{\mathrm{Nis}}$ is generated by the Nisnevich Mayer-Vietoris complexes. ${ }^{20}$

Proof. By (i) of the proposition in 1.4.2 (for $D=I^{\mathrm{Nis}}, S$ the set of all Nisnevich Mayer-Vietoris complexes), the assertion amounts to the next statement: Suppose $F \in I^{\mathrm{Nis}}$ is such that for each Nisnevich Mayer-Vietoris covering as above the total

\footnotetext{
${ }^{18}$ A complex $F$ of presheaves is Nisnevich locally acyclic iff it is Nisnevich stalkwise acyclic, i.e., for any $X \in \mathcal{S}, x \in X$ the complex $F\left(X_{x}^{h}\right)$ is acyclic; here $X_{x}^{h}$ is the Henselization of $X$ at $x$ considered as a proobject of $\mathcal{S} m$.

${ }^{19}$ This sheaf was denoted by $\mathcal{O}^{\times} / \ell$ in $[\mathrm{MVW}] 12.9$. The exercise shows that the formula from [MVW] 12.10 should be corrected: the product must take into account all points $x \in X$, not only the closed ones as in loc. cit.

${ }^{20}$ See [Vo4] for a more general story.
} 
complex of $F(X) \rightarrow F(U) \oplus F(V) \rightarrow F\left(U_{V}\right)$ is acyclic; then $F$ is acyclic. To show this, we check by induction by $n$ the next claim: For every $X$ and $h \in H^{a} F(X)$ there is an open $V \subset X$ with $X \backslash V$ of codimension $\geq n$ such that $\left.h\right|_{V} \in H^{a} F(V)$ vanishes.

By the induction assumption, there is an open $V^{\prime} \subset X$ with $Z:=X \backslash V^{\prime}$ of codimension $\geq n-1$ such that $\left.h\right|_{V^{\prime}}=0$. Let $z_{i}$ be the generic points of $Z$ of codimension $n-1$. Since $F \in I^{\text {Nis }}$, there is a Nisnevich covering of $X$ on which $h$ vanishes. Thus there is an open $V^{\prime \prime} \subset X$ containing $V^{\prime}$ and $z_{i}$ 's, and an étale $U^{\prime} / V^{\prime \prime}$ such that $U_{V^{\prime \prime} \cap Z}^{\prime} \stackrel{\sim}{\rightarrow} V^{\prime \prime} \cap Z$ and $\left.h\right|_{U^{\prime}}=0$. Then $\left\{U^{\prime}, V^{\prime}\right\}$ is a Nisnevich Mayer-Vietoris covering of $V^{\prime \prime}$, so the exact sequence $H^{a-1} F\left(U_{V^{\prime}}^{\prime}\right) \rightarrow$ $H^{a} F\left(V^{\prime \prime}\right) \rightarrow H^{a} F\left(U^{\prime}\right) \oplus H^{a} F\left(V^{\prime}\right)$ shows that $\left.h\right|_{V^{\prime \prime}}$ comes from $g \in H^{a-1} F\left(U_{V^{\prime}}^{\prime}\right)$. By the induction assumption, there is a closed $Y^{\prime} \subset U_{V^{\prime}}^{\prime}$ such that the image of $g$ in $H^{a-1} F\left(U_{V^{\prime}}^{\prime} \backslash Y^{\prime}\right)$ vanishes. Let $Y$ be the closure of the image of $Y^{\prime}$ in $X$. Then $V:=V^{\prime \prime} \backslash Z \cap Y$ is the promised open subset of $X$. Indeed, $X \backslash V$ has codimension $\geq n$ since $z_{i} \notin Z \cap Y$. Set $U:=U_{V^{\prime \prime} \backslash Y}^{\prime}$; then $\left\{U, V^{\prime}\right\}$ is a Nisnevich Mayer-Vietoris covering of $V$, so $\left.h\right|_{V}$ comes from $\left.g\right|_{U_{V^{\prime}}} \in H^{a-1} F\left(U_{V^{\prime}}\right)$. Since $U_{V^{\prime}} \subset U_{V^{\prime}}^{\prime} \backslash Y^{\prime}$, one has $\left.g\right|_{U_{V^{\prime}}}=0$, hence $\left.h\right|_{V}=0$, q.e.d.

In particular, we see that $I^{\mathrm{Nis}}$ is generated by a set of compact objects, so the Nisnevich localization fits into the picture of the proposition from 1.4.2.

Exercises. (i) A presheaf $F$ is a Nisnevich sheaf iff for every Nisnevich MayerVietoris covering the sequence $0 \rightarrow F(X) \rightarrow F(U) \oplus F(V) \rightarrow F\left(U_{V}\right)$ is exact.

(ii) For any Nisnevich sheaf $F$ and $h \in H_{\mathrm{Nis}}^{n}(X, F)$ there is a closed $Y \subset X$ of codimension $\geq n$ such that the image of $h$ in $H_{\mathrm{Nis}}^{n}(X \backslash Y, F)$ vanishes.

4.2.2. As was mentioned in 1.11, the Nisnevich localization functor $D \mathcal{P S} h \rightarrow$ $D \mathcal{S} h^{\mathrm{Nis}} \stackrel{\sim}{\rightarrow} I^{\mathrm{Nis} \perp} \subset D \mathcal{P S} h$ can be lifted to the level of complexes by means of the Godement resolution. A characteristic feature of the Nisnevich (as well as the Zarisky) topology is that the Godement resolution admits here an economic adelestyle version $\mathcal{C}^{\text {Nis }}{ }^{21}$ In the rest of the section we construct $\mathcal{C}^{\text {Nis }}$. We will not use it any heavily, so the reader who is not inclined to follow the details of the construction may pass directly to 4.3 .

For $X \in \mathcal{S} m$ a partition $\left\{Z_{\alpha}\right\}$ of $X$ always means a (finite) partition by locally closed reduced subschemes. For an $X$-scheme $Y / X$ a constructible section is a set-theoretic section $s: X \rightarrow Y$ such that for some partition $\left\{Z_{\alpha}\right\}$ of $X$ each $\left.s\right|_{Z_{\alpha}}: Z_{\alpha} \rightarrow Y$ is a morphism of schemes. ${ }^{22}$

For $X \in \mathcal{S} m$ consider the category $\Xi(X)$ of pairs $\xi=\left(U^{\xi} / X, s^{\xi}\right)$, where $U^{\xi} / X$ is an étale $X$-scheme and $s^{\xi}$ is a constructible section of $U^{\xi} / X$; a morphism $\xi \rightarrow \xi^{\prime}$ is a morphism of $X$-schemes $U^{\xi} \rightarrow U^{\xi^{\prime}}$ which sends $s^{\xi}$ to $s^{\xi^{\prime}}$. Let $\Xi_{o}(X)$ be the subcategory of those $\xi_{o}$ that each connected component of $U^{\xi_{o}}$ intersects $s^{\xi_{o}}(X)$. Our $\Xi(X)$ admits finite products, for any $\xi$, $\xi_{o}$ as above there is at most one morphism $\xi_{o} \rightarrow \xi$, and for each $\xi$ one can find some $\xi_{o} \rightarrow \xi$. Thus $\Xi(X)^{\text {op }}$ is directed, $\Xi_{o}(X)$ is its cofinal subset. Set $X_{0}^{\mathrm{Nis}}:=\varliminf_{\Xi(X)} U^{\xi}=\varliminf_{\Xi_{o}(X)} U^{\xi}$; this is

\footnotetext{
${ }^{21}$ Here "economic" means, in particular, that for a sheaf $F$ on a variety $X$ of dimension $n$ the resolution $\mathcal{C}^{\mathrm{Nis}}(F)$ has length $\leq n$.

${ }^{22}$ I.e., the closure of $s\left(Z_{\alpha}\right)$ in $Y_{Z_{\alpha}}$, considered as a closed reduced subscheme in $Y_{Z_{\alpha}}$, projects isomorphically to $Z_{\alpha}$.
} 
a proobject of $\mathcal{S} m / X$.

It fits into a simplicial proobject $X^{\mathrm{Nis}} / X$ defined as follows. For $n \geq 0$ all collections $\left(\xi_{0}, \ldots, \xi_{n}\right)$ where $\xi_{i}=\left(U^{\xi_{i}} / U^{\xi_{i-1}}, s^{\xi_{i}}\right) \in \Xi\left(U^{\xi_{i-1}}\right)$ (we set $U^{\xi_{-1}}:=X$ ) form a directed category $\Xi^{n}(X)$; $\xi$ 's with every $\xi_{i}$ in $\Xi_{o}$ comprise a directed set $\Xi_{o}^{n}(X)$ cofinal in $\Xi^{n}(X)$. Our $\Xi^{\cdot}(X)$ is a cosimplicial system of categories: for a monotone map $\phi:[0, n] \rightarrow[0, m]$ the corresponding functor $\Xi^{n}(X) \rightarrow \Xi^{m}(X)$

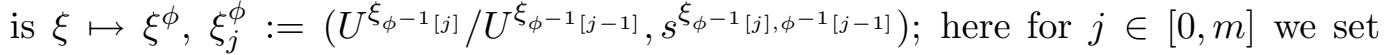
$\phi^{-1}[j]:=\max \{i: \phi(i) \leq j\} \in[0, n]$ and $s^{\xi_{a, b}}$ is the composition $s^{\xi_{a}} \ldots s^{\xi_{b+1}}$ if $a>b$ and the identity map if $a=b$. Now $X_{n}^{\mathrm{Nis}}:=\varliminf_{\Xi^{n}(X)} U^{\xi_{n}}$; the simplicial structure maps on $X_{.}^{\mathrm{Nis}} / X$ are $\phi^{\mathrm{Nis}}: X_{m}^{\mathrm{Nis}} \rightarrow X_{n}^{\mathrm{Nis}}$ defined as the $\Xi^{n}(X)$-projective limit of the maps $X_{m}^{\mathrm{Nis}} \rightarrow U^{\xi_{m}^{\phi}}=U^{\xi_{n}}$.

Any morphism of varieties $f: Y \rightarrow X$ lifts naturally to a morphism of simplicial proobjects $f^{\mathrm{Nis}}: Y^{\mathrm{Nis}} \rightarrow X^{\mathrm{Nis}}$ (for one has an evident pull-back functor $\left.f^{*}: \Xi^{\cdot}(X) \rightarrow \Xi^{\cdot}(Y)\right)$. Thus any presheaf $F$ yields a cosimplicial presheaf $F_{\text {Nis }}^{\cdot}$, $F_{\text {Nis }}^{\cdot}(X):=F\left(X^{\text {Nis }}\right)$ (i.e., $F_{\mathrm{Nis}}^{n}(X)=\varliminf_{\Xi^{n}(X)} F\left(U^{\xi_{n}}\right)$ ), augmented by $F$. Let $\mathcal{C}^{\mathrm{Nis}}(F)$ be its normalized complex and $\epsilon: F \rightarrow \mathcal{C}^{\mathrm{Nis}}(F)$ be the augmentation.

Proposition. (i) The functor $F \mapsto \mathcal{C}^{\mathrm{Nis}}(F)$ is exact. (ii) Each presheaf $\mathcal{C}^{\mathrm{Nis}}(F)^{n}$ is Nisnevich local (see 1.11), hence is a Nisnevich sheaf. (iii) The augmentation $\epsilon$ makes $\mathcal{C}^{\mathrm{Nis}}(F)$ a resolution of the Nisnevich sheafification of $F$. (iv) Every section of $\mathcal{C}^{\mathrm{Nis}}(F)^{n}$ is supported in codimension $n$, so $\mathcal{C}^{\mathrm{Nis}}(F)(X)$ sits in degrees $[0, \operatorname{dim} X]$.

Proof. (i) Since $\Xi^{n}(X)$ is directed, the functor $F \mapsto F_{\mathrm{Nis}}^{n}$ is exact, and $\mathcal{C}^{\mathrm{Nis}}(F)^{n}$ is a natural direct summand in $F_{\mathrm{Nis}}^{n}$.

(ii) By construction, the functor $F \mapsto F_{\mathrm{Nis}}^{n}$ is $n+1$-iteration of one $F \mapsto F_{\mathrm{Nis}}^{0}$ (i.e., $\left.F_{\mathrm{Nis}}^{n}=\left(F_{\mathrm{Nis}}^{n-1}\right)_{\mathrm{Nis}}^{0}\right)$. Replacing $F_{\mathrm{Nis}}^{n-1}$ by $F$, we see that it suffices to show that $F_{\mathrm{Nis}}^{0}$ is Nisnevich local. Indeed, for every Nisnevich hypercovering $V$. of $X$ the complex $F_{\mathrm{Nis}}^{0}(V . / X)$ is homotopically trivial, which follows easily from the existence of a constructible (left) contraction of $V . / X ;{ }^{23}$ the details are left to the reader.

(iii) Take any $X \in \mathcal{S} m$ and a point $x \in X$; let $Y:=X_{x}^{h}$ be the Henselization of $X$ at $x$ considered as a proobject of $\mathcal{S} m$. To check that $F(Y) \rightarrow C^{\mathrm{Nis}}(F)(Y)$ is a quasi-isomorphism, one shows that the augmented simplicial proobject $Y^{\mathrm{Nis}} / Y$ is contractible. The contraction $c$ is unique; its construction is left to the reader.

(iv) Let us define a cofinal subset $\Xi_{c}^{n}$ of $\Xi_{o}^{n}$ which clarifies the structure of $F_{\mathrm{Nis}}^{n}$.

Let $\left\{Z_{\alpha}\right\}$ be a stratification of $X$ with equidimensional strata. Its set $A$ of indices $\alpha$ is naturally ordered $\left(\alpha^{\prime} \geq \alpha\right.$ means $\left.\bar{Z}_{\alpha^{\prime}} \supset Z_{\alpha}\right)$, so we have the simplicial set $S(A)$. where $S(A)_{n}$ is the set of ordered $n+1$-tuples $\left(\alpha_{0} \ldots \alpha_{n}\right), \alpha_{0} \leq \ldots \leq \alpha_{n}$.

For $\xi \in \Xi(X)$ we denote by $\left\{Z_{\alpha}^{\xi}\right\}$ the induced stratification of $U^{\xi}$, and by $U_{\alpha}^{\xi}$ the union of connected components of $U^{\xi}$ that contains $s^{\xi}\left(Z_{\alpha}\right)$. We say that $\xi$ is

\footnotetext{
${ }^{23}$ Recall that a left contraction of an augmented simplicial object $V$. $/ X$ is a datum $c$. of sections $c_{n}: V_{n} \rightarrow V_{n+1}$ of $\partial_{0}: V_{n+1} \rightarrow V_{n}, n \geq-1$ (here $V_{-1}:=X$ ), compatible with the simplicial structure maps, in the sense that for every monotone $\phi:[0, n] \rightarrow[0, m]$ one has $c_{n} \phi_{V}=\tilde{\phi}_{V} c_{m}$ : $V_{m} \rightarrow V_{n+1} ;$ here $\tilde{\phi}:[0, n+1] \rightarrow[0, m+1]$ is defined as $\tilde{\phi}(0)=0, \tilde{\phi}(a)=\phi(a-1)+1$ for $a \in[1, n+1]$.
} 
$\left\{Z_{\alpha}\right\}$-controlled if $U^{\xi}$ equals the disjoint union of $U_{\alpha}^{\xi}$, for each $\alpha$ the intersection $Z_{\alpha^{\prime}}^{\xi} \cap U_{\alpha}^{\xi}$ is empty unless $\alpha^{\prime} \geq \alpha$, and $Z_{\alpha}^{\xi} \cap U_{\alpha}^{\xi} \stackrel{\sim}{\rightarrow} Z_{\alpha}$. Notice that $\left.s^{\xi}\right|_{Z_{\alpha}}$ is the inverse to the latter isomorphism.

We say that $\xi \in \Xi^{n}(X)$ is $\left\{Z_{\alpha}\right\}$-controlled if each $\xi_{i} \in \Xi\left(U^{\xi_{i-1}}\right)$ is $\left\{Z_{\alpha}^{\xi_{i-1}}\right\}$ controlled; here $\left\{Z_{\alpha}^{\xi_{i-1}}\right\}$ is the stratification of $U^{\xi_{i-1}}$ induced by $\left\{Z_{\alpha}\right\}$. Then every $U^{\xi_{m}}, m \in[0, n]$, is the disjoint union of components $U_{\alpha_{0} \ldots \alpha_{m}}^{\xi_{m}}$ labeled by $\left(\alpha_{0} \ldots \alpha_{m}\right)$ $\in S(A)_{m}$, defined as the intersection of the preimages of $U_{\alpha_{i}}^{\xi_{i}} \subset U^{\xi_{i}}$ for $i \leq m$.

Suppose $\xi \in \Xi^{n}(X), n>0$, is $\left\{Z_{\alpha}\right\}$-controlled. For $m \in[0, n-1]$ let $\sigma_{m}$ : $[0, n] \rightarrow[0, n-1]$ be the monotone surjection with $\sigma_{m}(m)=\sigma_{m}(m+1)$. Then $\xi^{\sigma_{m}} \in \Xi^{n-1}(X)$ need not be $\left\{Z_{\alpha}\right\}$-controlled, but there is a natural morphism $\bar{\xi}^{\sigma_{m}} \rightarrow \xi^{\sigma_{m}}$ in $\Xi^{n-1}(X)$ with $\left\{Z_{\alpha}\right\}$-controlled $\bar{\xi}^{\sigma_{m}}$. Namely, $U^{\bar{\xi}_{i}^{\sigma}}$ equals $U^{\xi_{i}^{\sigma m}}$ if $i \leq m$; otherwise $U^{\bar{\xi}_{i}^{\sigma}}$ is an open subset of $U^{\xi_{i}^{\sigma_{m}}}=U^{\xi_{i+1}}$ equal to the union of all components $U_{\alpha_{0} \ldots \alpha_{i+1}}^{\xi_{i+1}}$ with $\alpha_{m}=\alpha_{m+1}$.

Lemma. $\xi$ 's in $\Xi^{n}(X)$ that are controlled by some stratification form a cofinal subset $\Xi_{c}^{n}(X)$ in $\Xi_{o}^{n}(X)$. If $n>0$, then for every $m \in[0, n]$ all $\bar{\xi}^{\sigma_{m}}, \xi \in \Xi_{c}^{n}(X)$, form a cofinal subset in $\Xi_{c}^{n-1}(X)$.

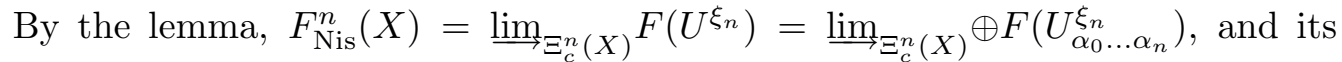
submodule $\mathcal{C}^{\mathrm{Nis}}(F)^{n}(X)$ is the inductive limit of the sum of components $F\left(U_{\alpha_{0} \ldots \alpha_{n}}^{\xi_{n}}\right)$ with $\alpha_{0}<\ldots<\alpha_{n}$. Since the image of $F\left(U_{\alpha_{0} \ldots \alpha_{n}}^{\xi_{n}}\right)$ in $F_{\mathrm{Nis}}^{n}(X)$ vanishes on the complement of $\bar{Z}_{\alpha_{0}}$, we are done with the proposition.

For a complex of presheaves $F$ we denote by $\mathcal{C}^{\mathrm{Nis}}(F)$ the corresponding total complex. We see that $\mathcal{C}^{\mathrm{Nis}}$ is a DG endofunctor that lifts the Nisnevich localization.

4.3. Compatibility with transfers. The Nisnevich topology, as opposed to the Zariski one, is correspondence friendly:

Proposition. The Nisnevich coverings define a Grothendieck topology on $R_{\mathrm{tr}}[\mathcal{S} m]$.

Proof. For a smooth $X$ each Nisnevich cover $U / X$ yields a sieve on $R_{\mathrm{tr}}[X]$ formed by those correspondences $\operatorname{Cor}(\cdot, X)$ that factor through $U$. The axioms of Grothendieck topology (see e.g. [D1] Arcata I 6) are immediate, except the one which is the next lemma for $X, Y$ smooth:

Lemma. Let $X, Y$ be schemes, and $\pi: U \rightarrow X$ be a Nisnevich cover; suppose that $Y$ is normal. Then for any $\gamma \in \mathcal{C}$ or $(Y, X)$ one can find a Nisnevich cover $\pi^{\prime}: V \rightarrow Y$ and $\tilde{\gamma} \in \mathcal{C}$ or $(V, U)$ such that $\pi \tilde{\gamma}=\gamma \pi^{\prime}$.

Proof of Lemma. Those $\gamma$ for which the assertion is true form an $R$-submodule of $\mathcal{C}$ or $(Y, X)$. Indeed, if $\gamma=\Sigma a_{i} \gamma_{i}, a_{i} \in R$, and we have found $V_{i} / Y$ and $\tilde{\gamma}_{i}$ for each $\gamma_{i}$, then we take for $V / Y$ the fiber product of $V_{i}$ 's over $Y$, and define $\tilde{\gamma}$ as $\Sigma a_{i} \tilde{\gamma}_{i}^{\prime}$, where $\tilde{\gamma}_{i}^{\prime}$ is the composition of $\tilde{\gamma}_{i}$ and the projection $V \rightarrow V_{i}$.

Thus it suffices to consider the case of $\gamma$ given by a reduced irreducible $\Gamma \subset X \times Y$. Consider the Nisnevich cover $U_{\Gamma}:=U \underset{X}{\times} \Gamma$ of $\Gamma$. Since $\Gamma$ is finite over $Y$, one can find a Nisnevich cover $\pi^{\prime}: V \rightarrow Y$ together with a morphism of $\Gamma$-schemes 
$\theta: V_{\Gamma}:=V \underset{Y}{\times} \Gamma \rightarrow U_{\Gamma}$. Take for $\tilde{\gamma}$ the composition $V \rightarrow V_{\Gamma} \stackrel{\theta}{\longrightarrow} U_{\Gamma} \rightarrow U$ where the first arrow is the finite correspondence transposed to the projection $V_{\Gamma} \rightarrow V$.

A Nisnevich sheaf with transfers is a presheaf with transfers which is a Nisnevich sheaf. Equivalently, this is an $R$-sheaf $F$ on the topology from the lemma such that for every $X, Y \in R_{\mathrm{tr}}[\mathcal{S} m]$ the pull-back map $\operatorname{Cor}(Y, X) \times F(X) \rightarrow F(Y)$ is $R$-bilinear. These objects form an abelian category $\mathcal{S} h_{\mathrm{tr}}^{\mathrm{Nis}}$, and we have the exact sheafification functor $\mathcal{P} \mathcal{S} h_{\mathrm{tr}} \rightarrow \mathcal{S} h_{\mathrm{tr}}^{\mathrm{Nis}}, F \mapsto F_{\mathrm{Nis}}$, left adjoint to the fully faithful embedding $\mathcal{S} h_{\mathrm{tr}}^{\mathrm{Nis}} \hookrightarrow \mathcal{P} \mathcal{S} h_{\mathrm{tr}}$. The forgetful functors $o: \mathcal{P} \mathcal{S} h_{\mathrm{tr}} \rightarrow \mathcal{P} \mathcal{S}$, $\mathcal{S} h_{\mathrm{tr}}^{\mathrm{Nis}} \rightarrow \mathcal{S} h^{\mathrm{Nis}}$ commute with the sheafification and the embedding.

Let $I_{\mathrm{tr}}^{\mathrm{Nis}}$ be the triangulated subcategory of $D \mathcal{P} \mathcal{S} h_{\mathrm{tr}}$ generated by all Nisnevich Mayer-Vietoris complexes $R_{\mathrm{tr}}\left[U_{V}\right] \rightarrow R_{\mathrm{tr}}[U] \oplus R_{\mathrm{tr}}[V] \rightarrow R_{\mathrm{tr}}[X]$ (see 4.2.1). It is right admissible (by (i) of the proposition from 1.4.2). The forgetful functor $o: D \mathcal{P S} h_{\mathrm{tr}} \rightarrow D \mathcal{P S} h$ sends $I_{\mathrm{tr}}^{\mathrm{Nis}}$ to $I^{\mathrm{Nis}}$ (by the lemma) and $I_{\mathrm{tr}}^{\mathrm{Nis} \perp}$ to $I^{\mathrm{Nis} \perp}$ (by the proposition in 4.2.1), i.e., o commutes with the Nisnevich localization $\mathcal{C}^{\mathrm{Nis}}$. Since $O$ is conservative, $I_{\mathrm{tr}}^{\mathrm{Nis}}, I_{\mathrm{tr}}^{\mathrm{Nis} \perp}$ are formed exactly by those complexes of presheaves with transfers that are Nisnevich locally acyclic, resp. Nisnevich local, as mere complexes of presheaves. So the Nisnevich sheafification yields equivalences

$$
D \mathcal{P S} h_{\mathrm{tr}} / I_{\mathrm{tr}}^{\mathrm{Nis}} \stackrel{\sim}{\rightarrow} D \mathcal{S} h_{\mathrm{tr}}^{\mathrm{Nis}} \stackrel{\sim}{\leftarrow} I_{\mathrm{tr}}^{\mathrm{Nis} \perp} .
$$

The t-structure picture here is that of (i) from the lemma in 1.2.

The canonical resolution $\mathcal{C}^{\mathrm{Nis}}$ from 4.2 .2 is compatible with transfers. Namely, any $X \in \mathcal{S} m$ yields a simplicial proobject $R_{\mathrm{tr}}\left[X^{\mathrm{Nis}}\right]$ in $\mathcal{C}$ or $(\mathcal{S} m)$ (see 4.2.2), and, as in the lemma above, any $\gamma \in \mathcal{C} \operatorname{Cor}(Y, X)$ lifts naturally to a morphism $R_{\mathrm{tr}}\left[Y^{\mathrm{Nis}}\right] \rightarrow$ $R_{\mathrm{tr}}\left[X^{\mathrm{Nis}}\right]$. Therefore for any presheaf with transfers $F$ its resolution $\mathcal{C}^{\mathrm{Nis}}(F)$ has a canonical transfer structure, and the morphism $F \rightarrow \mathcal{C}^{\mathrm{Nis}}(F)$ is compatible with transfers. So the Nisnevich sheafification $F_{\mathrm{Nis}}=H^{0} \mathcal{C}^{\mathrm{Nis}}(F)$ has a natural transfer structure as well. We see that $\mathcal{C}^{\mathrm{Nis}}$ is a DG endofunctor of $C \mathcal{P} \mathcal{S} h_{\mathrm{tr}}$ that lifts the Nisnevich localization.

Remark. The usual Godement resolution [Go] for the Nisnevich topology is compatible with transfers as well.

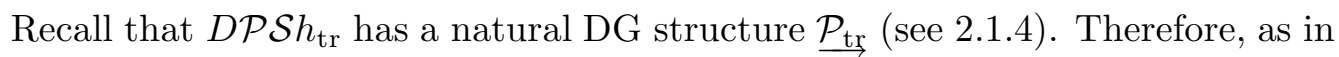
1.5.5, the DG subcategories $\stackrel{\mathcal{I}_{\mathrm{tr}}^{\mathrm{Nis}}}{\longrightarrow}, \stackrel{\mathcal{I}_{\mathrm{tr}}^{\mathrm{Nis}} \perp}{\longrightarrow} \subset \underset{\mathcal{P}_{\mathrm{tr}}}{\longrightarrow}$ of objects that belong to $I_{\mathrm{tr}}^{\mathrm{Nis}}, I_{\mathrm{tr}}^{\mathrm{Nis} \perp} \subset$ $D \mathcal{P} \mathcal{S} h_{\mathrm{tr}}=\mathcal{P}_{\mathrm{tr}}^{\mathrm{tri}}$, are DG structures on $I_{\mathrm{tr}}^{\mathrm{Nis}}, I_{\mathrm{tr}}^{\mathrm{Nis} \perp}$, and one has a quasi-equivalence of DG categories $\stackrel{\mathcal{I}_{\mathrm{tr}}^{\mathrm{Nis}} \perp}{\longrightarrow} \stackrel{\sim}{\longrightarrow} \underset{\mathcal{P}_{\mathrm{tr}}}{\longrightarrow} / \stackrel{\mathcal{I}_{\mathrm{tr}}^{\mathrm{Ni}}}{\longrightarrow}$.

4.4. The motivic localization. Set $\mathcal{I}_{\mathrm{tr}}^{\mathrm{Nis}}:=\left(\underset{\mathcal{I}_{\mathrm{tr}}^{\mathrm{Nis}}}{\longrightarrow}\right)^{\text {perf }} \subset \mathcal{P}_{\mathrm{tr}}$, i.e., $\mathcal{I}_{\mathrm{tr}}^{\mathrm{Nis}}$ is the homotopy idempotent completion of the full pretriangulated DG subcategory strongly generated by the Nisnevich Mayer-Vietoris complexes. As in 2.3, let $\mathcal{I}_{\mathrm{tr}}^{\Delta \mathrm{Nis}} \subset \mathcal{P}_{\mathrm{tr}}$ be the corresponding subcategory strongly generated by $\mathcal{I}_{\mathrm{tr}}^{\Delta}$ and $\mathcal{I}_{\mathrm{tr}}^{\mathrm{Nis}}$. Then $I_{\mathrm{tr}}^{\Delta \mathrm{Nis}}:=\left(\mathcal{I}_{\mathrm{tr}}^{\Delta \mathrm{Nis}}\right)^{\mathrm{tri}}$ is the full triangulated subcategory of $D \mathcal{P} \mathcal{S} h_{\mathrm{tr}}$ generated by $I_{\mathrm{tr}}^{\mathrm{Nis}}$ and $I_{\mathrm{tr}}^{\Delta}$.

Theorem. Suppose that the base field $k$ is perfect.

(i) The subcategories $I_{\mathrm{tr}}^{\Delta}, I_{\mathrm{tr}}^{\mathrm{Nis}} \subset D \mathcal{P S} h_{\mathrm{tr}}$ are compatible (see 1.3). 
(ii) One has $I_{\mathrm{tr}}^{\Delta \mathrm{Zar}}=I_{\mathrm{tr}}^{\Delta \mathrm{Nis}}$ (see 2.3), i.e.,

$$
\begin{aligned}
& \underline{\mathcal{D}}_{\mathcal{M}}^{\text {eff tri }} \stackrel{\sim}{\rightarrow} D \mathcal{P} \mathcal{S} h_{\mathrm{tr}} / I_{\mathrm{tr}}^{\Delta \mathrm{Nis}}=I_{\mathrm{tr}}^{\Delta \perp} \cap I_{\mathrm{tr}}^{\mathrm{Nis} \perp}, \\
& \mathcal{D}_{\mathcal{M}}^{\text {eff tri }} \stackrel{\sim}{\rightarrow}\left(D \mathcal{P} \mathcal{S} h_{\mathrm{tr}} / I_{\mathrm{tr}}^{\Delta \mathrm{Nis}}\right)^{\text {perf }}
\end{aligned}
$$

Therefore one has a quasi-equivalence of the DG categories $\mathcal{D}_{\mathcal{M}}^{\mathrm{eff}} \stackrel{\sim}{\rightarrow} \mathcal{P}_{\mathrm{tr}} /{ }^{\kappa} \mathcal{I}_{\mathrm{tr}}^{\Delta \mathrm{Nis}}$, and the motivic localization endofunctor $\mathcal{C}^{\mathcal{M}}$ of $D \mathcal{P S} h_{\mathrm{tr}}$ can be written as

$$
\mathcal{C}^{\mathcal{M}}=\mathcal{C}^{\Delta} \mathcal{C}^{\mathrm{Nis}}=\mathcal{C}^{\mathrm{Nis}} \mathcal{C}^{\Delta}
$$

Remarks. (a) For an interpretation of (i) on finite level (inside $\mathcal{P}_{\text {tr }}$ ), see 1.4.3.

(b) The theorem implies that in the definition of $\mathcal{D}_{\mathcal{M}}^{\text {eff }}$ (see 2.3) one can replace $\operatorname{Cor}(\mathcal{S} m)$ by its full subcategory of affine varieties: the category of motives does not change. We do not know if this remains true for non perfect $k$.

(c) We see that the identification $\underline{\mathcal{D}}_{\mathcal{M}}^{\text {eff tri }}=I_{\mathrm{tr}}^{\Delta \perp} / I_{\mathrm{tr}}^{\Delta \perp} \cap I_{\mathrm{tr}}^{\mathrm{Nis}}$ yields a t-structure on $\underline{\mathcal{D}}_{\mathcal{M}}^{\text {eff tri }}$ whose core is the abelian category of $\mathbb{A}^{1}$-homotopy invariant Nisnevich sheaves with transfers $\mathcal{S} h_{\mathrm{tr}}^{\Delta \mathrm{Nis}}$. To sum the picture up, we have a tensor DG category $\underset{\mathcal{P}_{\mathrm{tr}}}{\longrightarrow}$ and its three quotients. The corresponding homotopy categories carry nondegenerate t-structures with cores $\mathcal{P} h_{\mathrm{tr}}, \mathcal{S} h_{\mathrm{tr}}^{\mathrm{Nis}}, \mathcal{P} \mathcal{S} h_{\mathrm{tr}}^{\Delta}, \mathcal{S} h_{\mathrm{tr}}^{\Delta \mathrm{Nis}}$. The projections

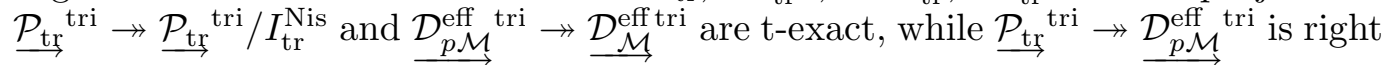
t-exact (its right adjoint is exact). The tensor products are right t-exact.

(d) We do not know if $\underline{\mathcal{D}}_{\mathcal{M}}^{\text {eff tri }}$ is equivalent to the derived category of $\mathcal{S} h_{\mathrm{tr}}^{\Delta \mathrm{Nis}}$ (cf. the exercise in 3.1.2).

Plan of the proof. First, we reformulate the theorem in concrete terms, reducing it to the theorem in 4.5. The latter is deduced in 4.6.4 from the (itself important) theory of residues for $\mathbb{A}^{1}$-homotopy invariant presheaves with transfers, which is the subject of 4.6. The proofs of the principal facts about residues (the proposition in 4.6.1 and the theorem in 4.6.3) and of the lemma in 4.6.4 are given in $\S 5$.

4.5. Theorem. If $k$ is perfect and $F \in \mathcal{P} \mathcal{S} h_{\mathrm{tr}}$ is $\mathbb{A}^{1}$-homotopy invariant, then for every $X \in \mathcal{S} m$ one has

$$
H_{\mathrm{Nis}}(X, F) \stackrel{\sim}{\rightarrow} H_{\mathrm{Nis}}\left(X \times \mathbb{A}^{1}, F\right), \quad H_{\text {Zar }}^{\cdot}(X, F) \stackrel{\sim}{\rightarrow} H_{\text {Nis }}(X, F)
$$

In particular, the Zariski and Nisnevich sheafifications of $F$ coincide: $F_{\mathrm{Zar}} \stackrel{\sim}{\rightarrow} F_{\mathrm{Nis}}$.

Here $H_{\mathrm{Nis}}^{a}(X, F):=H^{a}\left(X_{\mathrm{Nis}}, F_{\mathrm{Nis}}\right)=H^{a} \mathcal{C}^{\mathrm{Nis}}(F)(X)$, etc.

Remark. Since the small Zariski and Nisnevich topologies have finite cohomological dimension, the theorem automatically implies that (4.5.1) remains true for any complex $F \in I_{\mathrm{tr}}^{\Delta \perp}$.

Let us show that the above theorem implies the theorem in 4.4: 
(a) 4.4(i) is equivalent to the first isomorphism in (4.5.1). By 1.3(ii), 4.4(i) amounts to the fact that the Nisnevich localization $\mathcal{C}^{\mathrm{Nis}}$ preserves $I_{\mathrm{tr}}^{\Delta}$ and $I_{\mathrm{tr}}^{\Delta \perp}$.

The statement about $I_{\mathrm{tr}}^{\Delta}$ is easy (we need not assume 4.5, and make no use of transfers). Indeed, for any presheaf $F$ the canonical embedding $\mathcal{C}^{\mathrm{Nis}}(F) \hookrightarrow$ $\mathcal{C}^{\Delta}\left(\mathcal{C}^{\mathrm{Nis}}(F)\right)$ is the composition of natural embeddings $\mathcal{C}^{\mathrm{Nis}}(F) \hookrightarrow \mathcal{C}^{\mathrm{Nis}}\left(\mathcal{C}^{\Delta}(F)\right) \hookrightarrow$ $\mathcal{C}^{\Delta}\left(\mathcal{C}^{\mathrm{Nis}}(F)\right)$. Now if $F \in I_{\mathrm{tr}}^{\Delta}$, then $\mathcal{C}^{\Delta}(F)$ is acyclic, hence the morphism $\mathcal{C}^{\mathrm{Nis}}(F) \hookrightarrow$ $\mathcal{C}^{\Delta}\left(\mathcal{C}^{\mathrm{Nis}}(F)\right)$ vanishes in $D \mathcal{P} \mathcal{S} h$, which implies that $\mathcal{C}^{\mathrm{Nis}}(F) \in I_{\mathrm{tr}}^{\Delta}$.

The statement about $I_{\mathrm{tr}}^{\Delta \perp}$ says that for every $\mathbb{A}^{1}$-homotopy invariant complex of presheaves with transfers $F$ its Nisnevich localization $\mathcal{C}^{\mathrm{Nis}}(F)$ is again $\mathbb{A}^{1}$-homotopy invariant, i.e., for every $X \in \mathcal{S} m$ one has $H_{\text {Nis }}(X, F) \stackrel{\sim}{\rightarrow} H_{\text {Nis }}\left(X \times \mathbb{A}^{1}, F\right)$, which by the above Remark is the first isomorphism in (4.5.1), q.e.d.

(b) 4.4(ii) follows from the second isomorphism in (4.5.1). Indeed, 4.4(ii) amounts to the fact that $I_{\mathrm{tr}}^{\mathrm{Nis} \perp} \cap I_{\mathrm{tr}}^{\Delta \perp}=I_{\mathrm{tr}}^{\mathrm{Zar} \perp} \cap I_{\mathrm{tr}}^{\Delta \perp}$. Since $I_{\mathrm{tr}}^{\mathrm{Nis} \perp} \subset I_{\mathrm{tr}}^{\mathrm{Zar} \perp}$, this means that every $\mathbb{A}^{1}$-homotopy invariant complex of presheaves with transfers which is Zariski local (as a mere complex of presheaves) is automatically Nisnevich local. It suffices to check that for every $F \in I_{\mathrm{tr}}^{\Delta \perp}$ its Nisnevich localization coincides, as an object of $D \mathcal{P S} h$, with the Zariski localization $F^{\mathrm{Zar}}$ of $F$ (in the sense of mere presheaves), ${ }^{24}$ which by the above Remark is the second isomorphism in (4.5.1), q.e.d.

Exercise (Gaitsgory). The second isomorphism in (4.5.1) is equivalent to 4.4(ii) combined with the fact that $o: D \mathcal{P} \mathcal{S} h_{\mathrm{tr}} \rightarrow D \mathcal{P} \mathcal{S} h$ sends $I_{\mathrm{tr}}^{\Delta \perp} \cap I_{\mathrm{tr}}^{\mathrm{Zar}}$ to $I^{\text {Zar }}$.

The next corollary of the theorems in 4.4, 4.5 captures their essence:

Corollary. If $Y$ is a smooth local scheme, ${ }^{25}$ e.g. $Y=$ Speck, then the functor $X$ $\mapsto \mathcal{C}^{\Delta}\left(R_{\mathrm{tr}}[X]\right)(Y)$ has Nisnevich local nature, i.e., for any Nisnevich hypercovering $U$. of $X$ the map $\mathcal{C}^{\Delta}\left(R_{\mathrm{tr}}[U].\right)(Y) \rightarrow \mathcal{C}^{\Delta}\left(R_{\mathrm{tr}}[X]\right)(Y)$ is a quasi-isomorphism.

4.6. Residues and the Cousin resolution. 4.6.1. We use the notation of 3.4. Let $X$ be a smooth variety, $Z \subset X$ a smooth divisor, i.e., we have a smooth pair $(X, X \backslash Z)$.

Definition. $\theta \in \mathcal{C} \operatorname{Cor}(Z \times \mathbb{T},(X, X \backslash Z))$ is a link correspondence (for $(X, X \backslash Z)$, or simply at $Z)$ if $S p(\theta) \in \mathcal{C}$ or $(Z, Z)$ equals $\operatorname{id}_{Z}$.

Using the homotopy equivalence $R_{\mathrm{tr}}^{\Delta}\left[Z \times \mathbb{G}_{m} / Z \times \mathbb{A}^{1}\right] \stackrel{\sim}{\rightarrow} R_{\mathrm{tr}}^{\Delta}[Z](1)[1]$, we can rewrite $\theta^{\text {rel }}$ as a morphism in the homotopy category of premotives

$$
\theta^{\text {rel }}: R_{\mathrm{tr}}^{\Delta}[Z](1)[1] \rightarrow R_{\mathrm{tr}}^{\Delta}[(X \backslash Z) / X]
$$

Proposition. Let $X, Z$ be as above, and $P \subset Z$ be a finite subset of points. Suppose that the base field $k$ is perfect and $X$ is quasi-projective.

(i) There is a Zariski neighborhood $U \subset X$ of $P$ such that $(U, U \backslash Z)$ admits a link correspondence $\theta .^{26}$

\footnotetext{
${ }^{24}$ Hence, in particular, $F^{\text {Zar }}$ acquires a transfer structure (cf. 4.1).

${ }^{25}$ I.e., $Y$ is the localization of a smooth variety at a point. In fact, by (ii) of the theorem in 4.6.3, the statement is valid whenever $Y$ is a smooth semi-local scheme.

${ }^{26}$ For this statement $k$ need not be perfect: it suffices to demand that $P$ consists of smooth points (i.e., the generic points of smooth locally closed subschemes).
} 
(ii) For every such $U$ and link correspondences $\theta, \theta^{\prime}$ for $(U, U \backslash Z)$ one can find an open $Z^{\prime} \subset U \cap Z$ which contains $P$ such that the restrictions $R_{\mathrm{tr}}^{\Delta}\left[Z^{\prime}\right](1)[1] \rightarrow$ $R_{\mathrm{tr}}^{\Delta}[(U \backslash Z) / U]$ of $\theta^{\text {rel }}$, $\theta^{\prime r e l}$ to $Z^{\prime}$ are $\mathbb{A}^{1}$-homotopic.

For a proof, see 5.4.

Remarks. (a) It follows from 5.3 and 5.2 that $U$ and $\theta$ from (i) can be chosen so that $\theta^{r e l}$ is a homotopy equivalence.

(b) In 5.4 we prove the proposition under the additional assumption that $k$ is infinite. The case of finite $k$ follows then by the next trick. A standard argument (localizing $R$ at two different primes, etc.) shows that one can assume that certain prime $\ell$ is invertible in $R$. Let $k^{\prime}$ be a maximal pro- $\ell$-extension of $k$, $G:=\operatorname{Gal}\left(k^{\prime} / k\right) \simeq \mathbb{Z}_{\ell}$. For any $Y_{1}, Y_{2} \in \mathcal{S} m_{k}$ we have the $R$-module $\mathcal{C}$ or $\left(Y_{1 k^{\prime}}, Y_{2 k^{\prime}}\right){ }_{k^{\prime}}$ of correspondences between the $k^{\prime}$-varieties $Y_{1 k^{\prime}}, Y_{2 k^{\prime}}$; the group $G$ acts naturally on it, and $\operatorname{Cor}\left(Y_{1}, Y_{2}\right)=\mathcal{C} \operatorname{or}\left(Y_{1 k^{\prime}}, Y_{2 k^{\prime}}\right)_{k^{\prime}}^{G}$. Due to the condition on $R$, the functor of $G$ invariants is exact on $R[G]$-modules, so the existence of a link correspondence over $k^{\prime}$ implies that over $k$. Same is true if we consider $\mathbb{A}^{1}$-localized correspondences, so (ii) of the proposition for $k$ follows from that for $k^{\prime}$, q.e.d.

Question. Is it true that morphisms $\theta^{\text {rel }}$ for all link correspondences at $Z$ localized at $P$ form naturally a contractible space? That is, can one present, after the localization at $P$, a natural complex $C$ of $R$-modules with $H^{0} C=R, H^{\neq 0} C=0$, and a morphism $C \rightarrow \operatorname{Hom}\left(R_{\mathrm{tr}}^{\Delta}[Z](1)[1], R_{\mathrm{tr}}^{\Delta}[(X \backslash Z) / X]\right)$ whose image contains the $\theta^{\text {rel }}$ s?

4.6.2. Assuming the above proposition, let us define the residue maps.

Suppose that $k$ is perfect. This means that any reduced scheme $X$ of finite type has a smooth open dense subscheme. As in 2.1.1, for $F \in \mathcal{P S} h$ we have a welldefined abelian group $F\left(\eta_{X}\right)$; here $\eta_{X}$ is the union of the generic points of $X$.

Let $F$ be an $\mathbb{A}^{1}$-homotopy invariant presheaf with transfers. As in 2.2 , we have $F_{-n} \in \mathcal{P} \mathcal{S} h_{\mathrm{tr}}, n \geq 0$; they are all $\mathbb{A}^{1}$-homotopy invariant. ${ }^{27}$

Let $T$ be a semi-local reduced $k$-scheme of Krull dimension 1 which is localization of a $k$-scheme of finite type. Let $s=s_{T}$ be the (disjoint) union of closed points, $\eta=\eta_{T}:=T \backslash s$ the union of the generic points. Let us define the residue map

$$
\operatorname{Res}=\operatorname{Res}^{T}=\operatorname{Res}_{s}: F(\eta) \rightarrow F_{-1}(s) .
$$

If $T$ is regular, then $(T, s)$ is the localization of a pair $(X, Z)$, where $X$ is a smooth affine variety, $Z \subset X$ a smooth divisor, at the generic points of $Z$. Recall that $F_{-1}(Z)=\operatorname{Hom}\left(R_{\mathrm{tr}}^{\Delta}[Z](1)[1], F\right)$ (see 2.2). Replace $X$ by a base of Zariski neighborhoods of $s$; then the pull-back maps for morphisms (4.6.1) provide, by the proposition, a well-defined map $\operatorname{Coker}(F(T) \rightarrow F(\eta)) \rightarrow F_{-1}(s)$, which yields $\operatorname{Res}_{s}$.

Remark. Let $X, Z$ be as above. By the proposition, we have a natural morphism $\operatorname{Res}_{Z}: F(X \backslash Z) \rightarrow\left(F_{-1}\right)_{\text {Zar }}(Z)$ defined as the pull-back map for morphisms (4.6.1) on all possible opens of $Z$. For $\phi \in F(X \backslash Z)$ one has $\operatorname{Res}_{s}\left(\phi_{\eta}\right)=\left(\operatorname{Res}_{Z}(\phi)\right)_{s}$.

\footnotetext{
${ }^{27}$ In fact, all functors $\mathcal{H o m}(P, \cdot), P \in \mathcal{P}_{\mathrm{tr}}$, from 2.2 preserve $I_{\mathrm{tr}}^{\Delta \perp} \subset D \mathcal{P} \mathcal{S} h_{\mathrm{tr}}$.
} 
For arbitrary $T$, let $\tilde{T}$ be the normalization of $T$; then Res is the composition $F(\eta) \rightarrow F_{-1}\left(s_{\tilde{T}}\right) \rightarrow F_{-1}(s)$, where the first arrow is the residue map for $\tilde{T}$ and the second one is the transfer map for the finite projection $s_{\tilde{T}} \rightarrow s$.

4.6.3. As above, our $k$ is perfect. For $X \in \mathcal{S} m$ denote by $X^{(n)}$ the set of its points of codimension $n$. For $x \in X^{(n)}$ let $\eta_{x} \subset X$ be the corresponding generic point. For a presheaf $F$ set $\operatorname{Cous}(F)^{n}(X):=\underset{x \in X^{(n)}}{\oplus} F_{-n}\left(\eta_{x}\right)$.

A smooth morphism $f: Y \rightarrow X$ yields a pull-back map $f^{*}: \operatorname{Cous}(F)^{n}(X) \rightarrow$ $\operatorname{Cous}(F)^{n}(Y)$ which sends $F_{-n}\left(\eta_{x}\right)$ to $\oplus F_{-n}\left(\eta_{y}\right)$ where the $y$ are the generic points of $f^{-1}(x)$. If $F$ has a transfer structure and $g: Z \rightarrow X$ is any morphism in $\mathcal{S} m$ of codimension $m$, then for every $a$ such that $a, a+m \geq 0$ we have the trace map $g_{*}: \operatorname{Cous}\left(F_{-a-m}\right)^{n-m}(Z) \rightarrow \operatorname{Cous}\left(F_{-a}\right)^{n}(X)$ whose non-zero components are the transfers $F_{-a-n}\left(\eta_{z}\right) \rightarrow F_{-a-n}\left(\eta_{g(z)}\right)$ for $\eta_{z}$ finite over $\eta_{g(z)}$. The pull-back and the trace are compatible with morphisms of $F$ 's and with compositions of $f$ 's and $g$ 's; if $f, g$ are as above and $Y \stackrel{g^{\prime}}{\leftarrow} Y \underset{X}{\times} \underset{f^{\prime}}{\rightarrow} Z$ are the projections, then $f^{*} g_{*}=g_{*}^{\prime} f^{\prime *}$.

In particular, the pull-back maps for smooth morphisms make each $\operatorname{Cous}(F)^{n}$ a presheaf on the small smooth topology of any $X \in \mathcal{S} m$.

Lemma. Each Cous $(F)^{n}$ is a local presheaf for the small Nisnevich and Zariski topologies, ${ }^{28}$ hence $H_{\text {Nis }}^{>0}\left(X, \operatorname{Cous}(F)^{n}\right)=H_{\text {Zar }}^{>0}\left(X, \operatorname{Cous}(F)^{n}\right)=0$.

There is an evident morphism $F(X) \rightarrow \operatorname{Cous}(F)^{0}(X)$ compatible with the pullback maps for smooth morphisms of $X$ 's. By the lemma, it extends by adjunction to the morphisms of the sheafifications $F_{\mathrm{Zar}} \rightarrow F_{\mathrm{Nis}} \rightarrow \operatorname{Cous}(F)^{0}$.

Now suppose that $F$ is an $\mathbb{A}^{1}$-homotopy invariant presheaf with transfers. We define the Cousin differential

$$
d=d_{X}: \operatorname{Cous}(F)^{n}(X) \rightarrow \mathcal{C} \text { ous }(F)^{n+1}(X)
$$

as follows. Our $d$ is an $X^{(n)} \times X^{(n+1)}$-matrix whose entries are maps $d_{x y}: F_{-n}\left(\eta_{x}\right) \rightarrow$ $F_{-n-1}\left(\eta_{y}\right)$. If $y$ lies in the closure of $x$, then $d_{x y}:=\operatorname{Res}_{s}$ where $s=\eta_{Y}$ and $T$ is the localization of the closure of $x$ at $y$; otherwise $d_{x y}=0$. Since every $\nu \in F_{-n}\left(\eta_{x}\right)$ comes from an open subscheme of the closure of $x$, there is only finitely many $y$ 's such that $d_{x y}(\nu) \neq 0$, so $d$ is correctly defined.

Theorem. (i) One has $d^{2}=0$, so d makes Cous $(F)$ a complex. For smooth $f$ and proper $g$ as above the corresponding maps $f^{*}: \operatorname{Cous}(F)(X) \rightarrow \operatorname{Cous}(F)(Y)$ and $g_{*}: \operatorname{Cous}\left(F_{-a-m}\right)(Z)[-m] \rightarrow \operatorname{Cous}\left(F_{-a}\right)(X)$ are morphisms of complexes.

(ii) The morphism $F_{Z a r} \rightarrow$ Cous $(F)^{0}$ makes Cous $(F)$ a resolution of $F_{Z a r}$ as sheaves on the small Zariski topology. In fact, $\operatorname{Cous}(F)(\hat{X})$ is a resolution of $F(\hat{X})$ if $\hat{X}$ is any smooth semi-local scheme. ${ }^{29}$

For a proof, see 5.6.

\footnotetext{
${ }^{28}$ See 1.11; in fact, $\operatorname{Cous}(F)^{n}$ are flabby sheaves (see [SGA4] V 4.1 for the definition).

${ }^{29}$ Which means that $\hat{X}$ is localization of an affine smooth scheme at a finite subset of points.
} 
Remark. The last statement in (ii) explains why $F_{\text {Zar }}$ carries a transfer structure (avoiding the reference to the Nisnevich localization). Indeed, a correspondence sends a local scheme to a semi-local one. Since sections of $F_{\text {Zar }}$ on the latter are the same as those of $F$, we know how the correspondence acts on them.

4.6.4. We need the following auxiliary lemma (to be proved in 5.1.2):

Lemma. Let $F$ be an $\mathbb{A}^{1}$-homotopy invariant presheaf with transfers, $\eta$ be the generic point of a smooth variety. Then $F\left(\mathbb{A}_{\eta}^{1}\right) \stackrel{\sim}{\rightarrow} H_{\mathrm{Zar}}^{0}\left(\mathbb{A}_{\eta}^{1}, F\right), H_{\mathrm{Zar}}^{1}\left(\mathbb{A}_{\eta}^{1}, F\right)=0$.

Let us deduce theorem 4.5 from the above theorem and the lemma.

Of course, 4.6.3(ii) implies that $\operatorname{Cous}(F)$ is a resolution of $F_{\mathrm{Nis}}$ as sheaves on small Nisnevich topology as well, hence $F_{\mathrm{Zar}}=F_{\mathrm{Nis}}$. By 4.6.3(ii) and the lemma in 4.6.3, one has $R \Gamma\left(X_{\mathrm{Zar}}, F_{\mathrm{Zar}}\right)=\operatorname{Cous}(F)(X)=R \Gamma\left(X_{\mathrm{Nis}}, F_{\mathrm{Nis}}\right)$, so we have the second isomorphism in (4.5.1).

It remains to show that the pull-back map $p_{X}^{*}: \mathcal{C}$ ous $(F)(X) \rightarrow \operatorname{Cous}(F)\left(X \times \mathbb{A}^{1}\right)$ for the projection $p_{X}: X \times \mathbb{A}^{1} \rightarrow X$ is a quasi-isomorphism. Our complexes carry natural finite filtrations: on $\operatorname{Cous}(F)(X)$ this is the stupid filtration, and on $\operatorname{Cous}(F)\left(X \times \mathbb{A}^{1}\right)$ the filtration whose $m$ th term is formed by all $F_{-n}\left(\eta_{z}\right)$ 's such that $p_{X}(z)$ has codimension $\geq m$ in $X$. Then $p_{X}^{*}$ is compatible with the filtrations. We will check that $p_{X}^{*}$ is, in fact, a filtered quasi-isomorphism.

Indeed, by 4.6.3(i), gr $p_{X}^{*}$ is the direct sum of morphisms $p_{\eta_{x}}^{*}: F_{-n}\left(\eta_{x}\right) \rightarrow$ $\operatorname{Cous}\left(F_{-n}\right)\left(\mathbb{A}_{\eta_{x}}^{1}\right), x \in X^{(n)}$. Since $F_{-n}$ is $\mathbb{A}^{1}$-homotopy invariant, our assertion follows from 4.6.3(ii) and the above lemma.

4.6.5. Question. Let $\left\{Z_{i}\right\}$ be a smooth stratification of $X$. Can one, upon suitable refinement of $\left\{Z_{i}\right\}$, recover $M(X)$ by gluing $M\left(Z_{i}\right)\left(\operatorname{codim} Z_{i}\right)$ together using link correspondences and homotopies between them? Cf. Question in 4.6.1.

For example, let $Z \subset X$ be a smooth divisor which admits a link correspondence $\theta$. Set $U:=X \backslash Z$ and $R_{\mathrm{tr}}\left[U \cup_{\theta} Z\right]:=\mathcal{C}$ one $\left(R_{\mathrm{tr}}\left[Z \times \mathbb{G}_{m}\right] \rightarrow R_{\mathrm{tr}}[U] \oplus R_{\mathrm{tr}}\left[Z \times \mathbb{A}^{1}\right]\right)$, the arrow is the difference of $\theta$ and the embedding $Z \times \mathbb{G}_{m} \hookrightarrow Z \times \mathbb{A}^{1}$. One has a morphism $\alpha: R_{\mathrm{tr}}\left[U \cup_{\theta} Z\right] \rightarrow R_{\mathrm{tr}}[X]$ which is $U \hookrightarrow X$ on $R_{\mathrm{tr}}[U]$ and $\tilde{\theta}$ on $R_{\operatorname{tr}}\left[Z \times \mathbb{A}^{1}\right]$.

Exercise. Show that $\alpha$ is a homotopy equivalence in $\mathcal{D}_{\mathcal{M}}^{\text {eff }}{ }^{30}$

\section{$\S 5$. The residual proofs}

In this section we prove the assertions from 4.6. The material of 5.1 is a version of the contents of lectures 11, 21 in [MVW] and $\S \S 2,4.1-4.3$ of [Vo1], the proposition in 4.3 is M. Walker's theorem from [MVW] 11.17. The proof in 5.6 of the theorem from 4.6.3 is based on a variant of Gabber's argument [G].

5.1. Splitting off a puncture on a curve. Let $X$ be a smooth affine curve, $z$ a $k$-point of $X, U:=X \backslash\{z\}$. Suppose $z$ is a principal divisor, so we have $\varphi \in \mathcal{O}(X)$ with $\operatorname{div} \varphi=z$. Consider the morphism $R[U] \rightarrow R[X] \oplus R(1)[1]$ whose components come from the embedding $U \hookrightarrow X$ and the map $U \stackrel{\varphi}{\rightarrow} \mathbb{G}_{m}$ followed by a projector to $R(1)[1]$ (see 2.2). The gist of this section is the next assertion:

\footnotetext{
${ }^{30}$ Hint: use the proposition in 4.6 .1 and Remark (a) in loc. cit.
} 
$R_{\mathrm{tr}}^{\Delta}[U] \rightarrow R_{\mathrm{tr}}^{\Delta}[X] \oplus R_{\mathrm{tr}}^{\Delta}(1)[1]$ is a homotopy equivalence of premotives. The projection $R_{\mathrm{tr}}^{\Delta}[U] \rightarrow R_{\mathrm{tr}}^{\Delta}(1)[1]$ is left inverse to the composition $R_{\mathrm{tr}}^{\Delta}(1)[1] \rightarrow R_{\mathrm{tr}}^{\Delta}[U / X] \rightarrow$ $R_{\mathrm{tr}}^{\Delta}[U]$, where the first arrow is the map (4.6.1) coming from any link correspondence for $(X, z)$.

Below we consider a more general setting when we have a finite subset of points (instead of a single $z$ ), and the picture depends on parameters.

5.1.1. For us, a triple is a datum $(X / S, U)$ formed by smooth affine schemes $U$, $X$, and $S$, an open embedding $U \hookrightarrow X$, and a smooth morphism $X \rightarrow S$ of relative dimension 1, such that $Z:=X \backslash U$ is finite over $S$ (we consider $Z$ as a reduced scheme). A triple with properties (a), (b) below is called Voevodsky triple:

(a) The diagonal divisor $\Delta_{Z}: Z \hookrightarrow Z \underset{S}{\times} X^{\prime}$ is principal.

(b) There exists an open embedding of $S$-schemes $X \hookrightarrow \bar{X}$ such that $\bar{X}$ is normal and proper over $S, X^{\infty}:=\bar{X} \backslash X$ is finite over $S$, and $T:=X^{\infty} \sqcup Z$ admits a quasi-affine Zariski neighborhood in $\bar{X}{ }^{31}$

Notice that $\bar{X}$ is uniquely defined, $Z$ is a closed subscheme of $\bar{X}$, and $T$ is finite over $S$.

Proposition. (i) For a Voevodsky triple $(X / S, U)$ the morphism $R_{\mathrm{tr}}^{\Delta}[U] \rightarrow$ $R_{\mathrm{tr}}^{\Delta}[X]$ in is a homotopy split surjection, i.e., it admits a right inverse $s$ in $\mathcal{D}_{p \mathcal{M}}^{\text {eff tri }} .^{32}$ Therefore $R_{\mathrm{tr}}^{\Delta}[U / X] \rightarrow R_{\mathrm{tr}}^{\Delta}[U]$ is the kernel of this surjection.

(ii) For any $S$-scheme $Z / S$ the premotives $R_{\mathrm{tr}}^{\Delta}[U / X]$ for all Voevodsky triples $(X / S, U)$ with $Z=X \backslash U$ are canonically identified in the homotopy category $\mathcal{D}_{p \mathcal{M}}^{\text {eff tri }}$.

Proof. The promised splitting and identifications are produced by special correspondences defined as follows:

Let $(X / S, U),\left(X^{\prime} / S, U^{\prime}\right)$ be Voevodsky triples with the same $Z / S$. A meromorphic function $f$ on $X \underset{S}{\times} X^{\prime}$ is said to be special if for some open neighborhood $W$ of $T$ in $\bar{X}$ (see (b) above) our $f$ is a regular function on $W \underset{S}{\times} X^{\prime}$ whose restriction to $Z \underset{S}{\times} X^{\prime}$ is an equation of the divisor $\Delta_{Z}$ (see (a)), and $f$ equals 1 on $X^{\infty} \underset{S}{\times} X^{\prime}$. Such an $f$ exists due to (a): indeed, if $W \subset \bar{X}$ is any quasi-affine neighborhood of $T$, then every function on $T \underset{S}{\times} X^{\prime}$ extends to $W \underset{S}{\times} X^{\prime} .{ }^{33}$ Then $\operatorname{div}(f) \subset X \underset{S}{\times} X$ is finite over $X^{\prime}$, i.e., $\theta_{f}:=\operatorname{div}(f) \in \mathcal{C} \operatorname{or}\left(X^{\prime}, X\right)$. We call such a correspondence $S$-special, or simply special.

If the two triples coincide, then one can demand that $f$, in addition, vanishes on the diagonal $\Delta(W \cap X) \subset W \times X$. Such an $f$ exists due to (a), since every function

\footnotetext{
${ }^{31}$ The datum of (b) is essentially the same as standard triple from [Vo1] 4.1, [MVW] 11.5.

${ }^{32}$ Notice that $\mathcal{C}^{\Delta}\left(R_{\operatorname{tr}}[U]\right) \rightarrow \mathcal{C}^{\Delta}\left(R_{\operatorname{tr}}[X]\right)$ is injective as a morphism of complexes, yet it is a split surjection as a morphism in the homotopy category.

${ }^{33}$ Let us check the last assertion. Since $S$ is affine, $\operatorname{Spec} \mathcal{O}(W)$ is an $S$-scheme. Consider the embeddings $T \hookrightarrow W \hookrightarrow \operatorname{Spec} \mathcal{O}(W)$. Since $T$ is finite over $S$, it is a closed subscheme of $\operatorname{Spec} \mathcal{O}(W)$, hence $T \underset{S}{\times} X^{\prime} \hookrightarrow \operatorname{Spec} \mathcal{O}(W) \underset{S}{\times} X^{\prime}$ is a closed embedding of affine schemes, q.e.d.
} 
on $T \underset{S}{\times} X$ that vanishes on $\Delta_{Z}$ extends to a function on $\underset{S}{\underset{S}{\times}} X$ that vanishes on $\Delta(W \cap X) .{ }^{34}$ We refer then to $\theta_{f}$ as a very special correspondence.

Notice that for a special correspondence $\theta$ its restriction $\theta^{\circ}$ to $U^{\prime}$ lies in $\mathcal{C}$ or $\left(U^{\prime}, U\right)$, i.e., $\theta \in \mathcal{C}$ or $\left(\left(X^{\prime}, U^{\prime}\right),(X, U)\right)$ (see 3.4.1), so we have $\theta^{r e l}: R_{\mathrm{tr}}\left[U^{\prime} / X^{\prime}\right] \rightarrow R_{\mathrm{tr}}[U / X]$.

Lemma. (i) Any special $\theta$ is $\mathbb{A}^{1}$-homotopic to zero in $\mathcal{C}$ or $\left(X^{\prime}, X\right)$.

(ii) If $\theta_{1}, \theta_{2} \in \mathcal{C}$ or $\left(X^{\prime}, X\right)$ are special, then $\theta_{1}-\theta_{2} \in \mathcal{C}$ or $\left(X^{\prime}, U\right)$.

(iii) The composition of special correspondences is special.

Proof of Lemma. (i) Use a homotopy $\operatorname{div}(1-t+t f) \in \mathcal{C}$ or $\left(X^{\prime} \times \mathbb{A}^{1}, X\right)$.

(ii) Notice that $f_{1} / f_{2}$ is invertible on $Z \times X^{\prime}$.

(iii) Let $\left(X^{\prime \prime} / S, U^{\prime \prime}\right)$ be another Voevodsky triple with same $Z, \theta_{f^{\prime}} \in \mathcal{C}$ or $\left(X^{\prime \prime}, X^{\prime}\right)$ a special correspondence. Then $\theta_{f} \theta_{f^{\prime}}=\theta_{g}$ where $g\left(x, x^{\prime \prime}\right)=f\left(x, \theta_{f^{\prime}}\left(x^{\prime \prime}\right)\right)$ (here $\left.\left(x, x^{\prime \prime}\right) \in X \times X\right)$. Let us check that $g$ is special. Our $f$ is invertible on $T \times\left(X^{\prime} \backslash W^{\prime}\right)$ and $X^{\prime} \backslash \stackrel{S}{W^{\prime}}$ is finite over $S$, so there is an open neighborhood $W_{0} \subset \stackrel{S}{W}$ of $T$ such that $f$ is invertible on $W \underset{S}{\times}\left(X^{\prime} \backslash W^{\prime}\right)$. Then $g$ is regular on $W_{0} \underset{S}{\times} X^{\prime \prime}$. The rest is left to the reader.

We return to the proof of the proposition. (i) If $\theta \in \mathcal{C}$ or $(X, X)$ is very special, then $s:=\mathrm{id}_{X}-\theta$ lies in $\mathcal{C}$ or $(X, U)$. By (i) of the lemma, it is right inverse to $R_{\mathrm{tr}}^{\Delta}[U] \rightarrow R_{\mathrm{tr}}^{\Delta}[X]$ in the homotopy category, and we are done. Notice that the corresponding projector of $R_{\mathrm{tr}}^{\Delta}[U]$ onto the kernel of $R_{\mathrm{tr}}^{\Delta}[U] \rightarrow R_{\mathrm{tr}}^{\Delta}[X]$ equals the restriction $\theta^{o}$ of $\theta$ to $U$. Thus $\theta^{\text {rel }} \in \operatorname{End}\left(R_{\mathrm{tr}}^{\Delta}[U / X]\right)$ is homotopic to the identity.

(ii) By (ii) of the lemma, the composition $R_{\mathrm{tr}}^{\Delta}\left[U^{\prime} / X^{\prime}\right] \stackrel{\theta_{1}-\theta_{2}}{\longrightarrow} R_{\mathrm{tr}}^{\Delta}[U / X] \rightarrow R_{\mathrm{tr}}^{\Delta}[U]$ vanishes. Since, by (i), $R_{\mathrm{tr}}^{\Delta}[U / X] \rightarrow R_{\mathrm{tr}}^{\Delta}[U]$ is injective in the homotopy category, the morphism $\theta^{\text {rel }}: R_{\mathrm{tr}}^{\Delta}\left[U^{\prime} / X^{\prime}\right] \rightarrow R_{\mathrm{tr}}^{\Delta}[U / X]$ in the homotopy category does not depend on the choice of special $\theta$. This system of canonical morphisms

$$
R_{\mathrm{tr}}\left[U^{\prime} / X^{\prime}\right] \rightarrow R_{\mathrm{tr}}[U / X]
$$

in the homotopy category is transitive by (iii) of the lemma. Since $\theta^{\text {rel }}$ for $(X, U)=$ $\left(X^{\prime}, U^{\prime}\right)$ equals identity, (5.1.1) is a transitive system of isomorphisms, q.e.d.

Remarks. (i) Voevodsky triples are stable with respect to the base change by any $S^{\prime} \rightarrow S$ where $S^{\prime}$ is affine and smooth. The splittings and projectors constructed are compatible with the base change.

(ii) If $Z$ is smooth, then for any $S$-special correspondence $\theta$ one has $S p(\theta)=\mathrm{id}_{Z}$.

5.1.2. By 3.1.2, the above results translate into statements about $\mathbb{A}^{1}$-homotopy invariant presheaves with transfers. For example, we have the next proposition which implies the lemma in 4.6.4:

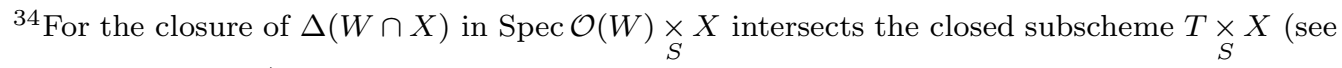
the previous footnote) by $\Delta_{Z}$.
} 
Proposition. Let $F$ be an $\mathbb{A}^{1}$-homotopy invariant presheaf with transfers. Let $\eta$ be the generic point of a smooth variety and $X \subset \mathbb{A}_{\eta}^{1}$ an open subscheme. Then $F(X) \stackrel{\sim}{\rightarrow} F_{\mathrm{Zar}}(X) \stackrel{\sim}{\rightarrow} F_{\mathrm{Nis}}(X)$ and $H_{\mathrm{Zar}}^{>0}\left(X, F_{\mathrm{Zar}}\right)=H_{\mathrm{Nis}}^{>0}\left(X, F_{\mathrm{Nis}}\right)=0$.

Proof. For every open $U \subset X$ the datum $(X / S, U)$ where $S=\eta$, is a Voevodsky triple. If $Z:=X \backslash U=\left\{z_{i}\right\}$, then, by (i) of the proposition in 5.1.1, the sequence $R_{\mathrm{tr}}^{\Delta}[U / X] \rightarrow R_{\mathrm{tr}}^{\Delta}[U] \rightarrow R_{\mathrm{tr}}^{\Delta}[X]$ in $\mathcal{D}_{p \mathcal{M}}^{\text {eff tri }}$ is split exact. Thus, in particular, the map $F(X) \rightarrow F(U)$ is injective.

Let $\pi: X^{\prime} \rightarrow X$ be any étale map such that $\pi^{-1}(Z) \stackrel{\sim}{\rightarrow} Z$. Then $\left(X^{\prime} / S, U^{\prime}\right)$, $U^{\prime}:=\pi^{-1}(U)$, is a Voevodsky triple, and for every $a \in X(k) \backslash Z$ the correspondence $\theta:=\pi-n \cdot a \in \operatorname{Cor}\left(X^{\prime}, X\right)$, where $a: X^{\prime} \rightarrow X$ is a constant map with value $a$, $n$ the degree of $Z$, is special. Notice that $\theta^{\text {rel }}: R_{\mathrm{tr}}^{\Delta}\left[U^{\prime} / X^{\prime}\right] \rightarrow R_{\mathrm{tr}}[U / X]$ equals $\pi$. Since $\theta^{r e l}$ is a homotopy equivalence by (the proof of) (ii) in the proposition in 5.1.1, one has $F(U) / F(X) \stackrel{\sim}{\rightarrow} F\left(U^{\prime}\right) / F\left(X^{\prime}\right)$. Passing to the inductive limit by all such $X^{\prime}$, resp. by those $X^{\prime}$ that are open subsets of $X$, we see that $F(U) / F(X) \stackrel{\sim}{\rightarrow}$ $H_{Z}^{1}\left(X_{\mathrm{Zar}}, F_{\mathrm{Zar}}\right) \stackrel{\sim}{\rightarrow} H_{Z}^{1}\left(X_{\mathrm{Nis}}, F_{\mathrm{Nis}}\right)$. This implies the assertion.

5.2. Nice pairs. We use the notation from 3.4.1. A Voevodsky triple $(X / S, U)$ is said to be nice if $Z:=X \backslash U$ is étale over $S$. A Voevodsky pair is a pair $(X, U)$ (see 3.4.1) which can be included in a Voevodsky triple $(X / S, U)$. A nice Voevodsky pair, or simply nice pair, is one that can be included in a nice $(X / S, U)$. We denote by $\operatorname{Cor}\left(\mathcal{P} a^{\text {nice }}\right)$ the full subcategory of $\operatorname{Cor}(\mathcal{P} a)$ formed by nice pairs. Notice that for any affine smooth $Z$ the pair $Z \times \mathbb{T}$ is nice.

Proposition. (i) For every Voevodsky pair $(X, U)$ the morphism $R_{\mathrm{tr}}^{\Delta}[U] \rightarrow$ $R_{\mathrm{tr}}^{\Delta}[X]$ is a split surjection in $\mathcal{D}_{p \mathcal{M}}^{\text {eff tri }}$ with kernel $R_{\mathrm{tr}}^{\Delta}[U / X] \rightarrow R_{\mathrm{tr}}^{\Delta}[U]$.

(ii) Every nice pair $(X, U)$ admits a link correspondence (see 4.6.1).

(iii) For every nice pair $(X, U)$ there is a canonical isomorphism

$$
R_{\mathrm{tr}}^{\Delta}[Z](1)[1] \stackrel{\sim}{\rightarrow} R_{\mathrm{tr}}^{\Delta}[U / X]
$$

in $\mathcal{D}_{p \mathcal{M}}^{\text {eff tri }}$ which is the identity map for $(X, U)=Z \times \mathbb{T}$. For every correspondence $\theta:\left(X^{\prime}, U^{\prime}\right) \rightarrow(X, U)$ between nice pairs it identifies $\theta^{\text {rel }}: R_{\mathrm{tr}}^{\Delta}\left[U^{\prime} / X^{\prime}\right] \rightarrow R_{\mathrm{tr}}^{\Delta}[U / X]$ with $S p(\theta)(1)$.

Proof. (i) is the same as (i) of the proposition from 5.1.1.

(ii) Since for a nice Voevodsky triple $(X / S, U)$ the triple $\left(Z \times \mathbb{A}^{1} / S, / Z \times \mathbb{G}_{m}\right)$ is a (nice) Voevodsky triple as well, there is an $S$-special $\theta \in \mathcal{C}$ or $(Z \times \mathbb{T},(X, U)$ ) (see the proof in 5.1.1). This is a link correspondence by Remark (ii) in 5.1.1.

(iii) It suffices to provide for each nice $(X, U)$ some isomorphism $\alpha_{(X, U)}: R_{\mathrm{tr}}^{\Delta}[Z](1)[1]$ $\stackrel{\sim}{\rightarrow} R_{\mathrm{tr}}^{\Delta}[U / X]$ in $\mathcal{D}_{p \mathcal{M}}^{\text {eff tri }}$ which is identity map for $(X, U)=Z \times \mathbb{T}$ and such that for every correspondence $\theta:\left(X^{\prime}, U^{\prime}\right) \rightarrow(X, U)$ between nice pairs the map $\alpha_{(X, U)}^{-1} \theta^{r e l} \alpha_{\left(X^{\prime}, U^{\prime}\right)}$ : $R_{\mathrm{tr}}^{\Delta}\left[Z^{\prime}\right](1) \rightarrow R_{\mathrm{tr}}^{\Delta}[Z](1)$ equals $S p(\theta)(1)$. Indeed, $\alpha_{(X, U)}$ coincides then with $\theta^{\text {rel }}$ : $R_{\mathrm{tr}}^{\Delta}[Z](1)[1] \rightarrow R_{\mathrm{tr}}^{\Delta}[U / X]$ for every link correspondence $\theta$, hence is canonical.

For a given nice $(X, U)$, choose a nice Voevodsky triple $(X / S, U)$ and an $S$-special $\rho_{(X, U)} \in \operatorname{Cor}(Z \times \mathbb{T},(X, U))$. Set $\alpha_{(X, U)}:=\rho_{(X, U)}^{r e l}$. By (the proof of) (ii) in 4.5.1, 
this is an isomorphism in $\mathcal{D}_{p \mathcal{M}}^{\text {eff tri }}$ whose inverse is the map $\mu_{(X, U)}^{r e l}$ for any $S$-special $\mu_{(X, U)} \in \operatorname{Cor}((X, U), Z \times \mathbb{T})$. Therefore $\alpha_{(X, U)}^{-1} \theta^{r e l} \alpha_{\left(X^{\prime}, U^{\prime}\right)}=\left(\mu_{(X, U)} \theta \rho_{\left(X^{\prime}, U^{\prime}\right)}\right)^{r e l}$. By Remark (ii) in 5.1, one has $S p\left(\mu_{(X, U)} \theta \rho_{\left(X^{\prime}, U^{\prime}\right)}\right)=S p(\theta)$. We are done by 3.4.2 applied to $\mu_{(X, U)} \theta \rho_{\left(X^{\prime}, U^{\prime}\right)} \in \mathcal{C}$ or $\left(Z^{\prime} \times \mathbb{T}, Z \times \mathbb{T}\right)$.

Example. Let $(X, U)$ and $\left(X^{\prime}, U^{\prime}\right)$ be nice pairs and $\pi: X^{\prime} \rightarrow X$ be an étale map such that $\pi^{-1}(Z)=Z^{\prime}$. Consider $\pi$ as a correspondence $\left(X^{\prime}, U^{\prime}\right) \rightarrow(X, U)$; then $S p(\pi)$ equals $\left.\pi\right|_{Z^{\prime}}: Z^{\prime} \rightarrow Z$. By the proposition, for every link correspondences $\theta$, $\theta^{\prime}$ for $(X, U),\left(X^{\prime}, U^{\prime}\right)$ the diagram

$$
\begin{array}{ccc}
R_{\mathrm{tr}}^{\Delta}\left[Z^{\prime}\right](1)[1] & \stackrel{\theta^{\prime r e l}}{\longrightarrow} & R_{\mathrm{tr}}^{\Delta}\left[U^{\prime} / X^{\prime}\right] \\
\left.\pi\right|_{Z^{\prime}}(1) \downarrow & & \pi \downarrow \\
R_{\mathrm{tr}}^{\Delta}[Z](1)[1] & \stackrel{\theta^{r e l}}{\longrightarrow} & R_{\mathrm{tr}}^{\Delta}[U / X]
\end{array}
$$

is homotopically commutative.

5.3. One can use 5.1.1, 5.2 to treat every situation Zariski locally:

Proposition. Let $X$ be a smooth quasi-projective variety, $Z \subset X$ a divisor, $Q \subset X$ a finite set of smooth points. If $k$ is infinite, then $Q$ admits a Zariski neighborhood $V \subset X$ such that $(V, V \backslash Z)$ is a Voevodsky pair. If $Z$ is smooth at $Q \cap Z$, then one can find $V$ such that $(V, V \backslash Z)$ is nice.

Proof. We can assume that $X$ is affine and equidimensional, and (replacing each point in $Q$ by a smooth closed point from its closure) that points of $Q$ are closed.

Consider a closed embedding $X \hookrightarrow K$ where $K$ is a vector space, and compose it with an embedding $K \hookrightarrow L$, where $L$ is another vector space such that every polynomial of degree $\leq 2$ on $K$ is the restriction to $K$ of some linear function on $L$.

Let $L \subset \bar{L}:=\mathbb{P}\left(L \times \mathbb{A}^{1}\right)$ be the open embedding, so $\bar{L} \backslash L=\mathbb{P}(L)$. Let $\bar{X}$ be the closure of $X$ in $\bar{L}$; same for $\bar{Z}$, etc.

Consider a linear projection $\pi: L \rightarrow D$, $\operatorname{dim} D=\operatorname{dim} X-1$. Let $C$ be the closure of $X \subset \bar{L} \times D$, so we have a proper projection $C \rightarrow D$; set $X^{\infty}:=C \backslash X$.

Suppose $\pi$ is generic, i.e., it lies in a sufficiently small open subset in the space of all linear projections (since $k$ is infinite, we can choose such a $\pi$ to be defined over $k$ ). Then $\bar{Z} \backslash Z$ does not intersect the closure of a fiber of $\pi$, hence the map $Z \rightarrow D$ is finite. Similarly, $\bar{X} \backslash X$ intersects it by finitely many points, hence the projection $X^{\infty} \rightarrow D$ is finite. By a Bertini theorem of [SGA4] XI 2.1, ${ }^{35}$ the fibers of $\pi$ passing through points of $Q$ intersect $X$ transversally, and if $Z$ is a divisor smooth at $Q \cap Z$, then these fibers intersect $Z$ transversally at smooth points of $Z$.

Set $\tilde{Q}:=Q \cup\left(Z \cap \pi^{-1} \pi(Q)\right) \subset X$; this is a finite set of points. Consider the line bundle $\mathcal{O}(\Delta)$ on $X \underset{D}{\times} X$. Choose an affine open $W \subset X \underset{D}{\times X}$ which contains $\tilde{Q} \underset{D}{\times} \tilde{Q}$ where $\mathcal{O}(\Delta)$ is trivial. Let $U \subset X$ be an affine open which contains $\tilde{Q}$ and such that $U \underset{S}{\times} \tilde{Q} \subset W$.

\footnotetext{
${ }^{35}$ It is here that we need to replace $K$ by $L$.
} 
Let $S \subset D$ be an affine Zariski neighborhood of $\pi(Q)$; write $X_{S}$, etc., for the pull-back of our schemes to $S$. If $S$ is sufficiently small, then $U_{S}^{\infty}:=C_{S} \backslash U_{S}$ is finite over $S, U_{S}^{\infty} \cap Z_{S}=\emptyset$ and $U_{S}^{\infty} \cup Z_{S}$ admits an affine neighborhood in $C_{S}$; if $Z$ is a smooth at $Q \cap Z$, then $Z_{S}$ is étale over $S$. Our $V / S$ is such an $U_{S}$.

5.4. Proof of the proposition in 4.6.1. By Remark (b) in 4.6.1, we can assume that $k$ is infinite.

(i) Follows from the proposition in 5.3 and (ii) of the proposition in 5.2.

(ii) By 5.3, one can find an open $V \subset U$ which contains the set $\theta(P) \cup \theta^{\prime}(P)$, hence $P$, such that the pair $(V, V \backslash Z)$ is nice. Set $W:=\left\{w \in(V \cap Z) \times \mathbb{A}^{1}\right.$ : $\left.\theta(w) \cup \theta^{\prime}(w) \subset V\right\}$; this is an open neighborhood of $P$ in $(V \cap Z) \times \mathbb{A}^{1}$. For an open affine neighborhood $Z^{\prime}$ of $P$ in $W \cap Z$ set $V^{\prime}:=W \cap\left(Z^{\prime} \times \mathbb{A}^{1}\right)$. If $Z^{\prime}$ is sufficiently small, then $\left(V^{\prime} / Z^{\prime}, V^{\prime} \backslash Z^{\prime}\right)$ is a nice Voevodsky triple, so $\left(V^{\prime}, V^{\prime} \backslash Z^{\prime}\right)$ is a nice pair. Then $\left.\theta\right|_{V^{\prime}},\left.\theta^{\prime}\right|_{V^{\prime}} \in \mathcal{C}$ or $\left(\left(V^{\prime}, V^{\prime} \backslash Z^{\prime}\right),(V, V \backslash Z)\right)$. Since $\operatorname{Sp}\left(\left.\theta\right|_{V^{\prime}}\right)=\operatorname{Sp}\left(\left.\theta^{\prime}\right|_{V^{\prime}}\right)$, the morphisms $\left.\theta^{r e l}\right|_{V^{\prime}},\left.\theta^{\prime r e l}\right|_{V^{\prime}}: R_{\mathrm{tr}}^{\Delta}\left[Z^{\prime}\right](1)[1] \rightarrow R_{\mathrm{tr}}^{\Delta}[(V \backslash Z) / V]$ are homotopic by (iii) of the proposition in 5.2. So their compositions with $R_{\mathrm{tr}}^{\Delta}[(V \backslash Z) / V] \rightarrow R_{\mathrm{tr}}^{\Delta}[(U \backslash Z) / U]$ are homotopic, q.e.d.

5.5. The proof of the theorem in 4.6.3 uses another Bertini-type statement:

Let $L$ be a finite-dimensional vector space. Suppose we have a datum $\left\{\left(Y^{m}, S_{m}\right)\right\}$, $m=1, \ldots, \operatorname{dim} L$, where $Y^{m} \subset L$ is a closed reduced subscheme purely of codimension $m$, and $S_{m} \subset Y^{m}$ is a finite subset of closed smooth points of $Y^{m}$ which meets every irreducible component of $Y^{m}$.

A flag $\Phi=\left(L_{1} \subset L_{2} \subset \ldots\right)$ of linear subspaces of $L$, $\operatorname{dim} L_{m}=m$, is said to be cotransversal to $\left\{\left(Y^{m}, S_{m}\right)\right\}$ if for every $m=1, \ldots, \operatorname{dim} L-1$ the map $Y^{m} \rightarrow L / L_{m}$ is finite and is étale at $S_{m}$, and over a Zariski neighborhood of the image of $S_{m+1}$ in $L / L_{m}$ the projection $Y^{m+1} \rightarrow L / L_{m}$ is an embedding.

Proposition. If $k$ is infinite, then one can find $\Phi$ cotransversal to $\left\{\left(Y^{m}, S_{m}\right)\right\}$.

Proof. We can assume that $\operatorname{dim} L>1$. Let us construct a non-empty Zariski open $U \subset \mathbb{P}(L)$ such that for every line $\ell$ in $U$ all the projections $Y^{m} \rightarrow L / \ell$ are finite, for every $m \geq 2$ the morphism $Y^{m} \rightarrow L / \ell$ is an embedding over a Zariski neighborhood of the image of $S_{m}$, and the projection $Y^{1} \rightarrow L / \ell$ is étale at $S_{1}$.

Consider the open embedding $L \subset \mathbb{P}\left(L \times \mathbb{A}^{1}\right)$, so $\mathbb{P}(L)=\mathbb{P}\left(L \times \mathbb{A}^{1}\right) \backslash L$. Let $\bar{Y}^{m}$ be the closure of $Y^{m}, Y_{\infty}^{m}:=\bar{Y}^{m} \backslash Y^{m} \subset \mathbb{P}(L)$. Let $T^{m} \subset L$ be the union of tangent spaces to $Y^{m}$ at points of $S_{m}$ (considered as affine planes in $L$ ); set $T_{\infty}^{m}:=\bar{T}^{m} \backslash T^{m} \subset \mathbb{P}(L){ }^{36}$ Let $Z^{m} \subset \mathbb{P}(L)$ be the closed subvariety of those points $x$ that for some $s \in S_{m}$ the line passing through $x$ and $s$ intersects $\bar{Y}^{m}$ not only at $s$. Since $\operatorname{dim} Z^{m} \leq \operatorname{dim} Y^{m}$ and $\operatorname{dim} Y_{\infty}^{m} \cup T_{\infty}^{m}<\operatorname{dim} Y^{m}$, the complement to $\left(\cup_{m \geq 2} Z^{m}\right) \cup Y_{\infty}^{1} \cup T_{\infty}^{1}$ in $\mathbb{P}(L)$ is non-empty; this is our $U$.

We construct $\Phi=\left(L_{1} \subset L_{2} \subset \cdots\right)$ by induction by $\operatorname{dim} L$. Take for $L_{1}$ any element of $U(k)$ (which is non-empty since $k$ is infinite). Let $\left(L_{2} / L_{1} \subset L_{3} / L_{1} \subset \ldots\right.$ )

${ }^{36}$ I.e., $T_{\infty}^{m}=\cup_{s \in S_{m}} \mathbb{P}\left(T_{s}\right)$ where $T_{s}$ is the tangent plane to $Y^{m}$ at $s \in S_{m}$ 
be any flag in $L / L_{1}$ cotransversal to the datum of the images of $\left\{\left(Y^{m}, S_{m}\right)\right\}, m \geq 2$, in $L / L_{1}$; one can find it by the induction assumption.

5.6. Proof of the theorem in 4.6.3. As in loc. cit., $k$ is perfect and $F$ is an $\mathbb{A}^{1}$-homotopy invariant presheaf with transfers. By an argument similar to that in Remark (b) in 4.6.1, we can assume that $k$ is infinite.

5.6.1. Let us check that the Cousin differential commutes with the pull-back map $f^{*}$ for a smooth $f: Y \rightarrow X$. The assertion is local, so we can assume that $f$ is the composition of an étale map and a direct product projection; then it suffices to treat either of these types of maps separately. Replacing $X$ by the smooth part of the normalization of its reduced irreducible subscheme of codimension $n$, and $F$ by $F_{-n}$, we see that it suffices to consider $d: \mathcal{C}$ ous $(F)^{0} \rightarrow \operatorname{Cous}(F)^{1}$. We need to check that $f$ commutes with the link correspondences at the generic point of a divisor on $X$ and that of its preimage on $Y$. The étale case follows from (5.2.2). For a direct product projection $Y=P \times X \rightarrow X$ we choose a link correspondence on $Y$ as the direct product of that on $X$ and id ${ }_{P}$; then the assertion is evident.

5.6.2. Let us check that for a proper $g: Z \rightarrow X$ the Cousin differential commutes with the trace map $g_{*}$. Looking at the components $d_{x y}$ (and replacing $F_{-n}$ by $F$ ), we see that the assertion amounts to the next lemma:

Lemma. (i) Let $T, T^{\prime}$ be semi-local schemes as in 4.6.2, and $T^{\prime} \rightarrow T$ be a finite surjective morphism. Then $\operatorname{tr}_{s_{T^{\prime}} / s_{T}} \operatorname{Res}^{T^{\prime}}=\operatorname{Res}^{T} \operatorname{tr}_{\eta_{T^{\prime}} / \eta_{T}}: F\left(\eta_{T^{\prime}}\right) \rightarrow F_{-1}\left(s_{T}\right)$.

(ii) Let $\eta$ be the generic point of a smooth variety, $C / \eta$ a proper irreducible curve, and $Z \subset C$ a reduced subscheme finite over $\eta$ such that $U:=C \backslash Z$ is smooth. Then the composition $F(U) \stackrel{\operatorname{Res}_{Z}}{\longrightarrow} F_{-1}(Z) \stackrel{\operatorname{tr}_{Z / \eta}}{\longrightarrow} F_{-1}(\eta)$ vanishes.

Proof. (i) Passing to the normalizations (as in 4.6.2), we can assume that $T$ and $T^{\prime}$ are regular. One can find a finite morphism $\pi: V^{\prime} \rightarrow V$ of smooth affine varieties and a smooth divisor $Z \subset V$ with $Z^{\prime}:=\pi^{-1}(Z)_{\text {red }}$ smooth, such that $T^{\prime} / T$ is the localization of $V^{\prime} / V$ at the generic point(s) $\eta_{Z}$ of $Z$. Thus $\eta_{Z}=s_{T}, \eta_{Z^{\prime}}=s_{T^{\prime}}$.

Set $\pi_{s}:=\left.\pi\right|_{Z^{\prime}}: Z^{\prime} \rightarrow Z$. The transposed correspondence $\pi^{\vee} \in \mathcal{C}$ or $\left(V, V^{\prime}\right)$ can be seen as a morphism of pairs $(V, V \backslash Z) \rightarrow\left(V^{\prime}, V^{\prime} \backslash Z^{\prime}\right)$; then $S p\left(\pi^{\vee}\right)=\pi_{s}^{\vee}$.

Take any element of $F\left(\eta_{T^{\prime}}\right)$. Shrinking $V$, we can assume that it comes from some $\phi \in F\left(V^{\prime} \backslash Z^{\prime}\right)$. One can find an open $U^{\prime} \subset V^{\prime}$ that contains $\eta_{Z^{\prime}}$ such that the pair $\left(U^{\prime}, U^{\prime} \backslash Z^{\prime}\right)$ is nice, and an open $U \subset V$ with $\eta_{Z} \subset U$ and $\pi^{-1}(U) \subset U^{\prime}$ such that the pair $(U, U \backslash Z)$ is nice (see 5.3). Notice that $\left.\pi^{\vee}\right|_{U} \in \mathcal{C} \operatorname{Cor}\left((U, U \backslash Z),\left(U^{\prime}, U^{\prime} \backslash Z^{\prime}\right)\right)$.

Let $\theta, \theta^{\prime}$ be link correspondences for $(U, U \backslash Z)$ and $\left(U^{\prime}, U^{\prime} \backslash Z^{\prime}\right)$ (see 5.2(ii)). Set $\alpha:=\pi^{\vee} \theta, \beta:=\theta^{\prime}\left(\pi_{s}^{\vee} \times \mathrm{id}_{\mathbb{T}}\right) \in \mathcal{C} \operatorname{or}\left(Z \times \mathbb{T},\left(U^{\prime}, U^{\prime} \backslash Z^{\prime}\right)\right)$. Then $\operatorname{Sp}(\alpha)=$ $\pi_{s}^{\vee}=S p(\beta)$, hence, by 5.2(iii), $\alpha^{\text {rel }}=\beta^{\text {rel }}$. Since $\operatorname{Res}^{T} \operatorname{tr}_{\eta_{T^{\prime}} / \eta_{T}}(\phi)=\alpha^{r e l *}(\phi)$, $t r_{s_{T^{\prime}} / s_{T}} \operatorname{Res}^{T^{\prime}}(\phi)=\beta^{r e l *}(\phi)$, we are done.

(ii) Choose a finite map $C \rightarrow \mathbb{P}_{\eta}^{1}$ which maps $Z$ to $\infty$. Applying (i) to the localizations of $C$ and $\mathbb{P}_{\eta}^{1}$ at $Z$ and $\infty$ respectively, we reduce our statement to the case $C=\mathbb{P}_{\eta}^{1}, Z=\infty$. Here it is evident since $F(U)=F(\eta)$.

5.6.3. Let us check that the square of the Cousin differential vanishes. The 
assertion is local, so we consider an affine variety $X$ and $\phi \in \operatorname{Cous}(F)^{n}(X)$; we want to check that $d_{X}^{2}(\phi)=0$. Let $Y \subset X$ be the support of $\phi$ and $\xi$ be the union of the generic points of the support of $d^{2}(\phi)$.

Let $X \hookrightarrow T$ be any closed embedding in a vector space. By 5.5 , one can find a linear projection $T \rightarrow L$, such that $Y \rightarrow L$ is finite and surjective and $\xi \rightarrow L$ is an embedding. By (i) in the lemma in 5.6.2 applied to $Y / L$ and $F_{-n}$, we see that $d_{X}^{2}(\phi) \in F_{-n-2}(\xi)$ equals $d_{L}^{2} \operatorname{tr}_{Y / L}(\phi)$. So, replacing $F_{-n}$ by $F, X$ by $L$, and $\operatorname{tr}_{Y / L}(\phi)$ by $\phi$, we are reduced to the case $\phi \in \mathcal{C}$ ous $(F)^{0}(L)=F\left(\eta_{L}\right)$.

Let $Y^{1} \subset L$ be a reduced hypersurface such that $\phi$ comes from some $\phi^{\sim} \in$ $F\left(L \backslash Y^{1}\right)$, and $Y^{2} \subset Y^{1}$ be a codimension 1 reduced subvariety such that $Y^{1} \backslash Y^{2}$ is smooth and $d(\phi) \in F_{-1}\left(\eta_{Y^{1}}\right)$ comes from some $d(\phi)^{\sim} \in F_{-1}\left(Y^{1} \backslash Y^{2}\right)$. By 5.5, we can find a decomposition $L=\mathbb{A}^{1} \times D$ such that $Y^{1}$ is finite over $D$ and $\eta_{Y^{2}} \rightarrow D$ is an embedding. By (i) of the lemma in 5.6.2 applied to $Y^{1} / D$ and $d(\phi) \in F_{-1}\left(\eta_{Y^{1}}\right)$, one has $d_{L}^{2}(\phi)=d_{D} \operatorname{tr}_{Y^{1} / D} d_{L}(\phi) \in F_{-2}\left(\eta_{Y^{2}}\right)$.

Consider the open embedding $L=\mathbb{A}^{1} \times D \subset \bar{L}:=\mathbb{P}^{1} \times D$. Then $d_{\bar{L}}(\phi)=d_{L}(\phi)+$ $\operatorname{Res}_{\infty}(\phi)$ where $\operatorname{Res}_{\infty}$ is the residue at $L^{\infty}:=\bar{L} \backslash L=D$. By (ii) of the lemma in 5.6.2 applied to $\bar{L} / D$, one has $\operatorname{tr}_{Y^{1} / D} d_{L}(\phi)=-\operatorname{Res}_{\infty}(\phi) \in F_{-1}\left(\eta_{D}\right)$. Now $\operatorname{Res}_{\infty}(\phi)$ is the restriction to the generic point of $D=L^{\infty}$ of $\operatorname{Res}_{D}\left(\phi^{\sim}\right) \in\left(F_{-1}\right)_{\mathrm{Zar}}(D)$ (see the remark in 4.6.2). Therefore $d_{D} \operatorname{Res}_{\infty}(\phi)=0$, q.e.d.

5.6.4. Let us check that for a smooth semi-local $\hat{X}$ one has $H^{>0} \operatorname{Cous}(F)(\hat{X})$ $=0$. Our $\hat{X}$ is localization of some smooth affine variety $X$ at finitely many points, so $\hat{X}$ is the intersection of semi-local schemes $X_{Q}$, where $Q \subset X$ are some finite collections of closed points. Thus $\operatorname{Cous}(F)(\hat{X})=\underline{\varliminf} \operatorname{Cous}(F)\left(X_{Q}\right)$, and we can assume that $\hat{X}=X_{Q}$.

Let $\phi \in \operatorname{Cous}(F)^{m}\left(X_{Q}\right), m>0$, be any cycle. Since $\operatorname{Cous}(F)\left(X_{Q}\right)$ is the inductive limit of the Cousin complexes of the Zariski neighborhoods of $Q$, we can assume (shrinking $X$ if necessary) that $\phi$ is a cycle in $\operatorname{Cous}(F)(X)$. Let $Y \subset X$ be the support of $\phi$. We look for an open neighborhood $U$ of $Q$ such that $\left.\phi\right|_{U}$ is exact.

Let $X \hookrightarrow T$ be a closed embedding into a vector space. By 5.5 , one can find a linear projection $T \rightarrow L$ such that the projection $\pi: X \rightarrow L$ is finite, surjective, and étale at $Q$, and $\eta_{Y} \rightarrow L$ is an embedding. Consider the cycle $\operatorname{tr}_{X / L}(\phi) \in$ $\operatorname{Cous}(F)^{m}(L)$. Suppose we know that it is exact, i.e., $\operatorname{tr}_{X / L}(\phi)=d_{L}(\psi)$. One can find an open neighborhood $U \subset X$ of $Q$ such that $U / L$ is étale and $\pi^{-1} \pi\left(\eta_{Y}\right) \cap U=$ $\eta_{Y}$. Set $\psi_{U}:=\left(\left.\pi\right|_{U}\right)^{*}(\psi) \in \operatorname{Cous}(F)^{m-1}(U)$. Then $d_{U}\left(\psi_{U}\right)=\left.\phi\right|_{U} ;$ we are done.

It remains to show that $H^{>0} \operatorname{Cous}(F)(L)=0$. We do this by induction by $\operatorname{dim} L$. Write $L=\mathbb{A}^{1} \times D$ and consider the projection $p: L \rightarrow D$. We know that $H^{>0} \operatorname{Cous}(F)(D)=0$ by the induction assumption, so it remains to check that $p^{*}$ : $\operatorname{Cous}(F)(D) \rightarrow \operatorname{Cous}(F)(L)$ is a quasi-isomorphism. Filtering the Cousin complexes as in 4.6.4, we see that $p^{*}$ is, in fact, a filtered quasi-isomorphism (since for any $\xi \in D$ the 2-term complex $\operatorname{Cous}(F)\left(\mathbb{A}_{\xi}^{1}\right)$ is a resolution of $F_{-m}(\xi)=F_{-m}\left(\mathbb{A}_{\xi}^{1}\right)$, say, by 5.1 .2$)$, q.e.d.

5.6.5. It is clear that the image of the map $F(X) \rightarrow \operatorname{Cous}(F)^{0}(X)$ is killed by the Cousin differential. Let us show that for $\hat{X}$ as in 5.6.4, $F(\hat{X}) \stackrel{\sim}{\rightarrow} H^{0} \mathcal{C o u s}(F)(\hat{X})$. 
Our map is injective: this follows from the proposition in 5.3 and (i) of the proposition in 5.1.1. It remains to show that any $\phi_{\eta} \in H^{0} \mathcal{C}$ ous $(F)(\hat{X})$ comes from $F(\hat{X})$. We can assume that $\hat{X}$ is the localization of a smooth affine $X$ at a finite subset $Q \subset X$, and $\phi_{\eta} \in H^{0} \mathcal{C} \operatorname{cous}(F)(X)$. There is a divisor $Z \subset X$ such that $\phi_{\eta}$ comes from some $\phi \in F(X \backslash Z)$. Replace $X$ by $V$ from the proposition in 5.3. Choose a correspondence $s$ from (i) of the proposition in 5.1.1; its action $s^{*}$ is left inverse to the restriction morphism $F(V) \rightarrow F(V \backslash Z)$. So we have $s^{*}(\phi) \in F(V)$. Let us check that $\phi_{\eta}$ equals $s^{*}(\phi)_{\eta}$ (the restriction of $s^{*}(\phi)$ to $\eta=\eta_{X}$ ); this will prove our assertion.

Replacing $\phi$ by $\phi-\left.s^{*}(\phi)\right|_{V \backslash Z}$, we can assume that $s^{*}(\phi)=0$; we want to check that $\phi_{\eta}=0$. Let $\kappa$ be the generic point subscheme of the base of the projection $V \rightarrow S$. Pulling back $(V / S, V \backslash Z)$ to $\kappa$, we get a $\kappa$-curve $V_{\kappa}$, a divisor $Z_{\kappa} \subset V_{\kappa}$, $\phi_{\kappa} \in F\left(V_{\kappa} \backslash Z_{\kappa}\right)$, and, by Remark (i) in 5.1.1, $s_{\kappa} \in \mathcal{C} \operatorname{or}\left(V_{\kappa}, V_{\kappa} \backslash Z_{\kappa}\right)$ such that $s_{\kappa}^{*}\left(\phi_{\kappa}\right)=0$. By (i) of the proposition in 5.1.1 and 5.2, we have a decomposition $F\left(V_{\kappa} \backslash Z_{\kappa}\right) \stackrel{\sim}{\rightarrow} F\left(V_{\kappa}\right) \oplus F_{-1}\left(Z_{\kappa}\right)$ where the first projection is $s_{\kappa}^{*}$ and the second one is $\operatorname{Res}_{Z_{\kappa}}$ (see 4.6.2). Since $\phi$ is killed by the Cousin differential, $\operatorname{Res} Z_{\kappa}\left(\phi_{\kappa}\right)=0$. Thus $\phi_{\kappa}=0$, so $\phi_{\eta}=0$, q.e.d.

\section{$\S$ 6. Playing with motives: first results}

We assume that the base field $k$ is perfect. This section corresponds roughly to the material of lectures 3, 5.9, 12.21-12.28, 13.22-13.27, 16.13-16.25, 20 in [MVW], $3.5,4.1,4.3$ of [Vo2], [Deg1], and $\S 6$ of [Bo1].

6.1. The DG category of motives. Theorem 3.3 remains valid for motives:

Proposition. The Tate motive is homotopically quasi-inversible: on $\mathcal{D}_{\mathcal{M}}^{\mathrm{eff}}{ }^{\mathrm{tri}}$ (or $\underline{\mathcal{D}}_{\mathcal{M}}^{\text {eff }}{ }^{\text {tri }}$ ) the Tate twist endofunctor is fully faithful.

Proof. For $F \in D \mathcal{P S} h_{\mathrm{tr}}$ consider a morphism $\mu_{F}: F \rightarrow\left(\mathcal{C}^{\mathcal{M}}(F(1))\right)_{-1}[-1]$ that comes from the canonical morphism $F(1) \rightarrow \mathcal{C}^{\mathcal{M}}(F(1))$ by adjunction. As in the remark in 3.3, it suffices to check that $\mu_{F}$ is a quasi-isomorphism for $F \in$ $I_{\mathrm{tr}}^{\Delta \perp} \cap I_{\mathrm{tr}}^{\mathrm{Nis} \perp}$. By (4.4.3), this follows from 3.3 and the next lemma:

Lemma. For $G \in I_{\mathrm{tr}}^{\Delta \perp}$ the evident arrow $\mathcal{C}^{\mathrm{Nis}}\left(G_{-1}\right) \rightarrow\left(\mathcal{C}^{\mathrm{Nis}}(G)\right)_{-1}$ is a quasiisomorphism.

Proof of Lemma. We compute the Nisnevich cohomology using the Cousin resolution (see 4.6.3). The pull-back for the projection $\mathbb{G}_{m} \times X \rightarrow X$ yields an embedding $\operatorname{Cous}(G)(X) \hookrightarrow \mathcal{C}$ ous $(G)\left(\mathbb{G}_{m} \times X\right)$. Consider the natural morphism $\operatorname{Cous}\left(G_{-1}\right)(X) \rightarrow \mathcal{C}$ ous $(G)\left(\mathbb{G}_{m} \times X\right) / \mathcal{C}$ ous $(G)(X)$. Both complexes are filtered: the first one by the stupid filtration, the second one by the codimension of the image in $X$ of the support. Our arrow is a filtered quasi-isomorphism by the lemma in 4.6.4 and the theorem in 4.6.3 (cf. 4.6.4), hence is a quasi-isomorphism, q.e.d.

Corollary. For $a, b \geq 0$ there is a canonical homotopy equivalence in $D \mathcal{P} \mathcal{S} h_{\mathrm{tr}}$

$$
\mathcal{C}^{\mathcal{M}}\left(R_{\mathrm{tr}}(a)\right) \stackrel{\sim}{\rightarrow}\left(\mathcal{C}^{\mathcal{M}}\left(R_{\mathrm{tr}}(a+b)\right)\right)_{-b}[-b] .
$$


If $c>a$, then $\left(\mathcal{C}^{\mathcal{M}}\left(R_{\mathrm{tr}}(a)\right)\right)_{-c}=0$. Same is true for $\mathcal{C}^{\mathcal{M}}$ replaced by $\mathcal{C}^{\Delta}$.

Definition. The DG category $\mathcal{D}_{\mathcal{M}}$ of motives is the localization of $\mathcal{D}_{\mathcal{M}}^{\text {eff }}$ by the Tate motive. Explicitly, any motive is represented as $M(a), M \in \mathcal{D}_{\mathcal{M}}^{\text {eff }}, a \in \mathbb{Z}$, and $\operatorname{Hom}_{\mathcal{D}_{\mathcal{M}}}(M(a), N(b)):=\varliminf_{\longrightarrow} \operatorname{Hom}_{\mathcal{D}_{\mathcal{M}}^{\text {eff }}}(M(a+n), N(b+n))$; here the inductive limit is taken for $n \rightarrow+\infty$ and $a+n, b+n$ are assumed to be $\geq 0$.

The homotopy category $\mathcal{D}_{\mathcal{M}}^{\text {tri }}$ is naturally a tensor category: the tensor product is $M(a) \otimes N(b):=(M \otimes N)(a+b)$, and the commutativity constraint is defined using (ii) of the proposition in 3.2.

Remarks. (i) A positive answer to the question in 3.2 would permit us to lift the above tensor structure to a homotopy tensor structure on the DG category $\mathcal{D}_{\mathcal{M}}$.

(ii) The above construction make sense for premotives as well.

6.2. The absolute motivic cohomology. 6.2.1. For $X \in \mathcal{S} m$ and $n \geq 0$ set $^{37}$

$$
R \Gamma_{\mathcal{M}}(X, R(n)):=\operatorname{Hom}_{\mathcal{D}_{\mathcal{M}}^{\text {eff }}}\left(M(X), R_{\mathcal{M}}(n)\right)=\mathcal{C}^{\mathcal{M}}\left(R_{\mathrm{tr}}(n)\right)(X)
$$

and $H_{\mathcal{M}}^{m, n}(X, R):=H^{m} R \Gamma_{\mathcal{M}}(X, R(n))$. So $R \Gamma_{\mathcal{M}}(X, R(*))=R \Gamma\left(X_{\mathrm{Zar}}, R_{\mathrm{tr}}^{\Delta}(*)\right)=$ $R \Gamma\left(X_{\mathrm{Nis}}, R_{\mathrm{tr}}^{\Delta}(*)\right)$ by $4.4,4.5$. For a morphism of smooth varieties $Y \rightarrow X$ we have $R \Gamma_{\mathcal{M}}(Y / X, R(n)):=\mathcal{C}$ one $\left(\mathcal{C}^{\mathcal{M}}\left(R_{\mathrm{tr}}(n)\right)(X) \rightarrow \mathcal{C}^{\mathcal{M}}\left(R_{\mathrm{tr}}(n)\right)(Y)\right)$, etc.

Example. One has $\mathcal{C}^{\mathcal{M}}\left(R_{\mathrm{tr}}(0)\right)=R$, so $R \Gamma_{\mathcal{M}}(X, R(0))=R^{\pi_{0}(X)}$. According to $3.2(\mathrm{i}), 4.4$, one has $\mathcal{C}^{\mathcal{M}}\left(R_{\mathrm{tr}}(1)\right)=\mathcal{O}_{\mathrm{Nis}}^{\times} \stackrel{L}{\otimes} R[-1]$, so

$$
R \Gamma_{\mathcal{M}}(X, R(1))=R \Gamma\left(X_{\mathrm{Zar}}, \mathcal{O}^{\times}\right) \stackrel{L}{\otimes} R[-1]=R \Gamma\left(X_{\mathrm{Nis}}, \mathcal{O}^{\times}\right) \stackrel{L}{\otimes} R[-1] .
$$

As in $3.2, R_{\mathrm{tr}}^{\Delta}(*):=\underset{n \geq 0}{\bigoplus} R_{\mathrm{tr}}^{\Delta}(n)$ is a commutative unital algebra in the ten-

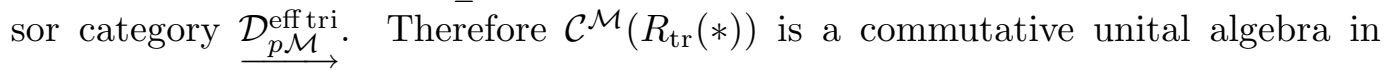
$D \mathcal{P} h_{\text {tr }}$ (see Remark (ii) in 2.3), hence in $D \mathcal{P S h}$ (by the remark in 2.2). Thus $R \Gamma_{\mathcal{M}}(X, R(*))=\oplus R \Gamma_{\mathcal{M}}(X, R(n))$ is a commutative unital $R$-algebra (equipped with an extra grading by $n)$ in the tensor category $D(R)$.

Remark. The positive answer to Question in 3.2 would imply that $R \Gamma_{\mathcal{M}}(X, R(*))$ can be naturally realized as a homotopy object of the category of $E_{\infty}$-algebras.

6.2.2. The motivic cohomology product can also be seen in the next two ways:

(a) The motive $M(X)$ is naturally a cocommutative counital coalgebra in $\mathcal{D}_{\mathcal{M}}^{\text {eff tri. }}$ the coproduct $\delta: M(X) \rightarrow M(X) \otimes M(X)$ comes from the diagonal $X \rightarrow X \times X$, the counit is the augmentation morphism. Now the product of $f_{i} \in H_{\mathcal{M}}^{m_{i}, n_{i}}(X, R)=$ $\operatorname{Hom}_{\mathcal{D}_{\mathcal{M}}^{\text {tri }}}\left(M(X), R_{\mathcal{M}}\left(n_{i}\right)\left[m_{i}\right]\right), i=1,2$, is $f_{1} \cup f_{2}=\pi\left(f_{1} \otimes f_{2}\right) \delta$ where $\pi$ is the product on the algebra $R_{\mathcal{M}}(*):=M\left(R_{\mathrm{tr}}(*)\right)$ in $\mathcal{D}_{\mathcal{M}}^{\text {eff tri }}$.

We see that if $P \in \mathcal{D}_{\mathcal{M}}^{\mathrm{eff}}$ tri is an $M(X)$-comodule, then $P(*)[\cdot]$ is naturally an $H_{\mathcal{M}}^{* *}(X, R)$-module: $\alpha \in H^{m, n}(X, R)$ acts as the composition $P \rightarrow M(X) \otimes P \stackrel{\alpha \otimes \operatorname{id}_{P}}{\longrightarrow}$ $P(n)[m]$. This is the $\cap$-product action.

\footnotetext{
${ }^{37}$ See 2.3 for the notation.
} 
(b) For any $N \in \mathcal{D}_{\mathcal{M}}^{\text {tri }}$ the tensor product $M(X) \otimes N$ is naturally a counital $M(X)$ comodule. For $M(X)$-comodules $P, Q$ in $\mathcal{D}_{\mathcal{M}}^{\text {tri }}$ let $\operatorname{Hom}^{M(X)}(P, Q) \subset \operatorname{Hom}(P, Q)$ be the subgroup of the $M(X)$-comodule morphisms. If $Q=M(X) \otimes N$, then the counit $M(X) \rightarrow R$ defines a projection $\operatorname{Hom}(P, M(X) \otimes N) \rightarrow \operatorname{Hom}(P, N)$ which yields an identification $\operatorname{Hom}^{M(X)}(P, M(X) \otimes N) \stackrel{\sim}{\rightarrow} \operatorname{Hom}(P, N)$. Similarly, for $R_{\mathcal{M}}(*)$ modules $K, L$ we have the subspace $\operatorname{Hom}_{R_{\mathcal{M}}(*)}(K, L) \subset \operatorname{Hom}(K, L)$ of $R_{\mathcal{M}}(*)$ module morphisms; if $K=T(*):=R_{\mathcal{M}}(*) \otimes T$ is an induced $R_{\mathcal{M}}(*)$-module, then the unit $R_{\mathcal{M}} \rightarrow R_{\mathcal{M}}(*)$ defines a projection $\operatorname{Hom}(T(*), L) \rightarrow \operatorname{Hom}(T, L)$ which yields an identification $\operatorname{Hom}_{R_{\mathcal{M}}(*)}(T(*), L) \stackrel{\sim}{\rightarrow} \operatorname{Hom}(T, L)$. Consider now the subalgebra $\operatorname{End}_{R_{\mathcal{M}}(*)}^{M(X)}(M(X)(*)) \subset$ End $(M(X)(*))$ of $M(X)$-comodule and $R_{\mathcal{M}}(*)$-module endomorphisms. So the $\cap$-product action is an isomorphism of graded algebras

$$
H_{\mathcal{M}}^{m, n}(X, R) \stackrel{\sim}{\rightarrow} \operatorname{Hom}_{R_{\mathcal{M}}(*)}^{M(X)}(M(X)(*), M(X)(*+n)[m]) .
$$

For any map $f: Y \rightarrow X$ we have the algebra morphism $f^{*}: H_{\mathcal{M}}^{*}(X, R) \rightarrow$ $H_{\mathcal{M}}^{\cdot *}(Y, R)$, and the morphism $M(Y)(*) \rightarrow M(X)(*)$ is a morphism of $H_{\mathcal{M}}^{*}(X, R)$ modules. Since $M(Y) \rightarrow M(X)$ is a morphism of coalgebras, $M(Y)$ and $M(Y / X):=$ Cone $(M(Y) \rightarrow M(X))[-1]$ are $M(X)$-comodules, hence $M(Y)(*)[\cdot], M(Y / X)(*)[\cdot]$ are $H_{\mathcal{M}}^{*}(X, R)$-modules. As in the previous paragraph, one has a natural isomorphism of $H_{\mathcal{M}}^{* *}(X, R)$-modules

$$
H_{\mathcal{M}}^{m, n}(Y / X, R) \stackrel{\sim}{\rightarrow} \operatorname{Hom}_{R_{\mathcal{M}}(*)}^{M(X)}(M(Y / X)(*), M(X)(*+n)[m]) .
$$

Remarks. (i) For $P, N$ as above the identification $\operatorname{Hom}(P, N) \stackrel{\sim}{\rightarrow} \operatorname{Hom}^{M(X)}(P$, $M(X) \otimes N)$ is $\varphi \mapsto\left(\operatorname{id}_{M(X)} \otimes \varphi\right) \delta_{P}$ where $\delta_{P}$ is the $M(X)$-coaction on $P$. If $P=M(X), N=M(Y)$, then for morphisms given by correspondences it can be described as a map $\operatorname{Cor}(X, Y) \hookrightarrow \operatorname{Cor}(X, X \times Y)$ which assigns to a cycle $c$ on $X \times Y$ its image by $\Delta_{X} \times \operatorname{id}_{Y}: X \times Y \hookrightarrow X \times(X \times Y)$.

(ii) Replacing motives by premotives, we get premotivic cohomology complexes which map naturally to the motivic ones. The above discussion remains valid in this context.

(iii) If $a>0$, then $\operatorname{Hom}(M(X), R(-a))=0$ (see the corollary in 6.1).

6.3. The Gysin equivalence. We refer to [Deg1] for a detailed exposition.

6.3.1. Let $i: Z \hookrightarrow X$ be a closed codimension $n$ embedding of smooth varieties. Set $M(X, X \backslash Z):=M((X \backslash Z) / X)[1]=\mathcal{C}$ one $(M(X \backslash Z) \rightarrow M(X)) \in \mathcal{D}_{\mathcal{M}}^{\text {eff }}$.

Proposition. There is a canonical homotopy equivalence

$$
i^{G y s}: M(X, X \backslash Z) \stackrel{\sim}{\rightarrow} M(Z)(n)[2 n] .
$$

Remarks. (i) Were the question in 4.6 .5 to have a positive answer, this would be immediate. 
(ii) By 4.4, 4.5, the proposition means that for any $\mathbb{A}^{1}$-homotopy invariant complex $F$ of presheaves with transfers there is a natural quasi-isomorphism

$$
i^{G y s *}: R \Gamma\left(Z, F_{-n}\right)[-n] \stackrel{\sim}{\rightarrow} R \Gamma_{Z}(X, F) .
$$

Here the cohomology is that of associated Zariski or Nisnevich sheaves. Notice that if $F$ is a single presheaf, then such a quasi-isomorphism is provided by the morphism $i_{*}$ of the Cousin resolutions (see 4.6.3). One checks easily that the two quasi-isomorphisms coincide (see Exercise (i) below).

Proof. Let $N$ be the normal bundle to $Z$, and $Z \hookrightarrow N$ be the zero section. Now (6.3.1) is the composition of two canonical homotopy equivalences to be defined, respectively, in 6.3 .2 and 6.3 .3 below:

$$
M(X, X \backslash Z) \stackrel{\sim}{\rightarrow} M(N, N \backslash Z) \stackrel{\sim}{\rightarrow} M(Z)(n)[2 n] .
$$

6.3.2. For any scheme $S$ we write $X_{S}$ for $X \times S$ considered as an $S$-scheme; as always, $\mathbb{G}_{m}:=\mathbb{A}^{1} \backslash\{0\} \subset \mathbb{A}^{1}$. Let $Q=Q_{t}$ be the deformation to normal cone for $Z \subset X$ (see e.g. [F] ch. 5). So $Q$ is a smooth $\mathbb{A}^{1}$-scheme together with identifications $\left.Q\right|_{\mathbb{G}_{m}} \stackrel{\sim}{\rightarrow} X_{\mathbb{G}_{m}}, Q_{0} \stackrel{\sim}{\rightarrow} N$, and a closed embedding $Z_{\mathbb{A}^{1}} \hookrightarrow Q$ which are compatible in the evident manner. Thus $Q_{1}=X$, so the embeddings $Q_{1} \hookrightarrow Q \hookleftarrow Q_{0}$ yield morphisms of motives

$$
M(X, X \backslash Z) \rightarrow M\left(Q, Q \backslash Z_{\mathbb{A}^{1}}\right) \leftarrow M(N, N \backslash Z) .
$$

We will see in a moment that these are homotopy equivalences. The first morphism in (6.3.3) is defined then as their composition.

Let us show that the arrows in (6.3.4) yield quasi-isomorphisms between the Hom complexes with values in any motive. This means that for any $\mathbb{A}^{1}$-homotopy invariant complex $F$ of presheaves with transfers the pull-back maps

$$
R \Gamma_{Z}(X, F) \leftarrow R \Gamma_{Z_{\mathbb{A}^{1}}}(Q, F) \rightarrow R \Gamma_{Z}(N, F)
$$

are quasi-isomorphisms; here the cohomology are taken with respect to either the Zariski or the Nisnevich topology (see 4.4, 4.5). The topologies have finite cohomological dimension, so we can assume that $F$ is a single sheaf.

For any closed embedding $i: T \hookrightarrow Y$ of smooth varieties of codimension $n$ one has a canonical identification $i_{*}: R \Gamma\left(Y, F_{-n}\right) \stackrel{\sim}{\rightarrow} R \Gamma_{T}(Y, F)[n]$, see the theorem in 4.6.3 (we represent both complexes by means of the Cousin resolutions). The next general lemma (applied to $Y=Q, T=Z_{\mathbb{A}^{1}}$, and $D=Q_{0}, Q_{1}$ ) together with 4.5 (applied to $F_{-n}$ and $X=Z$ ) imply that the arrows in (6.3.5) are, indeed, quasi-isomorphisms:

Lemma. Let $i: T \hookrightarrow Y$ be as above, $r: D \hookrightarrow Y$ be a smooth divisor transversal to $T$; denote by $i^{\prime}, r^{\prime}$ the embeddings $T \cap D \hookrightarrow D, T \cap D \hookrightarrow T$. Then the diagram

$$
\begin{array}{ccc}
R \Gamma_{T}(Y, F)[n] & \stackrel{r^{*}}{\longrightarrow} & R \Gamma_{T \cap D}(D, F)[n] \\
i_{*} \uparrow & & i_{*}^{\prime} \uparrow \\
R \Gamma\left(T, F_{-n}\right) & \stackrel{r * *}{\longrightarrow} & R \Gamma\left(T \cap D, F_{-n}\right),
\end{array}
$$


where the horizontal arrows are the pull-back maps, commutes.

Proof of Lemma. Since $R i^{!} F_{Y}[n]=H^{n} R i^{!} F_{Y}, R i^{!} F_{D}[n]=H^{n} R i^{\prime !} F_{D}$, (6.3.6) comes by applying the functor $R \Gamma(T, \cdot)$ to the diagram

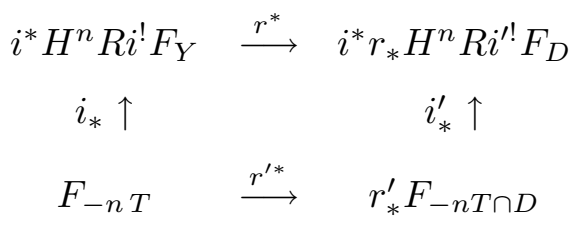

of sheaves on $T$, and we will check the commutativity of (6.3.7). Since $i_{*}^{\prime}$ is an isomorphism and, by (ii) of the theorem in 4.6.3, every local section of $F_{-n T \cap D}$ is uniquely determined by its value at the generic point $\eta_{T \cap D}$, it suffices to check the assertion on a neighborhood of $\eta_{T \cap D}$.

Fit $T$ into a flag of smooth subvarieties $T=T^{n} \stackrel{i_{n}}{\hookrightarrow} \ldots \stackrel{i_{2}}{\hookrightarrow} T^{1} \stackrel{i_{1}}{\hookrightarrow} T^{0}=Y$ such that codim $T^{a}=a$ and $T^{i}$ 's are transversal to $D$. Then $i_{*}: F_{-n T} \rightarrow H^{n} R i^{!} F_{Y}$ can be written as the composition of the !-pull-back to $T$ of the arrows $i_{a *}: F_{-a T^{a}} \stackrel{\sim}{\rightarrow}$

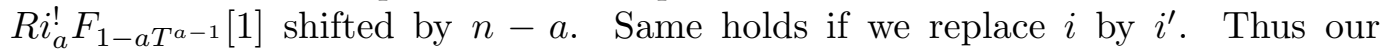
statement for $i$ follows if we prove it for each $i_{a}$ (and $F_{a}$ ). We are reduced to the situation when $T$ is a divisor, i.e., $n=1$.

Shrinking $X$, we can assume that the pair $(X, X \backslash T)$ is nice; let $V$ be a neighborhood of $\eta_{T \cap D}$ in $D$ such that the pair $(V, V \backslash T)$ is also nice. If $\theta, \theta^{\prime}$ are link correspondences for these pairs, then $S p\left(\theta r^{\prime}\right)=i r^{\prime}=r i^{\prime}=S p\left(r \theta^{\prime}\right)$, hence, by 5.2.1(iii), $\theta^{r e l} r^{\prime}=r \theta^{\prime r e l}$. Since $\theta^{r e l}, \theta^{\prime r e l}$ are maps inverse to the vertical arrows in (6.3.7), we are done.

6.3.3. Let us construct the second arrow in (6.3.3). We can forget about $X$, so $N$ is any vector bundle of rank $n$ on a smooth $Z$. Let $Z \hookrightarrow N$ be its zero section, $[Z]$ be the set of connected components of $Z$. First, we define a natural quasi-isomorphism

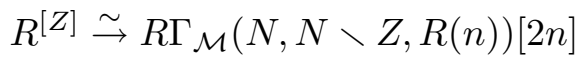

compatible with the base change. It suffices to define (6.3.8) locally with respect to $Z$ (in a way compatible with the base change). So we can assume that $N$ is a trivial vector bundle. Choose a trivialization $\mathbb{A}_{Z}^{n} \stackrel{\sim}{\rightarrow} N$. Consider a covering $\left\{U_{i}\right\}$ of $\mathbb{A}^{n} \backslash\{0\}, U_{i}:=\mathbb{A}^{i-1} \times \mathbb{G}_{m} \times \mathbb{A}^{n-i}$, and its Čech complex $V$. We get identifications $M\left(\mathbb{A}^{n}, \mathbb{A}^{n} \backslash\{0\}\right) \stackrel{\sim}{\leftarrow} M\left(\mathbb{A}^{n}, V.\right)=M\left(\mathbb{A}^{1}, \mathbb{A}^{1} \backslash\{0\}\right)^{\otimes n}=$ $\left(R_{\mathcal{M}}(1)[2]\right)^{\otimes n}=R_{\mathcal{M}}(n)[2 n]$. So the trivialization of $N$ yields an identification $M(N, N \backslash Z) \stackrel{\sim}{\rightarrow} M(Z) \otimes M\left(\mathbb{A}^{n}, \mathbb{A}^{n} \backslash\{0\}\right) \stackrel{\sim}{\rightarrow} M(Z)(n)[2 n]$. We define (6.3.8) as

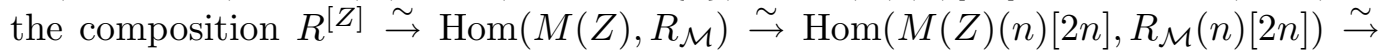
$\operatorname{Hom}\left(M(N, N \backslash Z), R_{\mathcal{M}}(n)[2 n]\right)=R \Gamma_{\mathcal{M}}(N, N \backslash Z, R(n))[2 n]$. Its independence of the trivialization follows since $G L(n)$ is connected.

The generator $1^{[Z]} \in R^{[Z]}$ can be seen, by (6.3.8) and (6.2.4), as a morphism $M(N, N \backslash Z) \stackrel{\sim}{\rightarrow} M((N \backslash Z) / Z)[1] \rightarrow M(Z)(n)[2 n]$ of $M(Z)$-comodules. This is the second arrow in (6.3.3). A moment ago we have checked that it is a homotopy equivalence when $N$ is trivial; the general case reduces to this if we replace $Z$ by a hypercovering $U$. such that $N$ is trivial on $U_{0}$. 
Corollary. Let $F$ be an $\mathbb{A}^{1}$-homotopy invariant complex of presheaves with transfers and $X$ be a smooth variety. Then for the coniveau filtration ${ }^{38}$ on $R \Gamma(X, F)$ one has a canonical quasi-isomorphism $g r^{n} R \Gamma(X, F) \stackrel{\sim}{\rightarrow} \underset{x \in X^{(n)}}{\oplus} F_{-n}\left(\eta_{x}\right)[-n]$.

6.3.4. Exercises. (i) If the above $F$ is a single sheaf, then the filtered complex $R \Gamma(X, F)$ identifies canonically with $\operatorname{Cous}(F)(X)$ equipped with the stupid filtration, and isomorphism (6.3.2) coincides with the identification $i_{*}$ from the theorem in 4.6.3 (we compute both complexes by the Cousin resolution).

(ii) Let $i^{\prime}: Z^{\prime} \hookrightarrow X^{\prime}$ be another embedding as in 6.3.1, and $f: X \rightarrow X^{\prime}$ be a morphism transversal to $Z^{\prime}$ such that $Z=f^{-1}\left(Z^{\prime}\right)$. Then the next diagram commutes:

$$
\begin{array}{ccc}
M(X, X \backslash Z) & \stackrel{f}{\longrightarrow} & M\left(X^{\prime}, X^{\prime} \backslash Z^{\prime}\right) \\
i^{\text {Gys }} \downarrow & & i^{\prime G y s} \downarrow \\
M(Z)(n)[2 n] & \stackrel{\left.f\right|_{Z^{\prime}}}{\longrightarrow} & M\left(Z^{\prime}\right)(n)[2 n] .
\end{array}
$$

E.g., looking at $X^{\prime}=X \times X, Z^{\prime}=X \times Z, f=\Delta$, we see that $i^{\text {Gys }}$ is a morphism of $M(X)$-comodules, hence it commutes with the $\cap$-product action of $H_{\mathcal{M}}^{*}(X, R)$.

(iii) Gysin's identification (6.3.2) for $F=R_{\mathrm{tr}}^{\Delta}(*)$ is an isomorphism

$$
R \Gamma_{\mathcal{M}}(Z, R(*-n))[-2 n] \stackrel{\sim}{\rightarrow} R \Gamma_{\mathcal{M} Z}(X, R(*))
$$

of $R \Gamma_{\mathcal{M}}(X, R(*))$-modules. Let

$$
\operatorname{Res}_{Z}: R \Gamma_{\mathcal{M}}(X \backslash Z, R(*)) \rightarrow R \Gamma_{\mathcal{M}}(Z, R(*-n))[-2 n+1]
$$

be the composition of the inverse to (6.3.10) and the boundary map for $R \Gamma_{\mathcal{M}}$; this is a morphism of $R \Gamma_{\mathcal{M}}(X, R(*))$-modules as well.

(iv) Suppose that $Z$ is a divisor in $X$, so $n=1$, and $R=\mathbb{Z}$. Then (6.2.2) identifies the residue map $\operatorname{Res}_{Z}: H_{\mathcal{M}}^{1,1}(X \backslash Z, \mathbb{Z}(1)) \rightarrow H_{\mathcal{M}}^{0,0}(Z, \mathbb{Z})$ with the map $\operatorname{div}_{Z}: \mathcal{O}^{\times}(X \backslash Z) \rightarrow \mathbb{Z}^{\pi_{0}(Z)}$.

6.4. The class of a cycle. Let $X$ be a smooth variety. Let $Z \subset X$ be a closed subvariety of codimension $\geq n$; denote by $[Z]$ the set of $n$-dimensional irreducible components of $Z$.

Proposition. (i) There is a canonical quasi-isomorphism

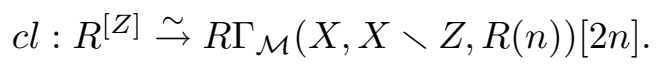

(ii) One has $H_{\mathcal{M}}^{>2 n, n}(X, R(n))=0$. There is a canonical isomorphism

$$
c l: C H^{n}(X) \otimes R \stackrel{\sim}{\rightarrow} H_{\mathcal{M}}^{2 n, n}(X, R) .
$$

\footnotetext{
${ }^{38}$ I.e., the filtration by the codimension of support.
} 
Proof. Consider the coniveau filtration on $T:=R \Gamma_{\mathcal{M}}(X, R(n))$. The corollaries in 6.3 and 6.1 imply that $\operatorname{gr}^{>n} T=0$ and $\operatorname{gr}^{n} T[2 n]=\mathcal{Z}^{n}(X) \otimes R$ (the group of codimension $n$ cycles on $X$ ). Since $\operatorname{gr}^{<n} T$ is the same for $X$ and $X \backslash Z$, we get (i). Since $H^{>m} \mathcal{C}^{\Delta}(R(m))=0$, one has $H^{\geq 2 n} \mathrm{gr}^{<n} T=H^{2 n-1} \mathrm{gr}^{<n-1} T=0$. Therefore $H_{\mathcal{M}}^{2 n, n}(X, R)$ equals the cokernel of the differential $H^{2 n-1} \mathrm{gr}^{n-1} T \rightarrow H^{2 n} \mathrm{gr}^{n} T=$ $\mathcal{Z}^{n}(X) \otimes R$.

Now $H^{2 n-1} \mathrm{gr}^{n-1} T=\underset{x \in X^{(n-1)}}{\oplus} \mathcal{O}^{\times}\left(\eta_{x}\right) \otimes R$ by (6.2.2). By Exercise (iv) in 6.3.4, the above differential sends a rational function $\phi \in \mathcal{O}^{\times}\left(\eta_{x}\right)$ to its divisor $\operatorname{div}(\phi) \in$ $\mathcal{Z}^{n}(X)$. Hence the cokernel equals $C H^{n}(X) \otimes R$, q.e.d.

Exercise. Suppose that $Z$ is smooth irreducible of codimension $n$. Then $\operatorname{cl}(Z) \in$ $H_{\mathcal{M}}^{2 n, n}(X, X \backslash Z, R)$ equals the composition $M(X, X \backslash Z) \stackrel{\sim}{\rightarrow} M(Z)(n)[2 n] \rightarrow$ $R(n)[2 n]$, where the first arrow is the Gysin map, the second one is the augmentation for $M(Z)$. Thus the composition $M(X, X \backslash Z) \stackrel{\sim}{\rightarrow} M(Z)(n)[2 n] \rightarrow M(X)(n)[2 n]$ is the morphism of $M(X)$-comodules that corresponds to $\operatorname{cl}(Z)$ (see 6.2.2). The $\cap$-product action $M(X) \rightarrow M(X)(n)[2 n]$ of $c l(Z)$ (see (6.2.3)) equals the composition $M(X) \rightarrow M(X, X \backslash Z) \stackrel{\sim}{\rightarrow} M(Z)(n)[2 n] \rightarrow M(X)(n)[2 n]$, the middle arrow is the Gysin map, the other two are evident morphisms.

Remark. According to [MVW] 19.1, for every $m, n$ there is a natural isomorphism $H_{\mathcal{M}}^{m, n}(X, \mathbb{Z}) \stackrel{\sim}{\rightarrow} C H^{n}(X, 2 n-m)$ where the r.h.s. is Bloch's higher Chow group.

6.5. The motive of a projective bundle. For a line bundle $\mathcal{L}$ on $X$ denote by $c_{1}^{\mathcal{M}}(\mathcal{L})$ its class in $\operatorname{Pic}(X) \otimes R \cong H_{\mathcal{M}}^{2,1}(X, R)$, see $(6.2 .2)$.

Exercise. If $\lambda$ is a non-zero rational section of $\mathcal{L}$, then $c_{1}^{\mathcal{M}}(\mathcal{L})=\operatorname{cl}(\operatorname{div}(\lambda))$.

Let now $\mathcal{E}$ be any vector bundle of rank $n+1$ on $X$, and $p: \mathbb{P}(\mathcal{E}) \rightarrow X$ be the corresponding projective bundle. Set $c:=c_{1}^{\mathcal{M}}(\mathcal{O}(1)) \in H_{\mathcal{M}}^{2,1}(\mathbb{P}(\mathcal{E}), R)$. As in (6.2.3), let us consider $c^{a} \in H_{\mathcal{M}}^{2 a, a}(\mathbb{P}(\mathcal{E}), R)$ as morphisms $M(\mathbb{P}(\mathcal{E})) \rightarrow M(\mathbb{P}(\mathcal{E}))(a)[2 a]$. Composing with $p$, we get the morphisms $p_{*} c^{a}: M(\mathbb{P}(\mathcal{E})) \rightarrow M(X)(a)[2 a]$.

Proposition. One has a natural homotopy equivalence

$$
\left(p_{*} c^{a}\right): M(\mathbb{P}(\mathcal{E})) \stackrel{\sim}{\rightarrow} \underset{0 \leq a \leq n}{\oplus} M(X)(a)[2 a]
$$

Proof. Choose a covering $\left\{U_{\alpha}\right\}$ of $X$ such that $\mathcal{E}$ is trivial on $U_{\alpha}$ 's. Let $U$. be the corresponding Čech hypercovering, so $M(U.) \stackrel{\sim}{\rightarrow} M(X), M\left(\mathbb{P}(\mathcal{E})_{U}\right) \stackrel{\sim}{\rightarrow} M(\mathbb{P}(\mathcal{E}))$. Consider the corresponding filtrations on $M(X), M(\mathbb{P}(\mathcal{E}))$. The operators $c^{i}$ and $p_{*}$ act naturally on the filtered objects. To show that (6.5.1) is a homotopy equivalence, it suffices to check that such is the associated graded map, which is $\left(p_{*} c^{a}\right): M\left(\mathbb{P}(\mathcal{E})_{U_{m}}\right) \rightarrow \oplus M\left(U_{m}\right)(a)[2 a]$. Thus we can assume that $\mathcal{E}$ is a trivial vector bundle, so $\mathbb{P}(\mathcal{E})=\mathbb{P}_{X}^{n}$.

Consider the embeddings $\mathbb{P}_{X}^{n-1} \hookrightarrow \mathbb{P}_{X}^{n}$ and $\mathbb{A}_{X}^{n}:=\mathbb{P}_{X}^{n} \backslash \mathbb{P}_{X}^{n-1} \stackrel{j}{\hookrightarrow} \mathbb{P}_{X}^{n}$. Since $c=\operatorname{cl}\left(\mathbb{P}_{X}^{n-1}\right)$, the exercise in 6.5 implies that $p_{*} c^{a}$ for $a \geq 1$ equals the composition $M\left(\mathbb{P}_{X}^{n}\right) \stackrel{\alpha}{\rightarrow} M\left(\mathbb{P}_{X}^{n}, \mathbb{A}_{X}^{n}\right) \stackrel{\sim}{\rightarrow} M\left(\mathbb{P}_{X}^{n-1}\right)(1)[2] \stackrel{c^{a-1}}{\longrightarrow} M\left(\mathbb{P}_{X}^{n-1}\right)(a)[2 a] \rightarrow M(X)(a)[2 a]$ 
where the right arrow comes from the projection $\mathbb{P}_{X}^{n-1} \rightarrow X$. Since the composition $M\left(\mathbb{A}_{X}^{n}\right) \stackrel{j_{*}}{\rightarrow} M\left(\mathbb{P}_{X}^{n}\right) \stackrel{p_{*}}{\rightarrow} M(X)$ is a homotopy equivalence, we see that the morphism $M\left(\mathbb{P}_{X}^{n}\right) \stackrel{\left(\alpha, p_{*}\right)}{\longrightarrow} M\left(\mathbb{P}_{X}^{n}, \mathbb{A}^{n}\right) \oplus M(X)$ is also a homotopy equivalence. Together with the previous assertion, this proves (6.5.1) by induction by $n$.

We see that there is a canonical quasi-isomorphism

$$
\underset{0 \leq a \leq n}{\oplus} R \Gamma_{\mathcal{M}}(X, R(m-a))[-2 a] \stackrel{\sim}{\rightarrow} R \Gamma_{\mathcal{M}}(\mathbb{P}(\mathcal{E}), R(m))
$$

which sends $\left(\phi_{a}\right)$ to $\sum \phi_{a} c^{a}$. So one can define the Chern $\operatorname{classes} c_{i}^{\mathcal{M}}(\mathcal{E}) \in H_{\mathcal{M}}^{2 i, i}(X, R)$ by the usual Grothendieck procedure.

6.6. The motive of a blow-up. Let $i: Z \hookrightarrow X$ be a closed embedding of smooth varieties of codimension $m$ and $f: \tilde{X} \rightarrow X$ be the blow-up. Set $\tilde{Z}:=f^{-1}(Z), U:=\tilde{X} \backslash \tilde{Z}=X \backslash Z$; then $\tilde{Z}=\mathbb{P}(N)$ where $N$ is the normal bundle to $Z$ in $X$. Let $\alpha$ be the composition $M(\tilde{X}) \rightarrow M(\tilde{X}, U) \stackrel{\sim}{\rightarrow}$ $M(\mathbb{P}(N))(1)[2] \stackrel{\sim}{\rightarrow} \underset{0<a \leq m}{\oplus} M(Z)(a)[2 a] \rightarrow \underset{0<a<m}{\oplus} M(Z)(a)[2 a]$, where the second morphism is the Gysin equivalence (6.3.1), the third one is (6.5.1), the last arrow is the projection.

Proposition. The map $(f, \alpha)$ is a quasi-isomorphism:

$$
M(\tilde{X}) \stackrel{\sim}{\rightarrow} M(X) \oplus \underset{0<a<m}{\oplus} M(Z)(a)[2 a] .
$$

Proof. $(f, \alpha)$ is compatible with the evident morphisms from $M(U)$ to the l.h.s. and r.h.s. of (6.6.1). So it suffices to show that the corresponding morphism of cones $M(\tilde{X}, U) \stackrel{\stackrel{\sim}{\rightarrow}}{\rightarrow} M(X, U) \oplus \underset{0<a<m}{\oplus} M(Z)(a)[2 a]$ is a quasi-isomorphism.

By the construction of $\alpha$, the assertion follows if we check that the composition $\xi_{1}$ of $M(\tilde{X}, U) \stackrel{\sim}{\rightarrow} M(\mathbb{P}(N))(1)[2] \stackrel{\sim}{\rightarrow} \underset{0<a \leq m}{\oplus} M(Z)(a)[2 a] \rightarrow M(Z)(m)[2 m]$ equals the composition $\xi_{2}$ of $M(\tilde{X}, U) \stackrel{f}{\rightarrow} M(X, U) \stackrel{i^{G y s}}{\longrightarrow} M(Z)(m)[2 m]$.

Looking at the first step of the construction of the Gysin equivalence (the first isomorphism in (6.3.3)) described in 6.3.2, we see that it is compatible with the above constructions. So we can assume that $X$ is a vector bundle $N$ over $Z, i$ is its zero section, $\tilde{X}$ is the space $L$ of the line bundle $\mathcal{O}(-1)$ over $\mathbb{P}(N), f$ is the corresponding standard map. One has a commutative diagram

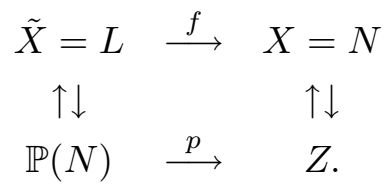

We are playing with $Z$-schemes, so our motives are $M(Z)$-comodules (see 6.2.2). By the exercise in 6.4 and $6.5, \xi_{1}$ is the morphism of $M(Z)$-comodules that corresponds to $\operatorname{cl}(\tilde{Z}) \cup c_{1}(\mathcal{O}(1))^{\cup n-1} \in H_{\mathcal{M}}^{2 n, n}(\tilde{X}, U ; R)$, and $\xi_{2}$ to $f^{*} c l(Z)$. It remains 
to check that these classes are equal. As in 6.3.3, one has $H_{\mathcal{M}}^{2 n, n}(\tilde{X}, U ; R)=R^{[Z]}$, so we can assume that $Z=\operatorname{Spec} k$. The rest is left to the reader.

Remark. The proposition implies that after the motivic localization the complex

$$
R_{\mathrm{tr}}[\tilde{Z}] \rightarrow R_{\mathrm{tr}}[\tilde{X}] \oplus R_{\mathrm{tr}}[Z] \rightarrow R_{\mathrm{tr}}[X]
$$

becomes quasi-isomorphic to 0 . We will see in 6.9.3 that, if resolution of singularities is available, this statement is valid without smoothness assumption on $X$ and $Z$.

6.7. Poincaré duality. 6.7.1. We live in a tensor category with unit object 1. As in [D2], a duality datum consists of a pair of objects $V, V^{*}$ and morphisms $\epsilon: V^{*} \otimes V \rightarrow \mathbf{1}, \delta: \mathbf{1} \rightarrow V \otimes V^{*}$ such that both compositions $V \stackrel{\delta \otimes \text { id }_{V}}{\longrightarrow} V \otimes V^{*} \otimes$ $V \stackrel{\mathrm{id}_{V} \otimes \epsilon}{\longrightarrow} V$ and $V^{*} \stackrel{\operatorname{id}_{V^{*}} \otimes \delta}{\longrightarrow} V^{*} \otimes V \otimes V^{*} \stackrel{\epsilon \otimes \mathrm{id}_{V^{*}}}{\longrightarrow} V^{*}$ are the identity morphisms. For given $V$ the triple $\left(V^{*}, \epsilon, \delta\right)$ is uniquely defined (if it exists), and called the dual object to $V$. Since $\delta$ is determined uniquely by $\epsilon$ (and vise versa), it suffices to specify either of them.

Now our tensor category is $\mathcal{D}_{\mathcal{M}}^{\text {tri }}$. For a smooth variety $X$ of dimension $n$ let

$$
\epsilon_{X}^{\prime}: M(X) \otimes M(X) \rightarrow R_{\mathcal{M}}(n)[2 n]
$$

be the composition $M(X) \otimes M(X) \stackrel{\sim}{\rightarrow} M(X \times X) \stackrel{c l(\Delta)}{\longrightarrow} R_{\mathcal{M}}(n)[2 n]$ where $\operatorname{cl}(\Delta) \in$ $H_{\mathcal{M}}^{2 n, n}(X \times X, R)$ is the class of the diagonal cycle $\Delta: X \hookrightarrow X \times X$. Equivalently, this is the composition $M(X \times X) \rightarrow M(X \times X,(X \times X) \backslash \Delta(X)) \stackrel{\sim}{\rightarrow} M(X)(n)[2 n] \rightarrow$ $R_{\mathcal{M}}(n)[2 n]$; here $\stackrel{\sim}{\rightarrow}$ is the Gysin map, the last arrow is the augmentation. Set $\epsilon_{X}:=\epsilon_{X}^{\prime}(-n)[-2 n]: M(X)(-n)[-2 n] \otimes M(X) \rightarrow R_{\mathcal{M}}$.

Proposition. If $X$ is proper, then $\left(M(X)(-n)[-2 n], \epsilon_{X}\right)$ is the dual to $M(X)$.

Proof. We can assume that $X$ is irreducible and, by an argument similar to one in Remark (b) in 4.6.1, that $k$ is infinite.

Let $\delta_{X}$ be the composition $R_{\mathcal{M}}(n)[2 n] \stackrel{\kappa}{\rightarrow} M\left(\mathbb{P}^{n}\right) \stackrel{\nu}{\rightarrow} M(X) \stackrel{\Delta}{\rightarrow} M(X \times X)$, where $\kappa$ is a component of $(6.5 .1)$, and $\nu \in \mathcal{C}$ or $\left(\mathbb{P}^{n}, X\right)$ is any correspondence whose graph is an irreducible cycle $\Gamma_{\nu} \subset X \times \mathbb{P}^{n}$ such that at the generic point of $\Gamma_{\nu}$ the projection $\Gamma_{\nu} \rightarrow X$ is an isomorphism and the one $\Gamma_{\nu} \rightarrow \mathbb{P}^{n}$ is étale ( $\nu$ exists by Chow's lemma [EGA II] 5.6.1, and 5.5). Set $\delta_{X}:=\delta_{X}^{\prime}(-n)[-2 n]: R_{\mathcal{M}} \rightarrow$ $M(X) \otimes M(X)(-n)[-2 n]$.

Let us check that $\left(\epsilon_{X}, \delta_{X}\right)$ is a duality datum. Our $\epsilon_{X}^{\prime}$ and $\delta_{X}^{\prime}$ are symmetric, so we need to show that the composition

$$
M(X)(n)[2 n] \stackrel{\beta}{\longrightarrow} M(X \times X \times X) \stackrel{\alpha}{\longrightarrow} M(X)(n)[2 n]
$$

where $\alpha:=\operatorname{id}_{M(X)} \otimes \epsilon_{X}^{\prime}$ and $\beta:=\delta_{X}^{\prime} \otimes \operatorname{id}_{M(X)}$, is the identity morphism.

(a) Let $p_{a}: X \times X \rightarrow X, a=1,2$, and $p r_{i}, p r_{i j}: X \times X \times X \rightarrow X, X \times X$, $i, j=1,2,3$ be the projections. Then $\alpha$ equals the $\cap$-product action of $\operatorname{pr}_{23}^{*}(\operatorname{cl}(\Delta))$ 
followed by $p r_{1}$. The pull-back of $p r_{23}^{*}(\operatorname{cl}(\Delta))$ by $\Delta \times \operatorname{id}_{X}: X \times X \rightarrow X \times X \times X$ equals $c l(\Delta)$, so the composition $\gamma$ of

$$
M(X \times X) \stackrel{\Delta \times \mathrm{id}_{X}}{\longrightarrow} M(X \times X \times X) \stackrel{\alpha}{\longrightarrow} M(X)(n)[2 n]
$$

equals $\tilde{c l}(\Delta):=$ the $\cap$-product action of $c l(\Delta)$, followed by $p_{1}$.

Notice that $\tilde{c l}(\Delta)$ equals the composition

$M(X \times X) \rightarrow M(X \times X,(X \times X) \backslash \Delta(X)) \stackrel{\sim}{\rightarrow} M(X)(n)[2 n] \stackrel{\Delta}{\longrightarrow} M(X \times X)(n)[2 n]$

(see Exercise in 6.4). The transposition $\sigma$ of factors of $X \times X$ acts on our objects; since it acts trivially on the term $M(X)(n)[2 n]$, we see that $\tilde{c l}(\Delta)$ is invariant with respect to the left and right compositions with the transposition. Therefore $\gamma=p_{1} \sigma \tilde{c l}(\Delta)=p_{2} \tilde{c l}(\Delta)$.

Now $p_{2}$ defines on $M(X \times X)$ an $M(X)$-comodule structure (see 6.2.2). Our $\gamma$ is a morphism of $M(X)$-comodules which corresponds to $c l(\Delta)$ via the identification $\operatorname{Hom}^{M(X)}(M(X \times X), M(X)(n)[2 n]) \stackrel{\sim}{\rightarrow} \operatorname{Hom}\left(M(X \times X), R_{\mathcal{M}}(n)[2 n]\right)=H_{\mathcal{M}}^{2 n, n}(X \times$ $X, R)$ (see $6.2 .2(\mathrm{~b})$; here $\stackrel{\sim}{\rightarrow}$ is the composition with the augmentation).

(b) Set $\nu_{X}:=\nu \otimes \operatorname{id}_{M(X)}: M\left(\mathbb{P}^{n} \times X\right) \rightarrow M(X \times X)$; this is a morphism of $M(X)$ comodules. By (a), the composition $M\left(\mathbb{P}^{n} \times X\right) \stackrel{\nu_{X}}{\rightarrow} M(X \times X) \stackrel{\gamma}{\rightarrow} M(X)(n)[2 n]$ is a morphism of $M(X)$-comodules that corresponds to $\nu_{X}^{*}(c l(\Delta))$ via the identification $\operatorname{Hom}^{M(X)}\left(M\left(\mathbb{P}^{n} \times X\right), M(X)(n)[2 n]\right) \stackrel{\sim}{\rightarrow} \operatorname{Hom}\left(M\left(\mathbb{P}^{n} \times X\right), R_{\mathcal{M}}(n)[2 n]\right)=$ $H_{\mathcal{M}}^{2 n, n}\left(\mathbb{P}^{n} \times X, R\right)$. By $(6.3 .9)$, the conditions on $\nu$ imply that $\nu_{X}^{*}(\operatorname{cl}(\Delta))=\operatorname{cl}\left(\Gamma_{\nu}\right)$.

(c) Set $j_{X}:=j \otimes \operatorname{id}_{M(X)}: M(X)(n)[2 n] \rightarrow M\left(\mathbb{P}^{n} \times X\right)$; this is a morphism of $M(X)$-comodules. One has $\alpha \beta=\gamma \nu_{X} j_{X}: M(X)(n)[2 n] \rightarrow M(X)(n)[2 n]$. By (b), this is the $M(X)$-comodule endomorphism that corresponds to the class $j_{X}^{*}\left(c l\left(\Gamma_{\nu}\right)\right) \in \operatorname{Hom}\left(M(X)(n)[2 n], R_{\mathcal{M}}(n)[2 n]\right)=H_{\mathcal{M}}^{0}(X, R(0))=R$.

Consider the Künneth decomposition for the Chow groups $H_{\mathcal{M}}^{2 n, n}\left(\mathbb{P}^{n} \times X, R\right)=$ $\sum H_{\mathcal{M}}^{2(n-a), n-a}\left(\mathbb{P}^{n}, R\right) \otimes H_{\mathcal{M}}^{2 a, a}(X, R)=\sum H_{\mathcal{M}}^{2 a, a}(X, R)$ (see (6.5.2)). It shows that for a cycle $Z$ the number $j_{X}^{*}(\operatorname{cl}(Z))$ is simply the degree of $Z$ over the generic point of $X$. Thus $j_{X}^{*}\left(c l\left(\Gamma_{\nu}\right)\right)=1$, so $\alpha \beta$ is the identity morphism, q.e.d.

6.7.2. If a tensor category is Karoubian, then its objects that admit a dual form a Karoubian subcategory. So for a Karoubian tensor triangulated category its objects that admit a dual form a thick subcategory. If resolution of singularities is available, then $\mathcal{D}_{\mathcal{M}}^{\text {tri }}$ is the idempotent completion of the triangulated subcategory strongly generated by motives of proper smooth varieties, hence

Corollary. If $k$ admits resolution of singularities, then $\mathcal{D}_{\mathcal{M}}^{\text {tri }}$ is a rigid tensor category, i.e., each of its objects admits a dual.

See 6.9.4 for an explicit construction of the dual to $M(X)$ for non-compact $X$.

6.7.3. If $P, Q$ are objects of a tensor category such that $P$ admits dual $P^{*}$, then $\operatorname{Hom}(Q, P)=\operatorname{Hom}\left(Q \otimes P^{*}, \mathbf{1}\right)$. This is formula (6.7.3) below; the rest of the corollary is an exercise for the reader: 
Corollary. For X, Y smooth, X proper, one has a natural identification

$$
\operatorname{Hom}(M(Y), M(X)) \stackrel{\sim}{\rightarrow} R \Gamma_{\mathcal{M}}(X \times Y, R(\operatorname{dim} X))[2 \operatorname{dim} X]
$$

Thus $H^{0} \operatorname{Hom}(M(Y), M(X))=C H^{\operatorname{dim} X}(X \times Y) \otimes R$ and $H^{>0} \operatorname{Hom}(M(Y), M(X))$ $=0$. For $c \in \mathcal{C}$ or $(X, Y)$ the class in $C H^{\operatorname{dim} X}(X \times Y)$ of $M(Y) \stackrel{c}{\rightarrow} M(X)$ equals the class of $c$ as of a cycle on $X \times Y$. The composition of morphisms of motives of proper varieties equals the composition of Chow correspondences.

6.7.4. Consider an additive $R$-category of smooth projective varieties and Chow correspondences: $\operatorname{Hom}(Y, X)=C H^{\operatorname{dim} X}(X \times Y) \otimes R$. Its idempotent completion is the category $\mathcal{C} \mathcal{H}_{\mathcal{M}}^{\text {eff }}$ of effective Chow motives. Let $\mathcal{C} \mathcal{H}_{\mathcal{M}}^{\text {eff pretr }}$ be the DG tensor category of finite complexes in $\mathcal{C} \mathcal{H}_{\mathcal{M}}^{\text {eff }}$, and $\mathcal{C} \mathcal{H}_{\mathcal{M}}^{\text {eff tri }}$ be its homotopy category. The next result is due to M. Bondarko [Bo1] $\S 6$ :

Proposition. For $k$ that admits resolution of singularities, there is a natural homotopy tensor DG functor

$$
\epsilon: \mathcal{D}_{\mathcal{M}}^{\text {eff }} \rightarrow \mathcal{C} \mathcal{H}_{\mathcal{M}}^{\text {eff pretr }}
$$

The functor $\epsilon^{\text {tri }}: \mathcal{D}_{\mathcal{M}}^{\text {eff tri }} \rightarrow \mathcal{C} \mathcal{H}_{\mathcal{M}}^{\text {eff tri }}$ is conservative and yields an isomorphism of the $K_{0}$-groups.

Proof. Let $\mathcal{B}$ be the full DG subcategory in $\mathcal{D}_{\mathcal{M}}^{\text {eff }}$ formed by those motives that are isomorphic in $\mathcal{D}_{\mathcal{M}}^{\text {eff tri }}$ to direct summands of motives of smooth proper varieties. By 6.7.3, $B:=\operatorname{Hot} \mathcal{B}$ equals $\mathcal{C} \mathcal{H}_{\mathcal{M}}^{\mathrm{eff}}$, and for every $M, N \in \mathcal{B}$ and $n>0$ one has $H^{>0} \operatorname{Hom}(M, N)=0$. By (iii) of the proposition in 1.5.6, the triangulated subcategory of $\mathcal{D}_{\mathcal{M}}^{\text {eff tri }}$ strongly generated by $B$ is Karoubian. Therefore, by the assumption on $k$, it equals $\mathcal{D}_{\mathcal{M}}^{\text {eff tri }}$. Now we can apply 1.5 .6 to $\mathcal{A}=\mathcal{D}_{\mathcal{M}}^{\text {eff }}$ and $B$. The compatibility of $\epsilon$ with the tensor products is evident.

6.8. The Steinberg relation. Below $R=\mathbb{Z}$. Let $X$ be a smooth variety, $f, g \in \mathcal{O}^{\times}(X)=H_{\mathcal{M}}^{1,1}(X, \mathbb{Z})$ be invertible functions; we get $f \cup g \in H_{\mathcal{M}}^{2,2}(X, \mathbb{Z})$.

Proposition. If $f+g=1$, then $f \cup g=0$.

Proof. An observation: Suppose $X$ is the complement to a smooth divisor $D$ in a smooth variety $\bar{X}$. We have the map $\operatorname{Res}_{D}: H_{\mathcal{M}}^{\cdot}(X, \mathbb{Z}) \rightarrow R \Gamma_{\mathcal{M}}^{\cdot-1,-1}(D, \mathbb{Z})$ (see (6.3.11)) which is a morphism of $H_{\mathcal{M}}(\bar{X}, \mathbb{Z})$-modules. By Exercise (iv) in 6.3.4, for $g \in H_{\mathcal{M}}^{1,1}(X, \mathbb{Z})=\mathcal{O}^{\times}(X)$ one has $\operatorname{Res}_{D}(g)=\operatorname{div}_{D}(g)$. Therefore if $f \in \mathcal{O}^{\times}(\bar{X})$, then $\operatorname{Res}_{D}(f \cup g) \in H_{\mathcal{M}}^{1,1}(D, \mathbb{Z})=\mathcal{O}^{\times}(D)$ equals $\left(\left.f\right|_{D}\right)^{d i v_{D}(g)}$ (see Exercise below for a general statement). In particular, if $f$ and $g$ are such that on each irreducible component of $D$ either $f$ or $g$ is regular and equals 1 , then $\operatorname{Res}_{D}(f \cup g)$ vanishes.

To prove the proposition, it suffices to consider the universal situation of $X=$ $\mathbb{A}^{1} \backslash D$, where $D=\{0,1\}, f=t, g=1-t$. Set $\alpha:=f \cup g$. By the observation, $\operatorname{Res}_{D}(\alpha)=0$, so the exact sequence $0 \rightarrow H_{\mathcal{M}}^{2,2}\left(\mathbb{A}^{1}, \mathbb{Z}\right) \rightarrow H_{\mathcal{M}}^{2,2}(X, \mathbb{Z}) \stackrel{\operatorname{Res}_{D}}{\longrightarrow} k^{\times} \times k^{\times} \rightarrow$ 0 implies that $\alpha \in H_{\mathcal{M}}^{2,2}\left(\mathbb{A}^{1}, \mathbb{Z}\right)=H_{\mathcal{M}}^{2,2}(k, \mathbb{Z})$. Since $\cup$ is skew-commutative, the symmetry $t \mapsto 1-t$ of $X$ sends $\alpha$ to $-\alpha$. Since symmetries of $X$ act on the 
subgroup $H_{\mathcal{M}}^{2,2}(k, \mathbb{Z})$ of $H_{\mathcal{M}}^{2,2}(X, \mathbb{Z})$ as identity, one has $2 \alpha=0$. Thus $t^{2} \cup(1-t)=$ $0=t^{2} \cup(1+t)$, so the element $t^{2} \cup\left(1-t^{2}\right)=t^{2} \cup(1-t)+t^{2} \cup(1+t)$ of $H_{\mathcal{M}}^{2,2}\left(\mathbb{A}^{1} \backslash\{0, \pm 1\}, \mathbb{Z}\right)$ vanishes. But this is the pull-back of $\alpha$ by the map $t \mapsto t^{2}$. The latter map acts as identity on the subgroup $H_{\mathcal{M}}^{2,2}(k, \mathbb{Z})$, so $\alpha=0$, q.e.d.

Exercise. In the situation of the above observation, for any $f, g \in \mathcal{O}^{\times}(X)$ the function $\operatorname{Res}_{D}(f \cup g) \in \mathcal{O}^{\times}(X)$ coincides with the tame symbol $\{f, g\}_{D}$.

Recall that if $X$ is a local scheme, then the Milnor ring $K^{M}(X)$ is a graded associative ring generated by $K_{1}^{M}(X):=\mathcal{O}^{\times}(X)$ modulo the Steinberg relation $\{f, g\}=0$ if $f, g \in \mathcal{O}^{\times}(X), f+g=1$; here \{\} denotes the product in $K^{M}(X)$. By the proposition, the identification $\mathcal{O}^{\times}(X) \stackrel{\sim}{\rightarrow} H_{\mathcal{M}}^{1,1}(X, \mathbb{Z})$ extends to a morphism of graded algebras $K_{a}^{M}(X) \rightarrow H_{\mathcal{M}}^{a, a}(X, \mathbb{Z})$. According to [Ke] (and [NS], lecture 5 of $[\mathrm{MVW}]$, where the case of a field is treated), this is an isomorphism.

6.9. The cdh localization. Below "scheme" means "separated $k$-scheme of finite type".

6.9.1. The cdh topology is the weakest Grothendieck topology on the category of schemes whose covers include every proper or étale map which admits a constructible section (see 4.2.2). Thus every cdh cover admits a constructible section. The cdh topology is stronger than the Nisnevich one.

A proper map $p: Y \rightarrow X$ is called an abstract blow-up if there is an open dense $U \subset X$ such that $p^{-1}(U)_{\text {red }} \rightarrow U_{\text {red }}$ is an isomorphism.

Example. Let $Y \rightarrow X$ be an abstract blow-up and $Z \hookrightarrow X$ be a closed subscheme such that $U:=X \backslash Z$ satisfies the above condition. Then $Y \sqcup Z \rightarrow X$ is a cdh cover. Such cdh covers are called special.

The next lemma ${ }^{39}$ an essential tool:

Lemma. Let $T / X$ be an $X$-scheme proper and generically finite over $X$. Then there exists an abstract blow-up $Y / X$ such that the strict transform $\bar{T}_{\eta_{X}} \subset T \times_{X} Y$ (which is the closure in $T \times_{X} Y$ of the generic fibers $T_{\eta_{X}}$ ) is finite over $Y$.

Proof of Lemma. Since the projection to $X$ of the disjoint union of normalizations of its reduced irreducible components is an abstract blow-up, we can assume that $X$ is integral and normal. By Chow's lemma, we can assume that $T / X$ is projective. Let $U \subset X$ be an open dense subset such that $T_{U} / U$ is finite flat of some degree $d$. We get a section $U \rightarrow \operatorname{Sym}^{d}\left(T_{U} / U\right) \subset \operatorname{Sym}^{d}(T / X)$ (see (a) of the proof in 2.1.3). Our $Y$ is the closure of its image; the promised property holds by (b) in loc. cit.

Below "proper cdh cover" means a proper map which is a cdh cover (i.e., admits a constructible section).

Proposition. (i) Every proper cdh cover $T / X$ can be refined to a composition of special cdh covers $F / X$. Thus the cdh topology is generated by the Nisnevich covers and special cdh covers.

\footnotetext{
${ }^{39}$ This is a special case of [RG] Th. 5.2.2, which says that for any $T / X$ one can find an abstract blow-up $Y / X$ such that the strict transform $\bar{T}_{\eta_{X}}$ is flat over $Y$.
} 
(ii) Every cdh cover $V / X$ admits a refinement $W / X$ which can be factored as $W \rightarrow Q \rightarrow X$ where $W / Q$ is a Nisnevich cover, $Q / X$ is a proper cdh cover.

Proof. (i) We use induction by $\operatorname{dim} X$. Let $Z \subset X$ be a nowhere dense closed subscheme such that $T / X$ admits a section $s$ over $(X \backslash Z)_{\text {red }}$. Let $Y$ be the closure of $s\left((X \backslash Z)_{\text {red }}\right)$ in $T$. Then $Y \sqcup Z / X$ is a special cdh cover. By the induction assumption, we find a composition of special cdh covers $F_{Z} / Z$ which refines the cover $T_{Z}:=T \times_{X} Z$ of $Z$. Then $F:=Y \sqcup F_{Z} \rightarrow X$ is a composition of special cdh covers which factors through $Y \sqcup T_{Z} / X$, hence through $T / X$, q.e.d.

(ii) It suffices to consider the case when $V / X$ is a composition $V \rightarrow U \rightarrow X$ where $U / X$ is a Nisnevich cover, $V / U$ is a proper cdh cover. By (i) we can assume that $V / U$ is an isomorphism at the generic points of $U$; by Chow's lemma, we can assume that $V / U$ is projective.

It suffices to find an abstract blow-up $Y / X$ such that the cover $V \times_{X} Y / Y$ admits a Nisnevich refinement. Indeed, let $Z \subset X$ be a nowhere dense closed subscheme such that $Y \sqcup Z / X$ is a special cdh cover. The cdh cover $\left.V\right|_{Z}$ of $Z$ admits a refinement as in (ii) by induction by $\operatorname{dim} X$, so $V \times_{X}(Y \sqcup Z) / Y \sqcup Z$ admits a refinement $W / Y \sqcup Z$ as in (ii). Then $W / X$ is the promised refinement of $V / X$.

We will find an abstract blow-up $Y / X$ such that each connected component of $Y$ is normal and maps onto an irreducible component of $X$, and the strict transform of $V$ in $V \times_{X} Y$ is finite over $U \times_{X} Y$. Then $V \times_{X} Y / U \times_{X} Y$ admits a (unique) section, ${ }^{40}$ so $V \times_{X} Y / Y$ admits a Nisnevich refinement (namely, $U \times_{X} Y / Y$ ) as needed.

To construct $Y / X$, we compactify $V \rightarrow U \rightarrow X$, i.e., find projective maps $\bar{V} \rightarrow \bar{U} \rightarrow X$ with $U$ an open dense subset of $\bar{U}, \bar{V} \times_{\bar{U}} U=V$, and then apply the lemma to $\bar{V} / X$.

6.9.2. Most applications of the cdh topology require the resolution of singularities, so from now on we assume that char $k=0$. First implications:

(i) For every separated scheme $X$ of finite type there exists a proper cdh cover $\tilde{X} / X$ with smooth $\tilde{X}$. Indeed, there is a special cdh cover $Y \sqcup Z \rightarrow X$ as in Example in 6.9.1 with smooth $Y$, and, by induction by $\operatorname{dim} X$, one can find a proper cdh cover $\tilde{Z} \rightarrow Z$ with $\tilde{Z}$ smooth; set $\tilde{X}:=Y \sqcup \tilde{Z}$.

(ii) Every abstract blow-up $Y \rightarrow X$ of a smooth scheme $X$ is a cdh cover. ${ }^{41}$ Indeed, there exists a sequence of blow-ups $X_{k} \rightarrow \cdots \rightarrow X_{0}=X$ with smooth centers such that the composition factors through $p$, and all $X_{i} \rightarrow X_{i-1}$ are cdh covers for obvious reasons.

(iii) Each proper cdh cover of a smooth $X$ admits a refinement which can be factored as $X_{k} \rightarrow \cdots \rightarrow X_{0}=X$, where $X_{i} / X_{i-1}$ are blow-ups with smooth centers. This follows from (ii), since $Y / X$ admits an abstract blow-up refinement.

Consider the cdh topology on $\mathcal{S} m$. As in 1.11, we have the subcategories

\footnotetext{
${ }^{40}$ For $U \times_{X} Y$ is normal and $V \times_{X} Y \rightarrow U \times_{X} Y$ is an isomorphism over its generic points.

${ }^{41}$ The claim might be false if $X$ is singular: Let $Z \subset \mathbb{P}^{n}$ be any closed subvariety with $Z(k)=\emptyset$; let $X \subset \mathbb{A}^{n+1}$ be the cone over $Z, Y$ be the blow-up of $X$ at 0 . Then $Y / X$ is not a cdh cover.
} 
$I^{\text {cdh }}, I^{\text {cdh } \perp} \subset D \mathcal{P S} h$ of, respectively, cdh locally acyclic and cdh local complexes. We have the following standard objects of $I^{\mathrm{cdh}}$ :

(a) Nisnevich Mayer-Vietoris complexes, see 4.2.1.

(b) Complexes $R[\tilde{Z}] \rightarrow R[\tilde{X}] \oplus R[Z] \rightarrow R[X]$ where $\tilde{X} / X$ is the blow-up with a smooth center $Z \subset X$ and $\tilde{Z} \subset \tilde{X}$ is the preimage of $Z$; the differentials are the difference and the sum of the evident maps.

Proposition. $I^{\mathrm{cdh}}$ is generated by all complexes of types (a), (b).

Proof. As in the proof of the proposition in 4.2.1, we may apply 1.4.2(i), with $S$ the set of standard objects in (a) and (b). Thus it suffices to show that if $F \in I^{\text {cdh }}$ is such that the total complexes $F(X) \rightarrow F(\tilde{X}) \oplus F(Z) \rightarrow F(\tilde{Z})$ for all data of (b) and similar complexes for all data of (a) are acyclic, then $F=0$.

We prove that $F(X)$ is acyclic by induction by $\operatorname{dim} X$. Assuming that $F(Y)$ is acyclic for every $Y$ of dimension $<m$, let us check that for every $X$ of dimension $m$ and $h \in H^{a} F(X)$ there is an open $V \subset X$ with $X \backslash V$ of codimension $\geq n$ such that $\left.h\right|_{V} \in H^{a} F(V)$ vanishes. Since for $n=m+1$ this means that $F(X)$ is acyclic, the claim yields the proposition.

For any $\tilde{X} / X$ as in (b) one has $\operatorname{dim} Z, \operatorname{dim} \tilde{Z}<m$, hence $F(X) \stackrel{\sim}{\rightarrow} F(\tilde{X})$. Thus, by implication (iii) above, every proper cdh cover of $X$ admits a refinement $T / X$, $\operatorname{dim} T=m$, such that $F(U) \stackrel{\sim}{\rightarrow} F\left(T_{U}\right)$ for every open $U \subset X$.

Since $F \in I^{\mathrm{cdh}}$, there is a cdh cover $W / X$ such that $\left.h\right|_{W} \in H^{a} F(W)$ vanishes. By (ii) of the proposition in 6.9.1, we can, refining $W$ if needed, factor it as $W \rightarrow$ $T \stackrel{\pi}{\rightarrow} X$, where $T / X$ is as above, $W / T$ is a Nisnevich cover. By the argument from the proof of the proposition in 4.2.1 (with $X$ from loc. cit. equal to $T$ ), there is an open $V_{T} \subset T$ with $P:=T \backslash V_{T}$ of codimension $\geq n$ such that $\left.h\right|_{V_{T}}=0$. The promised $V$ is $X \backslash \pi(P)$ (here $h_{V}=0$ since $F(V) \stackrel{\sim}{\rightarrow} F\left(T_{V}\right)$ and $T_{V} \subset V_{T}$ ).

6.9.3. Let us show that replacing the Nisnevich topology by the cdh one does not change the category of motives.

Lemma. The cdh coverings define a Grothendieck topology on $R_{\mathrm{tr}}[\mathcal{S} m]$.

Proof. As in the proposition in 4.3, the lemma follows from the next assertion (and implication (i) in 6.9.2): Let $X, Y$ be schemes, $\pi: U \rightarrow X$ be a cdh cover; suppose $Y$ is normal. Then for any $\gamma \in \mathcal{C}$ or $(Y, X)$ there exist a cdh cover $\pi^{\prime}: V \rightarrow$ $Y$ with normal $V$ and $\tilde{\gamma} \in \mathcal{C}$ or $(V, U)$ such that $\pi \tilde{\gamma}=\gamma \pi^{\prime}$.

Since $U / X$ can be refined to a cdh cover which is a composition of Nisnevich covers and proper cdh covers, it is enough to check the claim when $\pi$ is in either of the above classes of covers. For the Nisnevich covers, see the lemma in 4.3.

Suppose $\pi$ is proper. By the argument from the proof of the lemma in 4.3, we can assume that $\gamma$ is given by an irreducible $\Gamma \subset X \times Y$. Let $\eta_{\Gamma}$ be its generic point. By the cdh property, $\eta_{\Gamma} \hookrightarrow X \times Y$ lifts to $\eta_{\Gamma} \hookrightarrow U \times Y$; let $\Gamma^{\prime} \subset U \times Y$ be the closure of its image. Then $\Gamma^{\prime}$ is proper and generically finite over $Y$. By the lemma in 6.9.1 and implication (i) in 6.9.2, there is an abstract blow-up $\pi^{\prime}: V \rightarrow Y$ 
with smooth $V$, such that the strict transform $\tilde{\Gamma}$ of $\Gamma^{\prime}$ is finite over $V$. It yields $\tilde{\gamma} \in \mathcal{C}$ or $(V, U)$ such that $\pi \tilde{\gamma}=\gamma \pi^{\prime}$, q.e.d.

Following 4.4 and 2.3, we define $\mathcal{I}_{\text {tr }}^{\text {cdh }} \subset \mathcal{P}_{\text {tr }}$ as the homotopy idempotent completion of the full pretriangulated subcategory strongly generated by the Nisnevich Mayer-Vietoris complexes and the complexes $R_{\mathrm{tr}}[\tilde{Z}] \rightarrow R_{\mathrm{tr}}[\tilde{X}] \oplus R_{\mathrm{tr}}[Z] \rightarrow R_{\mathrm{tr}}[X]$ for data of type (b) in 6.9.2. The categories $\mathcal{I}_{\mathrm{tr}}^{\Delta \mathrm{cdh}}, I_{\mathrm{tr}}^{\mathrm{cdh}}$, etc., are defined then as in 4.4 replacing index "Nis" by "cdh".

Proposition. (i) One has $\mathcal{I}_{\mathrm{tr}}^{\Delta \mathrm{cdh}}=\mathcal{I}_{\mathrm{tr}}^{\Delta \mathrm{Nis}}$, so $\mathcal{D}_{\mathcal{M}}^{\mathrm{eff}} \stackrel{\sim}{\rightarrow} \mathcal{P}_{\mathrm{tr}} /{ }^{\kappa} \mathcal{I}_{\mathrm{tr}}^{\Delta \mathrm{cdh}}$, etc.

(ii) The subcategories $I_{\mathrm{tr}}^{\Delta}, I_{\mathrm{tr}}^{\mathrm{cdh}} \subset D \mathcal{P S} h_{\mathrm{tr}}$ are compatible (see 1.3).

(iii) For any $\mathbb{A}^{1}$-homotopy invariant complex $F$ of presheaves with transfers one has $H_{\text {Nis }}(X, F) \stackrel{\sim}{\rightarrow} H_{\text {cdh }}^{\cdot}(X, F)$.

Proof. (i) By 6.6 and the proposition in 6.9.2, the generators of $\mathcal{I}_{\mathrm{tr}}^{\mathrm{cdh}}$ lie in $\mathcal{I}_{\mathrm{tr}}^{\Delta \mathrm{Nis}}$, and we are done.

(iii) One has $\mathcal{C}^{\mathrm{Nis}}(F) \in I_{\mathrm{tr}}^{\Delta \mathrm{Nis} \perp}=I_{\mathrm{tr}}^{\Delta \mathrm{cdh} \perp} \subset I_{\mathrm{tr}}^{\mathrm{cdh} \perp}$ (here " $\in$ " is by 4.4(i), "=" by (i) above) and $\operatorname{Cone}\left(F \rightarrow \mathcal{C}^{\mathrm{Nis}}(F)\right) \in I_{\mathrm{tr}}^{\mathrm{Nis}} \subset I_{\mathrm{tr}}^{\mathrm{cdh}}$, so $\mathcal{C}^{\mathrm{Nis}}(F)=\mathcal{C}^{\mathrm{cdh}}(F)$.

(ii) By (iii) and the theorem in 4.5, for $F$ as in (iii) $H_{\text {cdh }}^{\cdot}(X, F)$ is $\mathbb{A}^{1}$-homotopy invariant. Now a cdh variant of the argument in $4.5(\mathrm{a})$ does the job.

Corollary. Let $p: Y \rightarrow X$ be an abstract blow-up, $Z \subset X$ be a closed subscheme such that $p^{-1}(X-Z)_{\mathrm{red}} \stackrel{\sim}{\rightarrow}(X-Z)_{\mathrm{red}}$. Then the motivic localization of the complex $R_{t r}\left[p^{-1}(Z)\right] \rightarrow R_{t r}[Y] \oplus R_{t r}[Z] \rightarrow R_{t r}[X]$ is quasi-isomorphic to 0 .

In turn, the corollary implies that $M(X)$ is a geometric motive (see 2.3) for any (not necessary smooth) scheme $X$.

6.9.4. For a $k$-scheme $X$ we denote by $R_{t r}^{c}[X]$ the presheaf with transfers which assigns to a smooth connected scheme $Y$ the free $R$-module generated by irreducible cycles $\Gamma \subset X \times Y$ such that the projection $p_{1}: \Gamma \rightarrow Y$ is quasi-finite and dominant over $Y$. Notice that $X \mapsto R_{t r}^{c}[X]$ is covariant functorial with respect to proper morphisms and contravariant functorial with respect to flat quasi-finite morphisms. Let $M^{c}(X)$ denote the motivic localization of $R_{t r}^{c}[X]$.

Lemma. For any open $U \subset X$ the motivic localization of the complex

$$
R_{t r}^{c}[X-U] \rightarrow R_{t r}^{c}[X] \rightarrow R_{t r}^{c}[U]
$$

is quasi-isomorphic to 0.

Proof. By the proposition in 6.9.3, it suffices to show that the cdh localization of (6.9.1) is a short exact sequence of cdh sheaves. We only need to check surjectivity of the right arrow. One can assume that $X$ is proper. Take any $\gamma \in R_{t r}^{c}[U](Y)$. We look for a cdh cover $p: V \rightarrow Y$ with smooth $V$ such that $p^{*}(\gamma) \in R_{t r}^{c}[U](V)$ comes from some $\overline{p^{*}(\gamma)} \in R_{t r}^{c}[X](V)$. One can assume that $\gamma$ is given by an irreducible cycle $\Gamma \subset U \times Y$. Let $\bar{\Gamma}$ be its closure in $X \times Y$. Then $\bar{\Gamma} / Y$ is proper and generically finite. By Lemma in 6.9.1 we find an abstract blow-up $p: V \rightarrow Y$ with smooth $V$ such that the strict transform $\bar{\Gamma}_{V}$ of $\bar{\Gamma}$ is finite over $V$. Our $\overline{p^{*}(\gamma)}$ is $\bar{\Gamma}_{V}$. 
For any smooth $X$ of dimension $n$ we define a canonical morphism in $\mathcal{D}_{\mathcal{M}}^{\text {eff tri }}$

$$
\epsilon_{X}^{\prime c}: M^{c}(X) \otimes M(X) \rightarrow R_{\mathcal{M}}(n)[2 n]
$$

as follows. Choose any smooth compactification $X \subset \bar{X}$; set $D=\bar{X} \backslash X$. By the lemma, $M^{c}(X) \stackrel{\sim}{\rightarrow} M(\bar{X}, D):=C^{\mathcal{M}}\left(\left(R_{t r}[\bar{X}] / R_{t r}[D]\right)\right.$. Notice that $D \times X$ is disjoint from $\Delta(X)$ of $X$ in $\bar{X} \times X$. Now (6.9.2) is the composition $M(\bar{X}, D) \otimes$ $M(X)=M(\bar{X} \times X, D \times X) \rightarrow M(\bar{X} \times X, \bar{X} \times X \backslash \Delta(X)) \stackrel{c l(\Delta)}{\longrightarrow} R_{\mathcal{M}}(n)[2 n] ;$ here $\operatorname{cl}(\Delta) \in H_{\mathcal{M}}^{2 n, n}(\bar{X} \times X, \bar{X} \times X \backslash \Delta(X), R)$ is the class of the diagonal cycle, see (6.4.1). It does not depend on the choice of compactification.

Proposition. The pairing $\epsilon_{X}^{c}=\epsilon_{X}^{c}(-n)[-2 n]: M^{c}(X)(-n)[-2 n] \otimes M(X) \rightarrow$ $R_{\mathcal{M}}$ identifies $M^{c}(X)(-n)[-2 n]$ with the dual to $M(X)$.

Proof. We want to check that $\epsilon^{c}$ is non-degenerate, i.e., that the morphism $M^{c} \rightarrow M(X)^{*}(n)[2 n]$ in $\mathcal{D}_{\mathcal{M}}^{\text {tri }}$ it defines (see 6.7.2) is an isomorphism. Let us compute $\epsilon_{X}^{c}$ using a smooth compactification $X \subset \bar{X}$ by a divisor $D$ with normal crossings such that all the irreducible components $D_{i}, i=1, \ldots, k$, are smooth.

Let $F$ be a complex of presheaves with transfers with $F^{>0}=0, F^{0}=R_{\mathrm{tr}}[\bar{X}]$, $F^{-a}=\underset{i_{1}<\ldots<i_{a}}{\oplus} R_{\mathrm{tr}}\left[D_{i_{1} \ldots i_{a}}\right]$ for $a>0$; the differential is the evident one. Here $D_{i_{1} \ldots i_{a}}:=D_{i_{1}} \cap \ldots \cap D_{i_{a}}$. The morphism $F \rightarrow R_{\mathrm{tr}}[\bar{X}] / R_{\mathrm{tr}}[D]$ is a resolution of $R_{\mathrm{tr}}[\bar{X}] / R_{\mathrm{tr}}[D]$.

Let $G$ be a complex of presheaves with transfers with $G^{<0}=0, G^{0}=R_{\mathrm{tr}}[\bar{X}]$, $G^{a}:=\underset{i_{1}<\ldots<i_{a}}{\oplus} R_{\mathrm{tr}}[\bar{X}] / R_{\mathrm{tr}}\left[\bar{X} \backslash D_{i_{1} \ldots i_{a}}\right]$ for $a>0$; the differential is the evident one. The morphism $R_{\mathrm{tr}}[X] \rightarrow G$ is a resolution of $R_{\mathrm{tr}}[X]$ in the category of Nisnevich sheaves.

The evident pairing $R_{\mathrm{tr}}[\bar{X}]^{\otimes 2} \rightarrow R_{\mathrm{tr}}[\bar{X} \times \bar{X}] / R_{\mathrm{tr}}[(\bar{X} \times \bar{X}) \backslash \Delta(\bar{X})]$ extends naturally to $\tilde{\epsilon}_{F G}: F \otimes G \rightarrow R_{\mathrm{tr}}[\bar{X} \times \bar{X}] / R_{\operatorname{tr}}[(\bar{X} \times \bar{X}) \backslash \Delta(\bar{X})]$ whose other nonzero components are $\tilde{\epsilon}_{i_{1} \ldots i_{a}}: R_{\mathrm{tr}}\left[D_{i_{1} \ldots i_{a}}\right] \otimes R_{\mathrm{tr}}[\bar{X}] / R_{\mathrm{tr}}\left[\bar{X} \backslash D_{i_{1} \ldots i_{a}}\right] \rightarrow R_{\mathrm{tr}}[\bar{X} \times$ $\bar{X}] / R_{\mathrm{tr}}[(\bar{X} \times \bar{X}) \backslash \Delta(\bar{X})]$. It also yields a morphism $\tilde{\epsilon}:\left(R_{\mathrm{tr}}[\bar{X}] / R_{\mathrm{tr}}[D]\right) \otimes R_{\mathrm{tr}}[X] \rightarrow$ $R_{\mathrm{tr}}[\bar{X} \times \bar{X}] / R_{\operatorname{tr}}[(\bar{X} \times \bar{X}) \backslash \Delta(\bar{X})]$, so $\tilde{\epsilon}_{F G}$ is a pairing of the resolutions that lifts $\tilde{\epsilon}$.

Let us pass to $\mathcal{D}_{\mathcal{M}}^{\text {eff tri }}$. Composing $\tilde{\epsilon}_{F G}, \tilde{\epsilon}$ with $\operatorname{cl}(\Delta)$, we get $R_{\mathcal{M}}(n)[2 n]$-valued pairings $\epsilon_{F G}, \epsilon$ identified by the resolution quasi-isomorphisms. One has $\epsilon=\epsilon_{X}^{\prime c}$, so it remains to show that $\epsilon_{F G}$ is non-degenerate. It is compatible with the stupid filtrations on $F, G$, so it suffices to check that each $\operatorname{gr}^{a} \epsilon_{F G}$ is non-degenerate. By 6.7.1, this is true for $a=0$, since $\operatorname{gr}^{0} \epsilon_{F G}$ equals $\epsilon_{\bar{X}}^{\prime}$ from (6.7.1). For $a>0$, $\mathrm{gr}^{a} \epsilon_{F G}$ is the direct sum of pairings $\epsilon_{i_{1} \ldots i_{a}}=c l(\Delta) \tilde{\epsilon}_{i_{1} \ldots i_{a}}: M\left(D_{i_{1} \ldots i_{a}}\right) \otimes M(\bar{X}, \bar{X} \backslash$ $\left.D_{i_{1} \ldots i_{a}}\right) \rightarrow R_{\mathcal{M}}(n)[2 n]$. The non-degeneracy comes again from 6.7.1, since the Gysin isomorphism $M\left(\bar{X}, \bar{X} \backslash D_{i_{1} \ldots i_{a}}\right) \stackrel{\sim}{\rightarrow} M\left(D_{i_{1} \ldots i_{a}}\right)(a)[2 a]$ identifies $\epsilon_{i_{1} \ldots i_{a}}$ with $\epsilon_{D_{i_{1} \ldots i} \ldots}^{\prime}$ from (6.7.1), as follows from the next exercise:

Exercise. Let $Z \hookrightarrow Y \hookrightarrow X$ be closed embeddings of smooth varieties of codimensions $n$ and $m$. Then the compositions $M(X, X \backslash Y) \stackrel{\sim}{\rightarrow} M(Y)(n)[2 n] \rightarrow$ $M(Y, Y \backslash Z)(n)[2 n] \stackrel{\sim}{\rightarrow} M(Z)(m+n)[2(m+n)]$ and $M(X, X \backslash Y) \rightarrow M(X, X \backslash Z) \stackrel{\sim}{\rightarrow}$ $M(Z)(m+n)[2(m+n)]$, where $\stackrel{\sim}{\rightarrow}$ are Gysin maps (6.3.1), are homotopic. 
Notation. $\mathcal{A}^{\text {op }} 1.1 ; \mathcal{A}^{\kappa} 1.1,1.5 .1 ; \mathcal{A}^{\text {perf }} 1.4 .2,1.7 ; \mathcal{A}^{\text {pretr }} 1.5 .4 ; \mathcal{A}^{\text {tri }} 1.5 .4 ; \underline{\mathcal{A}}$ 1.6.1; $\mathcal{A}-\bmod 1.1 ; \mathcal{A}-\operatorname{dgm} 1.6 .3 ; \mathcal{A} / \mathcal{I}, \mathcal{A}{ }^{\kappa} \mathcal{I} 1.4 .2,1.8 ; \mathcal{A r} 2.4 ; \operatorname{cl} 6.4 ; C \mathcal{A} 1.5 .1 ;$ $C^{b} \mathcal{A} 1.5 .4 ; \mathcal{C}^{\mathcal{T}} 1.11 ; \mathcal{C}^{\mathcal{M}} 2.3 ; \mathcal{C}^{\Delta} 3.1 ; \mathcal{C}^{\mathrm{Nis}} 4.2 .2 ; \mathcal{C}^{\mathrm{Zar}} 4.1 ; \mathcal{C H}_{\mathcal{M}} 6.7 .3 ; \mathcal{C}$ or, $\mathcal{C}$ or $(\mathcal{S} m)$ $2.1 .2 ; \mathcal{C}{ }^{\Delta}(X, Y), \mathcal{C}^{\Delta}{ }^{\Delta}(\mathcal{S} m) 3.1 .2 ; \mathcal{C} o r(\mathcal{P} a), \mathcal{C}$ or $\left(\mathcal{P} a^{\mathrm{sm}}\right), \mathcal{C}$ or $\left(\left(X^{\prime}, U^{\prime}\right),(X, U)\right)$ $3.4 .1 ; \operatorname{Cor}\left(\mathcal{P} a^{\text {nice }}\right) 5.2 ; \operatorname{Cous}(F) 4.6 .3 ; \operatorname{deg} 2.1 .2 ; \mathcal{D}_{\mathcal{M}}, \mathcal{D}_{\mathcal{M}}^{\text {tri }}, \mathcal{D}_{p \mathcal{M}} 6.1 ; \mathcal{D}_{\mathcal{M}}^{\text {eff }} 2.3 ;$ $\mathcal{D}_{p \mathcal{M}}^{\text {eff }} 3.1 .2 ; F_{-n} 2.2 ; F_{\mathcal{T}} 1.11 ; F_{\text {Zar }} 4.1 .1 ; F_{\mathrm{Nis}} 4.2 .1 ; F_{\text {Nis }}^{\cdot} 4.2 .2 ; H_{\mathcal{M}}^{m, n}(X, R) 6.2 .1 ;$ hocolim 1.4.1; Hom 1.9; Hot $\mathcal{A} 1.5 .1 ; I^{\perp} 1.1 ; i^{\text {Gys }} 6.3 .1 ; I^{\mathcal{T}} 1.11 ; I^{\text {cdh }} 6.9 .2 ; I_{\mathrm{tr}}^{\text {cdh }}$ $6.9 .3 ; \mathcal{I}_{\mathrm{tr}}^{\Delta} 2.3 ; \mathcal{I}_{\mathrm{tr}}^{\mathrm{Nis}} 4.4 ; I_{\mathrm{tr}}^{\mathrm{Nis}} 4.3 ; \mathcal{I}_{\mathrm{tr}}^{\mathrm{Zar}} 2.3 ; K^{M} 6.8 ; M(X) 2.3 ; M^{c}(X) 6.9 .4 ; o$ 2.1.2; $\mathcal{P}, \mathcal{P}_{\text {tr }} 2.1 .4 ; \mathcal{P S} h 1.10,2.1 .1 ; \mathcal{P S} h_{\text {tr }} 2.1 .2 ; \mathcal{S} h_{\mathcal{T}} 1.11, R, R$-mod 1.1; R-dgm 1.6.3; $R_{\mathcal{M}} 2.3 ; R[T], R[U],. R[X / Y] 1.10 ; R(n) 2.2 ; R[G]$-perm $2.4 ; R_{\mathrm{tr}}, R_{\mathrm{tr}}[X] 2.1 .2 ;$ $R_{\mathrm{tr}}(n) 2.2 ; R_{\mathrm{tr}}^{\Delta}[X], R_{\mathrm{tr}}^{\Delta}(n) 3.1 .2 ; R_{\mathrm{tr}}^{c}[X] 6.9 .4 ; \operatorname{Res} 4.6 .2 ; R \Gamma_{\mathcal{M}}(X, R(n)) 6.2 ; \mathcal{S} h^{\mathcal{T}}$ $1.11 ; \mathcal{S} h^{\mathrm{Nis}} 4.2 .1 ; \mathcal{S} h_{\mathrm{tr}}^{\mathrm{Ni}} 4.3 ; \mathcal{S} m 2.1 ; \mathcal{S} m_{0} 2.4 ; S p 3.4 .1 ; \mathrm{Sym} X 2.1 .3 ; \mathbb{T} 3.4 .2 ; X^{(n)}$ 4.6.3; $X^{\mathrm{Nis}} 4.2 .2 ;(X, U) 3.4 .1,(X / S, U) 5.1 .1 \Pi^{\varphi} 6.1 .2 ; \pi_{0} 2.4$.

\section{REFERENCES}

[BS] P. Balmer, M. Schlichting, Idempotent completion of triangulated categories, J. Algebra 236 (2001), no. 2, 819-834.

[BBD] A. Beilinson, J. Bernstein, P. Deligne, Faisceaux pervers, Astérisque 100 (1982).

[BD] A. Beilinson, V. Drinfeld, Chiral algebras, Colloquium Publications, vol. 51, AMS, Providence, RI, 2004.

[BK] A. Bondal, M. Kapranov, Enhanced triangulated categories, Math. USSR-Sbornik 70 (1991), no. 1, 93-107.

[Bo1] M. Bondarko, Differential graded motives: weight complex, weight filtrations, and spectral sequences for realizations; Voevodsky vs. Hanamura, math. AG/0601713 (2006).

[Bo2] M. Bondarko, Weight structures for triangulated categories: weight filtrations, weight spectral sequences and weight complexes; applications to motives and to the stable homotopy category, math. KT/0704.4003 (2007).

[BG] K. Brown, S. Gersten, Algebraic K-theory as generalized sheaf cohomology, Algebraic K-theory, I, Lect. Notes in Math., vol. 341, Spinger, Berlin-Heidelberg-New York, 1973, pp. 266-292.

[Deg1] F. Déglise, Around the Gysin triangle, http://www.math.uiuc.edu/K-theory/764 (2006).

[Deg2] F. Déglise, Finite correspondences and transfers over a regular base, http://www.math. uiuc.edu/K-theory/765 (2006).

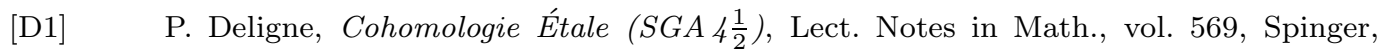
Berlin-Heidelberg-New York, 1977.

[D2] P. Deligne, Catégories tannakiennes, The Grothendieck Festschrift, Vol. II, Progr. Math., vol. 87, Birkhuser, Boston, MA, 1990, pp. 111-195.

[Dr] V. Drinfeld, DG quotients of DG categories, J. Algebra 272 (2004), no. 2, 643-691.

[EGA II] A. Grothendieck, J. Dieudonné, Éléments de géométrie algébrique, Ch. II, Publ. Math. IHES 8 (1961).

[EGA IV] A. Grothendieck, J. Dieudonné, Éléments de géométrie algébrique, Ch. IV, Publ. Math. IHES 28 (1966).

[F] W. Fulton, Intersection theory, Ergebnisse der Mathematik und ihrer Grenzgebiete, 3 Folge, Springer-Verlag, Berlin, 1998.

[G] O. Gabber, Gersten's conjecture for some complexes of vanishing cycles, Manuscripta 
Math. 85 (1994), no. 3-4, 323-343.

[Go] R. Godement, Théorie des faisceaux, Hermann, Paris, 1958.

[K] B. Keller, Deriving DG categories, Ann. Sci. ÉNS, 4 série, 27 (1994), 63-102.

[Ke] M. Kerz, The Gersten conjecture for Milnor K-theory, http://www.math.uiuc.edu/Ktheory/0791 (2006).

[L1] J. Lurie, Higher topos theory, math. CT/0608040 (2006).

[L2] J. Lurie, Derived algebraic geometry, math. CT/0608228, 0702299, 0703204,...

[M] C. Mazza, Schur functors and motives, K-Theory 33 (2004), no. 2, 89-106.

[MVW] C. Mazza, V. Voevodsky, C. Weibel, Lectures notes on motivic cohomology, Clay Mathematics Monographs, vol. 2, AMS, Providence, RI, 2006.

[N] A. Neeman, The connection between the K-theory localization theorem of Thomason, Trobaugh and Yao and the smashing subcategories of Bousfield and Ravenel, Ann. Sci. ENS 25 (1992), no. 5, 547-566.

[NS] Yu. Nesterenko, A. Suslin, Homology of the general linear group over a local ring, and Milnor's K-theory, Math. USSR-Izv. 34 (1990), no. 1, 121-145.

[Ni] Y. A. Nisnevich, The completely decomposed topology on schemes and associated descent spectral sequences in algebraic K-theory, Algebraic K-theory: connections with geometry and topology (Lake Loise, AB, 1987), Kluwr Acad. Publ., Dordrecht, 1989, pp. 241-342.

[RG] M. Raynaud, L. Gruson, Critères de platitude et de projectivité. Techniques de "platification" d'un module, Invent. Math. 13 (1971), 1-89.

[R] R. Rouquier, A letter from 10.21.06.

[SGA4] Théorie de Topos et Cohomologie Étale des Schémas. Séminaire de Géométrie Algébrique du Bois-Marie 1963-1964. Dirigé par M. Artin, A. Grothendieck, J. L. Verdier, Lect. Notes in Math., vol. 269, 270, 305, Spinger, Berlin-Heidelberg-New York, 1972-1973.

[S] A. Suslin, A talk at K-theory conference, Luminy (1987).

[SV] A. Suslin, A. Voevodsky, Singular homology of abstract algebraic varieties, Invent. Math. 123 (1996), no. 1, 61-94.

[T] D. Tamarkin, What do dg-categories form?, Compositio Math. 143 (2007), no. 5, $1335-1358$.

[Th] R. Thomason, The classification of triangulated subcategories, Compositio Math. 105 (1997), no. 1, 1-27.

[TT] R. Thomason, T. Trobaugh, Higher algebraic K-theory of schemes and of derived categories, The Grothendieck Festschrift, Vol. III, Progr. Math., vol. 88, Birkhuser, Boston, MA, 1990, pp. 247-435.

[Ve] J.-L. Verdier, Des catégories dérivées des catégories abéliennes, Astérisque 239 (1996).

[Vo1] V. Voevodsky, Cohomological theory of presheaves with transfer, Cycles, transfers, and motivic homology theories, Annals of Mathematics Studies, vol. 143, Princeton University Press, Princeton, NJ, 2000, pp. 87-137.

[Vo2] V. Voevodsky, Triangulated categories of motives over a field, Cycles, transfers, and motivic homology theories, Annals of Mathematics Studies, vol. 143, Princeton University Press, Princeton, NJ, 2000, pp. 188-238.

[Vo3] V. Voevodsky, Cancellation theorem, http://www.math.uiuc.edu/K-theory/541 (2002).

[Vo4] V. Voevodsky, Homotopy theory of simplicial sheaves in completely decomposable topolo- 
gies, http://www.math.uiuc.edu/K-theory/443 (2000). 